\title{
ADAPTIVE CONTROL BASED ON ORTHONORMAL SERIES REPRESENTATION
}

By

Christos Constantine Zervos

B. Sc. University of Athens, Athens, 1981

M. Eng. McGill University, Montreal, 1984

A THESIS SUBMITTED IN PARTIAL FULFILLMENT OF

THE REQUIREMENTS FOR THE DEGREE OF

DOCTOR OF PHILOSOPHY

in

THE FACULTY OF GRADUATE STUDIES

DEPARTMENT OF ELECTRICAL ENGINEERING

We accept this thesis as conforming

to the required standard

THE UNIVERSITY OF BRITISH COLUMBIA

March 1988

(c) Christos Constantine Zervos 
In presenting this thesis in partial fulfilment of the requirements for an advanced degree at the University of British Columbia, I agree that the Library shall make it freely available for reference and study. I further agree that permission for extensive copying of this thesis for scholarly purposes may be granted by the head of my department or by his or her representatives. It is understood that copying or publication of this thesis for financial gain shall not be allowed without my written permission.

Department of ELECTRICAL ENGINEERING

The University of British Columbia

Vancouver, Canada

Date OCTOBER 26, 1988 


\begin{abstract}
This thesis describes a novel approach to adaptive control systems design using orthonormal series representation. The class of adaptive algorithms considered is that commonly referred to as self-tuning controllers developed for discrete-time systems. A common characteristic of the self-tuning schemes so far studied for industrial applications is that they are usually based on ARMAX models. These existing adaptive control algorithms have been shown to be globally asymptotically stable under certain theoretical assumptions and they have performed well in various applications. These theoretical assumptions are somehow too restrictive from an engineering and practical point of view. Real industrial plants always contain considerable time delays, have unmodeled dynamics, exhibit time varying dynamics and are subject to various disturbances.
\end{abstract}

The purpose of this thesis is to explore a new way of representing and controlling dynamic systems in an effort to find another way, probably better and more robust, to handle a certain class of industrial applications. The behaviour of adaptive controllers in the presence of unmodelled dynamics and in the presence of time-varying plant delays along with the need for reduced a-priori information as dictated by the conditions encountered usually in practice have led us to abandon the usual ARMA transfer function representation for a new representation by orthonormal series. Our new approach is advantageous because it eliminates the need for assumptions about the plant order and the time-delay, i.e. accurate assumptions about their true values are not necessary. A physical dynamical plant, including its time delay, is modelled by an orthonormal set of functions. The sets considered here are mainly the Laguerre set, a modified version 
of it, and a set based on complex poles. Other orthonormal functions may also be used. A simple predictive control law is proposed from which an adaptive controller is then designed. The schemes developed are explicit and implicit, deterministic and stochastic. Some multivariable schemes are also presented. Simulations of these new controllers show they are easy to use, able to handle non-minimum phase plants, and more robust than the conventional model-based approaches. Results from industrial trials confirm the applicability of these new schemes. 
Table of Contents

Abstract $\quad$ ii

List of Tables $\quad \cdots \quad$ vii

List of Figures $\quad$ xii

Acknowledgement $\quad$ xiii

1 Introduction 1

1.1 General Introduction . . . . . . . . . . . . . . . . . 1

1.2 Brief summary of previous work . . . . . . . . . . . . 4

1.3 Contribution of this thesis $\ldots \ldots \ldots \ldots \ldots \ldots \ldots$

1.4 Outline of the Thesis $\ldots \ldots \ldots \ldots \ldots \ldots \ldots$

2 Laguerre Functions in Modeling and Identification $\quad 14$

2.1 Signal Description by Orthonormal Functions . . . . . . . . . . . 14

2.2 The Laguerre functions $\ldots \ldots \ldots \ldots \ldots \ldots$

2.2.1 Introducing the Laguerre orthonormal set . . . . . . . . . 18

2.2.2 Least Squares Estimation of the Laguerre gains . . . . . . . . 22

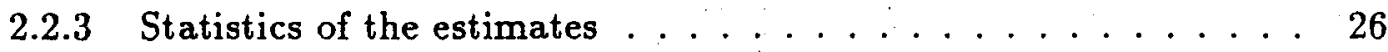

2.2.4 The Discrete-time Laguerre set . . . . . . . . . . . . 31

2.3 Modelling of dynamic systems . . . . . . . . . . . . . 35

3 Deterministic Adaptive Control 41 
3.1 The Predictive Control Law . . . . . . . . . . . . . 41

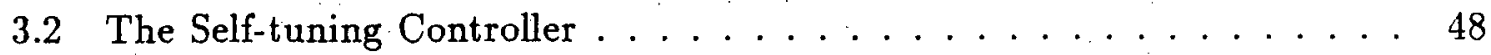

3.2.1 A Deterministic Explicit Self-Tuner . . . . . . . . . . . . 48

3.2.2 A Deterministic Implicit Self-tuner . . . . . . . . . . . . . 53

3.3 Simulation results $\ldots \ldots \ldots \ldots \ldots \ldots \ldots \ldots \ldots \ldots \ldots$

3.4 Robustness issues . . . . . . . . . . . . . . . . . . 64

3.5 Practical Aspects and Implementation $\ldots \ldots \ldots \ldots \ldots$

3.5.1 Choice of Laguerre filter time constant . . . . . . . . . 73

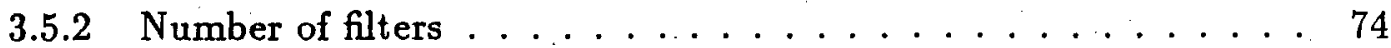

3.5.3 Choice of $d$ and $\alpha \ldots \ldots \ldots \ldots \ldots \ldots \ldots \ldots$

4 Stochastic Adaptive Control $\quad 76$

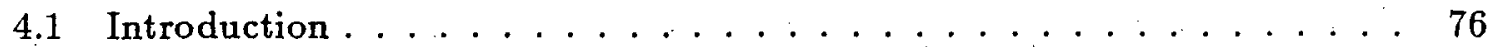

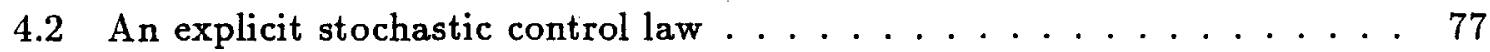

4.2 .1 Stability analysis $\ldots \ldots \ldots \ldots \ldots \ldots \ldots$

4.2 .2 Practical Aspects and Simulation . . . . . . . . 85

5 Multivariable Self-Tuning Control

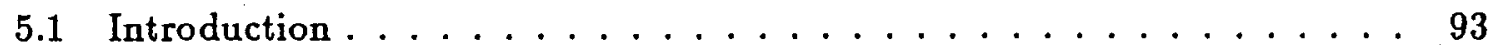

5.2 Deterministic Multivariable Self-Tuning Control . . . . . . . . . . . . 94

5.3 Stochastic Multivariable Self-Tuning Control _ . . . . . . . . . 99

5.4 Simulation examples . . . . . . . . . . . . . . . . 102

6 An Industrial Application $\quad 110$

6.1 Introduction . . . . . . . . . . . . . . . 110

6.2 Laguerre-based adaptive control of $\mathrm{pH}$ in an industrial bleach plant extraction stage 
7 Using other orthonormal sets

7.1 A modified Laguerre set . . . . . . . . . . . . . . . . 124

7.2 A set with discrete complex exponentials . . . . . . . . . 127

8 Conclusions

Bibliography 


\section{List of Tables}

2.1 The values of the 12 identified Laguerre gains for each case. . . . . . 39

4.2 Example 4.1: The choice of the various parameters during the simulation

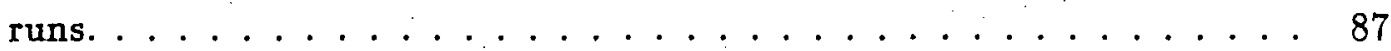

6.3 The 15 Laguerre gains as recorded at September $4,20: 45 \ldots \ldots \ldots 117$ 


\section{List of Figures}

2.1 Approximation of a transfer function by a linear combination of or-

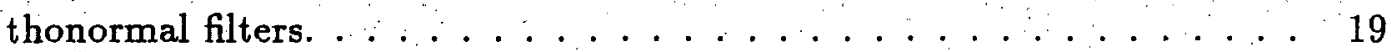

2.2 Laguerre Ladder Network. $\ldots \ldots \ldots \ldots \ldots \ldots$

2.3 The Continuous Network Compensation of $G^{*}(s) \ldots \ldots \ldots 33$

2.4 Impulse response of a triangle-hold $G_{h}(s) \ldots \ldots \ldots \ldots$

2.5. Example 2.1 : Unit step responses of the true plant and the identified one for the case without output noise (The 2 curves are identical). $\ldots 39$

2.6 Example 2.1 : Unit step responses of the true plant (3), the identified one when $\sigma=0.5(2)$, and when $\sigma=0.8(1) \ldots \ldots \ldots \ldots 40$

3.7 System to be controlled in Example 3.1. . . . . . . . . . . . 54

3.8 Example 3.1: Output responses of $H\left(q^{-1}\right)$, (a) with and (b) without noise $k_{d}=1 . \ldots \ldots \ldots \ldots \ldots \ldots \ldots \ldots \ldots \ldots \ldots \ldots \ldots \ldots \ldots \ldots$

3.9 Example 3.1: Output responses, (a) with and (b) without noise, when $H\left(q^{-1}\right)$ is under Laguerre self-tuning control, $k_{d}=1, d=2 . \quad \ldots \ldots 56$

3.10 Example 3.1: Output responses, (a) with and (b) without noise, when $H\left(q^{-1}\right)$ is under Laguerre self-tuning control, $k_{d}=3, d=4 \quad \ldots \ldots . \quad 57$

3.11 Example 3.2: System response under Laguerre self-tuning control, $1^{\text {st }}$

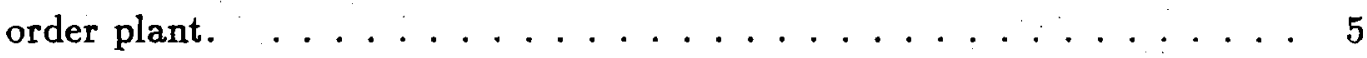

3.12 Example 3.2: System response under Laguerre self-tuning control, $2^{\text {nd }}$

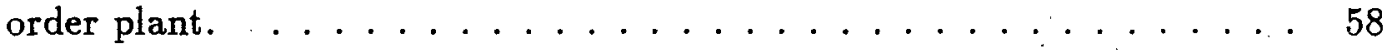

3.13 Example 3.3: Laguerre self-tuning Control of a system with integrator. $\quad 60$ 
3.14 Example 3.4: Manipulator Arm tracking a circular trajectory. Payload

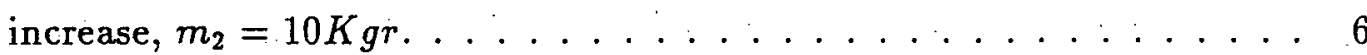

3.15 Example 3.4: Manipulator Arm tracking a step trajectory. Response of $2^{\text {nd }}$ joint angle. Inductance increase. $d=4 \ldots \ldots \ldots \ldots \ldots$

3.16 Example 3.4: Manipulator Arm tracking a step trajectory: Response of $1^{s t}$ joint angle. Inductance increase. $d=12 \ldots \ldots \ldots \ldots$

3.17 Example 3.5: Plant output following a square-wave setpoint when an

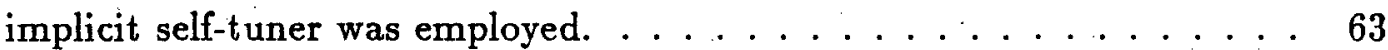

3.18 Example 3.5: Controller's output for the implicit self-tuning scheme. . . 64

3.19 Example 3.6: Output tracking the reference input when $N=1, T=$

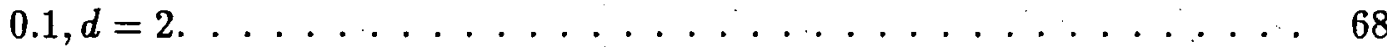

3.20 Example 3.6: Bode plots for the true (solid lines) and identified (dash lines) systems, when $N=1, T=0.1, d=2 \ldots \ldots \ldots \ldots \ldots$

3.21 Example 3.6: System output tracking the reference input when $N=$ $1, T=0.01, d=10$. (The 2 curves are almost identical) $\quad \ldots \ldots 69$

3.22 Example 3.6: (a) System output tracking the reference input when $N=$ $2, T=0.01, d=2$. (b) An enlargment portion from $T=20$ to 23 . (The 2 curves are almost identical.) $\ldots \ldots \ldots \ldots \ldots \ldots \ldots$

3.23 Example 3.6: Bode plots for true (solid lines) and identified (dash lines) systems, when $N=2, T=0.01, d=2$.

3.24 Example 3.7: System output tracking the reference input (above) and controller output (below). $(N=16, p=0.8, d=10, T=1.0$. At the $800^{\text {th }}$ point we switch to a different plant) . . . . . . . .

4.25 Orthonormal Modelling of a stochastic system . . . . . . . . . . 78 
4.26 Example 4.1: Explicit stochastic self-tuner. System output tracking a square wave set-point. Case 1 of Table $4.2 \ldots \ldots . \ldots 8$

4.27 Example 4.1: Explicit stochastic self-tuner. System output tracking a square wave set-point. Case 2 of Table 4.2. . . . . . . . . . . . . 88

4.28 Example 4.1: Explicit stochastic self-tuner. System output tracking a square wave set-point. Case 3 of Table 4.2. . . . . . . . . . . . . 89

4.29 Example 4.1: Explicit stochastic self-tuner. System output tracking a square wave set-point. Case 4 of Table $4.2 \ldots \ldots$. . . . . . . . . 89

4.30 Example 4:1: Explicit stochastic self-tuner. System output tracking a square wave set-point: Case 5 of Table 4.2. . . . . . . . . . . . 90

4.31 Example 4.1: Explicit stochastic self-tuner. System output tracking a square wave set-point. Case 6 of Table $4.2 \ldots \ldots \ldots$. . . . . . . . . 90

4.32 Example 4.1: Explicit stochastic self-tuner. System output tracking a square wave set-point. Case 7 of Table $4.2 \ldots \ldots \ldots 1$

4.33 Example 4.1: Explicit stochastic self-tuner. System output tracking a square wave set-point. Case 8 of Table $4.2 \ldots \ldots \ldots$. . . . . . 91

4.34 Example 4.1: Implicit stochastic self-tuner. System output tracking a square wave set-point. ................... 92

5.35 Multivariable $2 \times 2$ input-output linear system. . . . . . . . . . 94

5.36 Laguerre Ladder Network for a Multivariable $2 \times 2$ input-output linear

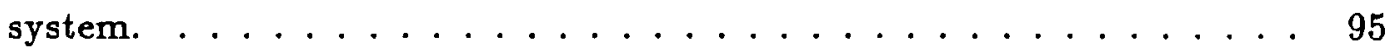

5.37 Stochastic multivariable $2 \times 2$ linear system. . . . . . . . . . . 99

5.38 Example 5.1: Expl: cit multivariable self-tuning control of a $2 \times 2$ plant. System outputs tracking two respective reference trajectories. . . . . . 103 
5.39 Example 5.1: Explicit multivariable self-tuning control of a $2 \times 2$ plant.

Controller outputs. . . . . . . . . . . . . . . . . . 104

5.40 Example 5.2: Headbox of a paper-machine. . . . . . . . . . 105

5.41 Example 5.2: Headbox multivariable self-tuning control. Stock level (upper curve), and Total head pressure (lower) changes. . . . . . 109

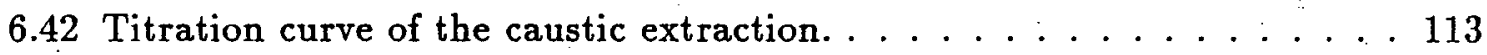

6.43 Bleach Plant. $1^{\text {st }}$ Alkaline Extraction stage. . . . . . . . . . . . . 114

6.44 Block diagram of $1^{\text {st }}$ Alkaline extraction stage. . . . . . . . . 115

$6.45 p H$ loop. Alkaline extraction stage. Open-loop step response. . . . . 115

6.46 Tower exit $p H$ with the outer loop always under PID control. $3 \mathrm{~min} / \mathrm{sample-}$

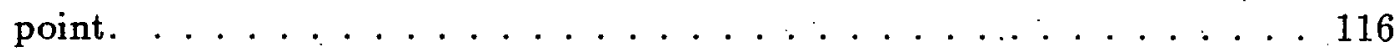

$6.47 p H$ loop. Comparison of regulation performances. $3 \mathrm{~min} /$ sample-point. . 119

6.48 Step response of identified $p H$-loop. $8 \mathrm{~min} /$ sample-point. . . . . . 120

6.49 Self-tuner output in $p H$ values. $3 \mathrm{~min} /$ sample-point. . . . . . . . 120

6.50 Alkaline extraction stage. Caustic flow. $3 \mathrm{~min} /$ sample-point. . . . . . . 121

6.51 Alkaline extraction stage. J-Tube exit $p H .3 \mathrm{~min} /$ sample-point. . . . . 121

6.52 Alkaline extraction stage. Performance over the period 28-30 November,

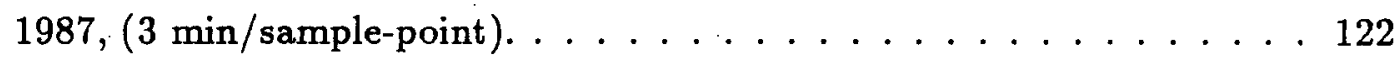

6.53 Autocorrelation comparisons between the PID/Manual and Self-tuner

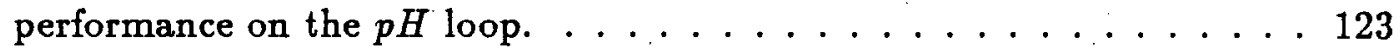

7.54 Modified Laguerre Ladder Network. . . . . . . . . . . . . . . . 125

7.55 Ladder Network for the orthonormal set of functions with complex poles. 131

7.56 Example 7.1: Step-response comparison of the true plant (solid) and the identified one (dash line) using the Laguerre set $(N=12) \ldots \ldots \ldots 134$ 
7.57 Example 7.1: Step-response comparison of the true plant.(solid) and the identified one (dash line) using the Complex set $(N=6) \ldots \ldots \ldots 134$

7.58 Example 7.1: Bode-plot comparison of the true plant (solid) and the identified one (dash line) using the Complex set $(N=6) \ldots \ldots \ldots 135$ 


\section{Acknowledgement}

A Ph.D. thesis represents not only a piece of technical research work but among other things it is to be considered as a positive contribution to knowledge representing one of the first phases of one's professional life. This achievement would not have been possible without the contribution of many other motivational factors. Thus I take this time to acknowledge and thank not only those who aided with the research itself and those who aided me personally through the years of research, but also those who brought me to point where I was prepared to undertake the task of earning a doctorate.

Among the technical contributors I especially thank Prof. Guy Dumont my thesis advisor, for guiding my progress throughout the research, for his interest and enthusiasm about the subject, for his continuous supporting help and guidance and for his belief and trust in me. I thank Dr. Pertti Makila and Dr. K. Natarajan while they stayed as post-doctoral fellows with the UBC Pulp and Paper Centre, for their time in discussing any topic of interest, including many details of this thesis. They also proved to be invaluable friends and their advice and concern was a big motivational factor for

me. I especially also thank Mr. Darrell Wong for performing the simulation runs for the manipulator arm example presented in Chapter 3 of this thesis. Thanks also to Dr. C.J. Bennington for providing the data for the Figure 6.42.

Foremost among those who brought me to the point where a $\mathrm{Ph} . \mathrm{D}$ was in the realm of possibility are my parents, Constantine and Alice Zervos. The knowledge they imparted through the loving attention I received during my stay in their "old-fashioned" home and through the interest and loving care they showed me during all my student years in Canada are ones of the most valuable experiences that I have ever had in my 
life. I also thank my sisters, Stella, Lila and her husband George and my lovely niece Katerina for their immesurable love and care they always showed me especially during the hard times.

I thank all the teachers I have had through all of these twenty-four years of formal education, especially Prof. Evangelos Servetas, my supervisor while I stayed at the Nuclear Research Centre, "Demokritos", Athens , Greece, for he was the one who initiated me in the field of Control Engineering and paved my entrance to the way that led me to earn these higher graduate studies. I also very much thank, Prof. Pierre Belangér my M.Eng. thesis advisor from McGill University for all his help, support, interest, new ideas, enthousiasm and especially his guidance. My greatest thanks go to Prof. Guy A. Dumont from the Pulp and Paper Research Institute of Canada, and his wonderful loving family. His friendly attitude, technical expertise, academic knowledge, cooperative manners, thoughtful consideration and their advice and assistance proved invaluable to me and strengthened my courage to continue and complete my studies.

I would also like to thank all the professors and students from the Pulp and Paper Centre and from the Electrical Engineering Department of UBC. I thank Ed Casas, Jim Reimer, Alan Kot, for their valuable friendship and the constructive times we had together during my stay at UBC. I would like also to thank all my friends who have helped me with one way or another during all these schooling years. Near their loving support I found all these exceptional nice qualities that make this world a better place to live. I thank the Dumonts, the Davies, the Terezakis, the Khayats, and all who have touched my life years in Vancouver, Montreal and Athens and helped to make them good.

The research of this thesis was carried out at the Electrical Engineering Department and at the Pulp and Paper Centre with support provided by the Pulp and Paper Research Institute of Canada under an F.L. Mitchel memorial scholarship and by the 
N.S.E.R.C. under grant $N o$ A-5960. 


\section{Chapter 1}

\section{Introduction}

\subsection{General Introduction}

Over the last two decades, there has been an extensive interest in feedback control systems which automatically adjust their controller settings to compensate for changes in the process or the environment. Numerous adaptive control schemes have been developed and tested with various degrees of success. The number of applications, though small in relation to the activity in the field, is now sufficient to give credibility to adaptive control techniques. Adaptive controllers have become popular despite the fact of their non-linear structure because of their inherent practical design philosophy that renders them as a solution to the problem of tuning industrial controllers. They have considerable potential for process control problems since they provide a systematic, flexible approach for controlling processes which are not well understood or which have significant non-linearities or time-varying parameters. A class of these adaptive control systems, introduced as an approximation of the general non-linear stochastic problem, has the capability to provide good control. They are generally known as self-tuning regulators (Kalman, 1958 ; Aström and Wittenmark, 1973; Clarke and Gawthrop, 1975). Research on adaptive control has emphasized two general approaches: Self-Tuning controllers, and Model Reference adaptive controllers (MRAC), (Landau,1974). Despite their apparently different formulation, objectives, and origins, the two approaches are closely related (Egardt, 1980 ; Landau, 1978). 
In the MRAC approach, the design addresses the problem of self-adjusting the parameters of a controller in order to stabilize the dynamic characteristics of a negative feedback control system while the closed-loop system follows a pre-determined reference model. MRAC can be traced back to the well-known "MIT rule" (Whitaker et al, 1958). Parks in 1966 introduced the stability theory concepts into the design procedures. The multivariable case was then treated by Landau in 1973. Other model reference adaptive algorithms were proposed for non-minimum phase systems by Ionescu and Monopoli (1977) and Narendra et al (1980) with some results on convergence and stability analysis under stringent conditions.

The self-tuning control approach has been proposed mainly as a means for tuning digital controllers for industrial processes. In this case an adaptive algorithm can first be used for tuning the controller parameters, and then be removed after the parameters have converged to proper values. The method of designing these regulators is generally by first performing an identification experiment to get a model of the process and its disturbances and then determining an optimal controller to satisfy a certain performance criterion. At each sampling instant, a dynamic model of the process is updated by estimating the model parameters from input-output data and then the controller parameters are updated based on the newly-obtained model parameters and on a predefined optimization objective. The scheme is either called Indirect if the identification gives a model of the process itself or, Direct if the controller's parameters are directly identified. Two basic assumptions are usually made to simplify the regulator design : (a) the Certainty Equivalence principle where the unknown true parameter values are replaced by the estimated ones and (b) the Separation principle i.e. the separation of the estimation of the process parameters and the computation of the control signal (Wittenmark, 1975). A common characteristic for the schemes so far is that they are model-based. In particular, for the input-output case, the discrete polynomial 
ARMAX (Autoregressive-Moving Average-Exogenous) model representation for the dynamics plus noise transfer functions has been widely used as the main mathematical tool in designing, studying, and analysing these regulators (Åström, 1983).

Various identification methods (e.g. Least Squares, Maximum Likelihood, Instrumental Variables, Stochastic approximation, etc.) have been developed for building mathematical models of dynamic systems based on observed data (Åström and Eykhoff, 1971). A vast variety of control laws (e.g Minimum Variance, Pole/Zero Placement, Linear Quadratic Gaussian, Model Reference, Predictive Control, etc.) has been proposed for implementing the necessary feedback to control succesfully a dynamic system and realize a self-tuning scheme. A thorough review of various self-tuning schemes is given by Goodwin and Sin (1984). A common thread to this work is the use of transfer function models. If the actual plant can be described by the structure of the model, these schemes behave well. However, when this is not the case, performance degradation with potential destabilization occurs (Rohrs et al., 1985). Current adaptive control schemes deal well with structured uncertainty, but cannot adequately handle. unstructured uncertainty. This explains why the behaviour of adaptive controllers in the presence of unmodelled dynamics has been a topic of concern in the adaptive control community in recent years. In summary, typical problems arising in the design of a self-tuning scheme are: (a) the robust behaviour of the algorithm in presence of unmodelled dynamics, (b) the rate of convergence, (c) the stability of the closed- loop system, (d) the reduction of the need for exact a-priori information about the plant e.g. the order, the time delay, the phase characteristics, the number of poles and zeroes, and (e) the numerical properties of the algorithm as they appear in the actual implementation and its capability to deal with a variety of linear or non-linear plants.

The problem of controlling an unknown, non-linear, slowly-time-varying process subject to stochastic disturbances is a common situation in the process industry. The 
limitations often imposed by the lack of knowledge about the plant and its stochastic environment present another major difficulty in the problem formulation. A robust adaptive control scheme requiring minimal a-priori information and capable of producing satisfactory control is a very desirable and attractive solution to the problem. Since the self-tuning control idea is based on a procedure for on-line estimation of a model for the plant dynamics before any control is applied it is imperative that this identification procedure is really robust.

Our search for a robust adaptive control requiring minimal a priori information has led us to the development of unstructured adaptive control. We abandoned the usual ARMAX model for an orthonormal series representation of the plant dynamics. The major advantage of this approach is that any stable plant can be modelled without structural knowledge, i.e. without assumption about the true plant order and time delay. The approach of using orthonormal functions to build models for systems and plants is not new. However, the approach of using these functions to simultaneously model and control plants in a self-tuning scheme is completely new and has never been reported elsewhere.

\subsection{Brief summary of previous work}

Adaptive control has been a great challenge to control engineers for a long time and it has raised an abundant interest in the recent years equally among mathematicians and engineers. Many schemes have been proposed, some of which have found their way succesfully into industrial practice ( $\AA$ ström, 1980b, 1983 ; Goodwin and Sin, 1984). There are also products on the market such as Electromax V from Leeds and Northrup (Hoopes et al, 1983), the Novatune from ASEA (Bengtsson and Egardt, 1984), the Autotuner from NAF Controls, the Exact from Foxboro, and few others. 
Adaptive control has certain advantages (Goodwin and Sin , 1984) when compared with other classical control techniques and methods:

- Adaptive control diminishes system sensitivity.

- Systems with unknown parameters can be controlled.

- The effects of changes in the dynamics can be corrected in real time.

- Simplicity in structure and design.

- Numerical requirements are not that demanding.

- Attractive solution for automatic tuning of process control loops.

The present interest in self-tuning controllers was initiated by the work of $\AA$ ström, and Wittenmark, (1973), who applied recursive least squares and a minimum variance strategy to obtain a self-tuning minimum variance regulator. Similar approaches had previously been proposed by Kalman (1958) and Peterka (1970). A more general approach was presented by Clarke and Gawthrop (1975) to include control of stable nonminimum phase systems by using a generalized output function and employing a cost function which included a penalty on the control input. Similar self-tuning algorithms were developed for pole-zero placement control schemes (Wellstead, Edmunds, Prager, Zanker, 1979 ; Åström and Wittenmark, 1980; Aström, 1980a; Elliot, 1982). The use of a long range predictor in adaptive control was first considered by Ydstie (1982) for SISO systems known as Extended Horizon Predictive Control, (Ydstie 1984). A similar predictive adaptive control scheme known as APCS (Adaptive Predictive Control System) had also been studied by Martin-Sanchez (1976). The Generalized Predictive Control (GPC) strategy introduced by Clarke, Mohtadi and Tuffs (1987) is based on 
the minimization of a finite-time horizon quadratic cost function of the future errors and control increments.

To overcome the problem that a precise knowledge of the model structure was required a-priori in the implementation of the control system many authors (Akaike, 1974; Rissanen, 1979) derived criteria which take model complexity into account in order to obtain a parsimonious model. The Model Predictive Heuristic Control adaptive strategy proposed by Richalet, Rault, Testud, and Papon (1978) relied on the impulse response representation of the process, which constituted the internal model. A predictive control scheme was used for control. Because of the non-structural model approach of that scheme it gained considerable success in many industrial applications. Although the scheme was initially non-adaptive the concepts are easily carried over to the self-tuning approach. An innovative approach of a self-tuning controller utilizing unity feedback and an internal model of the exogenous signals ensuring stability, asymptotic tracking and regulation in the presence of finite parameter perturbations, has been reported by Song, Shah and Fisher (1986). The controller has an error-driven robust structure (Francis and Wonham, 1975; Davison,1976). Recently, self-tuning controllers based on a generalized $H_{\infty}$ control law to guarantee asymptotic stability for stable, unstable, minimum and non-minimum-phase plants have been reported by Grimble (1987).

As the theory underlying the single-input, single-output (SISO) self-tuning control has been well studied during the last decade, attention has turned toward extension of these ideas to more complex situations where effects of disturbances, nonlinearities and unmodeled dynamics are considered. The already published literature on the implicit and explicit algorithms of stochastic adaptive control is vast. Many algorithms have been described in the literature ( $\AA$ ström, 1981) for the adaptive control of linear systems (for a survey see, Seborg et al, 1986). Algorithms that combine various on-line 
identification techniques and different controller design schemes. Of these algorithms, those based on minimum variance control have received particular attention. This has probably been a result of two main factors : the relative ease of implementing these algorithms and the availability of substantial supporting theory. MRAC and self tuning schemes have been found to work well when parameters of the model structure are known, but fail to give stable control performance when high frequency modelling errors or perturbations are present (Rohrs, 1982 ; Rohrs et al, 1982).

Most adaptive controllers are based on a separation between the estimation of the unknown parameters and state variables, and the determination of the control signal. This means that the control laws have not been designed to facilitate the identification. As a solution to this problem dual adaptive controllers emerged. In a dual controller there is an interaction between identification and control in the sense that the controller must compromise between a control action and a probing action (Feldbaum 1960,1961). Although this approach is very attractive and necessary to assure that the controller will not give "turn off" of the control signal, the solution leads to a functional equation which in most cases is difficult to solve.

As a continuation of the SISO stochastic adaptive control system design, significant progress has been made to extend the SISO design to the multi-input, multi-output (MIMO) case. The extension of the algorithms to the MIMO case rests upon the determination of an appropriate generalisation of the notion of the delay and the noncommutativity of the various polynomial matrices involved (Goodwin and Sin, 1984). Along these lines a number of different schemes have been developed. MIMO control schemes have been investigated for a long time. The first ones were based on frequency response analysis and classical control theory. Early workers on the field were more interested of achieving decoupling among the inputs and the outputs of the system by applying decoupling pre- and post- compensators, and then having to deal only 
with separate SISO control loops (Dahlin et al, 1968 ; Owens, 1978 ; Rosenbrock, 1979; MacFarlane 1979). The development of self-tuning controllers based on state-space theory included SISO and MIMO systems under one general main framework. Adaptive schemes based on LQG control have been reported in the literature as schemes combining extended Kalman filtering (Jazwinski, 1970) and LQG (linear-quadratic-gaussian) control theory. (Athans, 1971). Peterka and Åström (1973) proposed a multivariable self-tuning regulator based on linear quadratic optimal control of processes with uncertain parameters. Ljung (1977) has developed a general procedure for analyzing recursive stochastic algorithms, which is useful in the study of the adaptive controllers. Borison (1979) extended the basic minimum variance self-tuning controller to the multivariable case. Koivo (1980) and Keviczky and Kumar (1981) extended Clarke and Gawthrop's method to a MIMO self-tuning controller. These first algorithms dealt only with square systems only where the number of inputs is the same as the number of outputs. Prager and Wellstead (1980) used a pole placement design procedure. Goodwin, Ramadge and Caines (1981) applied martingale theory to study an algorithm based on a modified stochastic approximation identification procedure and a minimum variance design. It was shown that, subject to a positive realness condition, the inputs and outputs are mean square bounded, and that the algorithm gives convergence to the optimal minimum variance controller. Some implicit and explicit LQG self-tuning schemes were studied by Aström (1980), and Grimble (1984). Some of them depend upon the solution of either a steady state Riccati equation or involved a minimum variance control law and a spectral factorization stage. Lam (1980) developed a statespace based self-tuner in which the numerical problems involved in iterating the Riccati equation were avoided. Problems arise when the number of inputs is not the same as the number of outputs and if different time delays exist between each input and output. Lam's results drew some attention in the recent research work on the adaptive control 
of MIMO adaptive stochastic systems, and algorithms of non-square systems have been derived (Goodwin et al, 1982 ; Grimble and Moir, 1983). Elliot and Wolovich (1982) put the above work into a more general framework by showing that the notion of the delay in the MIMO case was intimately related to the interactor matrix as introduced by Wolovich and Falb (1976). Goodwin and.Dugard (1983) finally treated a simple derivation of the minimum variance controller in the case of a general interactor matrix and a globally convergent algorithm was developed. The SISO extended-horizon predictive control scheme introduced by Ydstie (1982) was extended to MIMO systems by Dugard, Goodwin and Xianya (1984). Makila (1984) suggested an explicit adaptive method of solving an LQG control problem of stochastic systems with fixed structure regulators. The optimization of the regulator's parameters was achieved from the solution of a Ricatti-type equation (Anderson and Moore, 1971). Despite the number of proposed MIMO adaptive control schemes very few pilot-plant applications have been reported however (Toivonen, 1984; Martin-Sanchez and Shah, 1984).

In the past years, parameter identification of linear systems via orthogonal functions and polynomials has received some attention. Generally speaking, any signal can be considered as a signal vector in an infinite-dimensional signal space. The projection of the signal vector in a certain finite-dimensional subspace is then the least-square approximation of the signal by the basis functions spanning this subspace. And the error of this approximation is orthogonal to the subspace. One of the basic problems in signal representation is then to find a minimum-dimensional subspace in such a way that the information-bearing attributes of a specific class of signals are retained.

Historically, the use of orthogonal functions for obtaining approximations is well established. The approximating properties of Fourier series, the sine and cosine terms of which satisfy the orthonormality condition, are well known. The first application of other orthogonal sets to the transient problem was provided in 1932 by Y.W. Lee. For 
a causal system, such as the process loops under study, the impulse response may exist over the whole positive time axis. On this interval an appropriate, simple, convenient and well known orthogonal basis set is the Laguerre set (Lee, 1960). The Laguerre functions, a complete orthonormal set in $L_{2}[0, \infty)$, have been used often both for their convenient network realization and for their similarity to transient signals (Young and Huggins, 1961; King and Paraskevopoulos, 1977; Nurges and Jaaksoo, 1981 ; Dumont, Zervos and Bélanger, 1985). Wiener (1956) also proposed Laguerre functions for prediction and for non-linear systems. Other polynomial approximations of time functions have been used by many researchers to solve either continous or discrete control problems in recent years. In particular, the application of continuous Walsh polynomials to system identification was introduced by Corrington (1973) by constructing Walsh tables and by Chen and Hsiao (1975) who developed the Walsh integration operational matrix. The computational algorithm to calculate the expansion coefficients was simplified by using the derived integration operational matrix.

The Walsh operational method was applied to various problems such as the analysis, synthesis and optimization of time-invariant and time-varying systems, to bilinear systems, delay systems, distributed systems, multi- input-multi-output systems and also integral equations (for details see Horng and Ho, 1986). A parameter identification method of continuous-time systems, developed by expressing integral functions in terms of periodic input-output data suitable for implementation on a microprocessor by use of Walsh functions has been proposed by Bohn (1982). In order to facilitate digital image processing, the discrete Walsh series were developed by Kak (1974) to manipulate the integral transform characterization of patterns of a finite binary sequence. Other typical series applied to signal estimation, model-reduction problems, linear control problems and functional ordinary differential equations, are the Legendre (Chang and Wang, 1985), the Chebychev polynomials (Liu and Shih, 1984) and Jacobi 
polynomials (Lee, Tsay and Horng, 1986). Although effective recursive formulae were proposed, the computation was still tedious. The inversion of a high-dimensional matrix is often encountered in solving these problems. King and Paraskevopoulos (1979) applied the discrete Laguerre polynomials to solve the parametric identification problem. Hwang and Shih (1983) used the discrete Laguerre orthogonal polynomials and the discrete Chebyshev orthogonal polynomials, to solve the model reduction problem. Similarly, Horng and Ho (1985) applied the discrete Laguerre orthogonal polynomials and the discrete Chebyshev orthogonal polynomials to solve the discrete optimal control problem.

Most of the proposed methods in estimation and control that used orthogonal polynomials were essentially data compression works. The time domain data sequences (input and/or output) were transformed into discrete orthogonal polynomial spectra which are much shorter than the data sequences. Handling these shorter spectra, memory saving and computational advantage are obvious.

\subsection{Contribution of this thesis}

In this thesis the behaviour of several discrete self-tuning schemes based on the series representation of some orthonormal sets of functions is analyzed and implemented. The analysis, and the results obtained employing this new transfer function representation, are particularly favourable and they are considered to be well-suited to the adaptive control problems. It is our belief that this topic will inspire confidence in the adaptive control community in the potential use of orthonormal functions as a tool in estimation and control, and will also spur more research activity in the subject. Although the use of orthonormal functions is a very popular topic in estimation theory and signal processing, especially for the description of electrical signals, their use in adaptive control 
has not been much investigated. In this thesis we actually propose a method to develop a self-tuning adaptive control scheme based on orthonormal series representation. The use of three sets is investigated: (a) the set of Laguerre functions, (b) a modified set based on the Laguerres and (c) a set with complex poles. The results are encouraging and establish the succesful applicability of this new method. The reasons for using Laguerre functions are described later.

The contributions of this work to the theory of adaptive control are as follows:

- To introduce the orthonormal functions as a valuable tool in modelling plant dynamics and in the development of adaptive control algorithms. In particular the major features of the Laguerre set and two more others are investigated and their applicability is analysed.

- The demonstration of an analysis method for implementing adaptive control algorithms based on orthonormal sets of functions. The major advantages of the self-tuning schemes studied being: (a) the need of less a-priori knowledge about the order, the delay, the number of poles and zeroes and the phase characteristics of the plant, (b) the Finiteness property of the orthonormal sets as an advantageous way to increase on-line the number of filters for proper identification, (c) superior handling of time delays and non-minimum phase characteristics and (d) the flexibility and simple structure of the proposed algorithms that make them very attractive for industrial applications.

- The robustness properties of the new algorithms and their potential use in controlling plant dynamics efficiently are verified first by various computer simulations and second by an industrial application.

- To demonstrate the efficiency and applicability of the proposed schemes, the 
first industrial application of an adaptive self-tuning scheme using orthonormal functions is presented in detail.

Although Laguerre functions were chosen initially, because of their simplicity and their similarity to transient signals, other orthonormal functions such as Legendre functions may be used. The Laguerre functions exhibit strong advantages in identifying time-delays (a situation common in process control) because of their similarity to Padé approximants. As the model is expressed in a state-space form, state-space control design techniques may be used. A predictive control law is preferred because of its simplicity and ease of use. The common thread and novel aspect for all those schemes is the use of unstructured models based on orthonormal functions.

\subsection{Outline of the Thesis}

The idea of modelling and identifying linear systems using orthonormal functions, and especially using the Laguerre set is introduced in Chapter 2. Two deterministic selftuning control schemes, one explicit and one implicit, based on this approach and accompanied by some theoretical results, robustness issues, simulations results and a discussion on the practical aspects of the algorithm, are presented in Chapter 3 . The stochastic self-tuning control approach including some closed-loop stability and simulation results are considered in Chapter 4. A multivariable control scheme is developed in Chapter 5 and two simulation examples are given, including a papermachine headbox example. An industrial application based on the Laguerre-series self-tuning approach and performed on a $\mathrm{pH}$-control loop in a bleach plant at a pulp mill site is documented in Chapter 6. Chapter 7 deals with self-tuning control based on a modified Laguerre set of functions and also on a set of functions having complex exponential poles. 


\section{Chapter 2}

\section{Laguerre Functions in Modeling and Identification}

\subsection{Signal Description by Orthonormal Functions}

In the past years many different identification and parameter estimation methods for linear dynamic systems have been described in the literature (Ljung, 1987). In an effort to improve the operation and control of existing industrial processes and the design of new efficient control algorithms, an increasing interest was shown in the measurement of system dynamics. Any method which can give good measurement of the dynamics in the presence of plant disturbances within a reasonably short time with a small amount of data, and without significantly affecting the normal operation of the process, would be more attractive. The basis of estimation and identification consists of the mathematical description of the relation between the input and the output functions of the process under study. These functions are generally contaminated by noise, and the process dynamics may be subject to change from internal or external disturbances.

In dealing with the problem of identifying an unknown process in discrete time, two major decisions have to be taken in advance: (a) what mathematical model is to be used for the plant representation and (b) what estimation method is to be applied in order to find the unknown parameters. A common answer to (a) above is the well-known ARMAX mathematical model. Any of the proposed identification techniques in the literature deal with this mathematical model, and many of the self-tuning schemes are based on ARMAX series representation (Seborg et al, 1986). Other ways which have 
been proposed for modelling system dynamics include the impulse response description and the orthonormal functions representation. For the impulse response description a number of control schemes have been developed and some practical applications have been reported (Richalet et al, 1975 ; Rouhani and Mehra, 1982). Their philosophy can easily be extended to adaptive self-tuning schemes. As for the choice of estimation methods be applied there is a variety of choices. Correlation techniques, Least squares, Instrumental Variables, Maximum Likelihood, Stochastic Approximation, Gradient estimation techniques, Extended Least Squares, Weighted Least Squares, are some of the choices (Ljung , 1987). Each one has specific advantages; the simplest and most frequently used being the Least.Squares scheme.

Considered in this study are the use of orthonormal functions to represent system dynamics and the estimation method of Least Squares (Extended Least Squares) for parameter identification in the deterministic (stochastic) case. This combination provides strong features and, as it will be shown later, it resembles the Instrumental Variable identification method, well known for its robust properties (Trulsson, 1983).

This combination has also been used before with success in an off-line method for the optimal tuning of industrial PID controllers in process control, (Zervos, Bélanger and Dumont, 1985). Extended results from industrial trials have been reported in Dumont, Zervos and Bélanger (1984). The optimal PID tuning scheme also performed relatively well when it was compared to an adaptive predictive control scheme on the same $\mathrm{pH}$-loop of a bleach plant in a pulp and paper mill, (Dumont, Martin-Sanchez and Zervos, 1988).

If the approximation of the plant's impulse response $g(t)$ is carried out by developing the given function into an infinite expansion, (Kautz, 1954),

$$
g(t)=\sum_{i=1}^{\infty} r_{i} f_{i}(t)
$$


in terms of certain functions, $f_{i}(t)$, then by selecting as the approximation the first $N$ terms of this expansion;

$$
\hat{g}(t)=\sum_{i=1}^{N} r_{i} f_{i}(t)
$$

The constants $r_{i}$ are the coefficients of the expansion. The functions $f_{i}$ (which can be sums of damped exponentials and/or exponentially damped sinusoids, or polynomials, must be chosen so that,

- Convergence is rapid and uniform, thereby producing an accurate approximation for any fixed number of terms.

- The coefficients $r_{i}$ do not depend upon the number $N$.

- The calculation of the $N$ coefficients, $r_{i}$, is simple.

- The mean-squared error between $g(t)$ and $\hat{g}(t)$ is minimum for a given value of $N$.

Most of these properties are achieved by selecting the functions $f_{i}(t)$ to form an orthonormal set.

Some of the advantages of signal representation by orthonormal functions are,

- The coefficients of expansion can be determined by invoking orthonormality. A set of orthonormal functions can be generated from a set of linearly independent functions by using the Schmitt orthogonalization procedure (Stewart, 1973) and the normality condition.

- Good sensitivity behaviour in terms of changes of the function being approximated.

- Minimization of the mean squared error for a function to be approximated by a truncated linear combination of functions. 
- Wide application area in predicting or extrapolating signals.

A set of real and continuous functions $\left\{f_{1}(t), f_{2}(t), \ldots\right\}$ is said to be orthonormal in the range $[a, b]$ if,

$$
\int_{a}^{b} f_{i}(t) f_{j}(t) d t=\left\{\begin{aligned}
0 & \text { if } i \neq j \\
1 & \text { if } i=j
\end{aligned}\right.
$$

where, $i, j=1,2,3, \ldots$.

Completeness is another desirable fact apart from linear independence of the orthogonal functions. The orthonormal set $\left\{f_{i}(t)\right\}$ with,

$$
\int_{a}^{b} f_{i}^{2}(t) d t<\infty, \quad i=1,2, \ldots
$$

is called complete or closed in $\mathbf{L}_{2}[a, b]$ if for any continuous and real function $g(t)$ in $\mathbf{L}_{2}[a, b]$ and for any given real number $\epsilon>0$, there exists an integer $N$ such that,

$$
E=\int_{a}^{b}|g(t)-S(t)|^{2} d t<\epsilon
$$

where,

$$
S(t)=\sum_{i=1}^{N} \dot{r}_{i} f_{i}(t)=\underline{\mathbf{r}}^{T} \underline{\mathbf{f}}
$$

Where, $\underline{\mathbf{r}}^{T}=\left[\begin{array}{llll}r_{1} & r_{2} & \ldots & r_{N}\end{array}\right]$, and $\underline{\mathfrak{f}}^{T}=\left[\begin{array}{llll}f_{1} & f_{2} & \ldots & f_{N}\end{array}\right]$. The constants $r_{i}$ 's are called the spectrum gains and for deterministic signals can be computed from (Lee, 1960):

$$
r_{i}=\int_{a}^{b} g(t) f_{i}(t) d t
$$

For ergodic stochastic signals the integral in equation (2.6) is replaced by the expectation operator giving the cross-correlation at zero lag between the two signals, i.e. $r_{i}=E\left[g(t) f_{i}(t)\right]$.

An orthonormal set is said to be finite if when increasing the number of orthonormal filters in the signal representation of a specific square-integrable function from a low 
order to a high order; the low-order spectrum gains remain unchanged. The highorder approximation thus adds more terms for achieving a better approximation of the function and minimizing the error in the equation (2.4).

The approximation of a signal by a sum of a finite number of orthogonal functions in the minimum-integral-squared-error sense leads to the same coefficients as given above by equation (2.6); (Eykhoff, 1964). For an unknown function to be approximated, consider the Figure 2.1. The output $y(t)$ of a process is compared with a linear combination of $\left\{l_{i}(t)\right\}$, the outputs of the filters $F_{1}(s), F_{2}(s), \ldots, F_{N}(s) . w(t)$ represents any internal or external noise disturbances. If the input, $u(t)$, is a white stochastic stationary signal then the gains of the filters are given by the equation,

$$
\hat{r}_{i}=\frac{\mathbf{R}_{\mathbf{y}, \mathbf{l}_{\mathbf{i}}}(0)}{\mathbf{R}_{\mathbf{u}, \mathbf{u}}(0)}
$$

where the symbol $\mathbf{R}_{\mathbf{x}_{\mathbf{i}}, y_{\mathbf{i}}}(0)$ stands for the correlation function between the two signals $x_{i}(t)$ and $y_{i}(t)$ at zero time-lag. The numerator is the cross-correlation at zero lag between the output $y(t)$ and each filter's output and the denominator is the autocorrelation of the input (Zervos, Bélanger and Dumont, 1988). The orthogonality condition and the equation (2.7) are fullfilled only if the transfer functions of the filters are orthogonal to each other.

\subsection{The Laguerre functions}

\subsubsection{Introducing the Laguerre orthonormal set}

The Laguerre functions, a complete orthonormal set in $\mathrm{L}_{2}[0, \infty)$, have been used often because of their convenient network realization (Lee, 1960) and their similarity to transient signals. Their similarity to Padé approximants for time delay representations makes them particularly attractive for our purpose. All these remarkable features make 


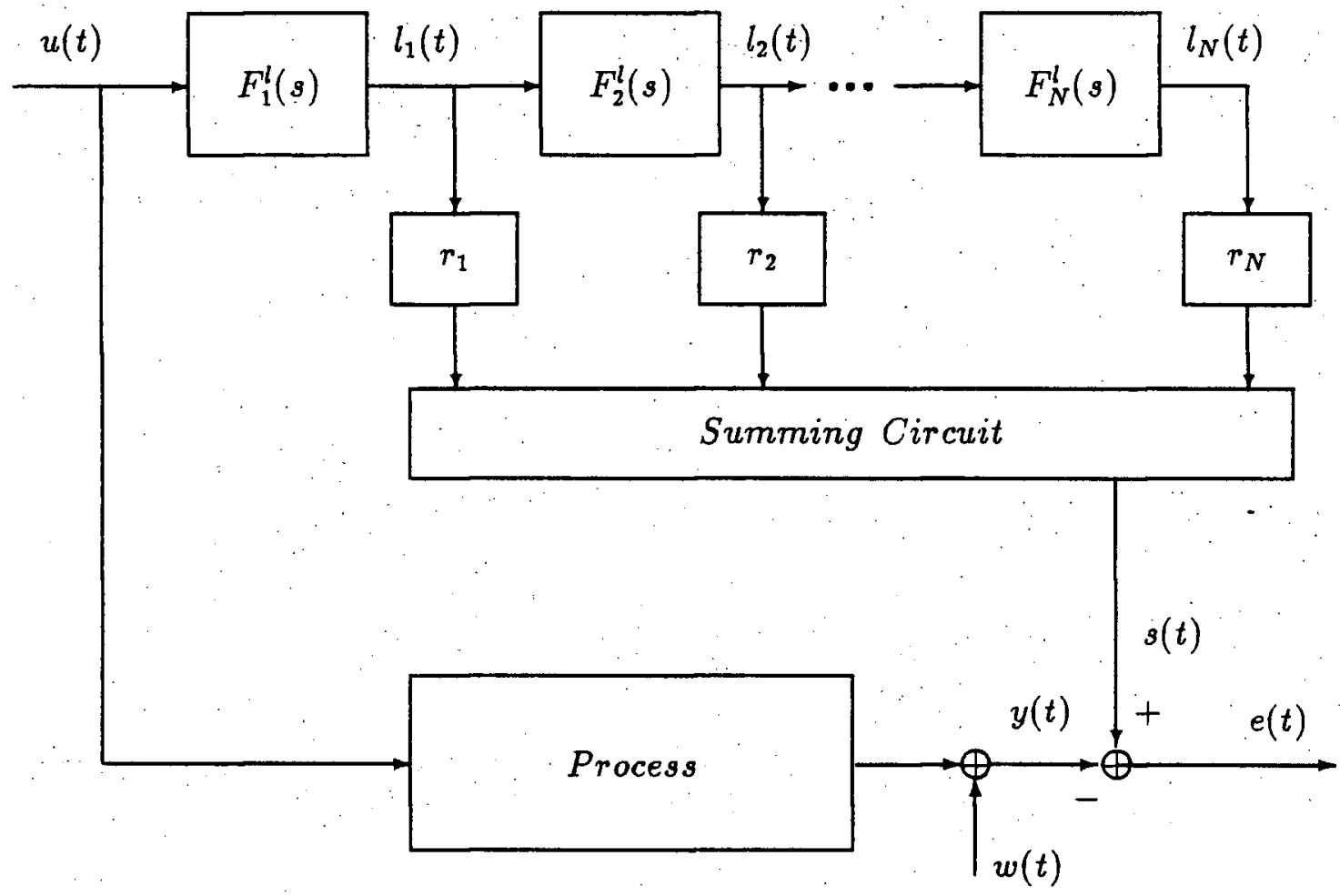

Figure 2.1: Approximation of a transfer function by a linear combination of orthonormal filters. 
the Laguerre functions a candidate of choice for use in identifying plant dynamics. Because of their good properties we plan also to incorporate them in the structure of an adaptive self-tuning control scheme.

In particular their advantages as a complete orthonormal set are summarized in short in the following :

- Advantageous and simple representation and flexible structure.

- Easy to model and construct.

- Similarity to transient signals that are common in process control.

- Similarity to Padé approximants providing superior representation and handling of time delays to the extent that it is possible to recover an estimate of the time delay during identification.

- They have been used before with success for transient signal representation (e.g. Lee, Young and Huggins, King and Paraskevopoulos, Nurges and Jaksoo, Dumont, Zervos and Bélanger).

- They have also been used for non-linear systems by Wiener who also recomended them for signal extrapolation and prediction.

In continuous time the Laguerre functions are described by (Lee,1960):

$$
\begin{aligned}
f_{i}(t) & =\sqrt{2 p} \frac{\exp (p t)}{(i-1) !} \frac{d^{i-1}}{d t^{i-1}}\left[t^{i-1} \exp (-2 p t)\right] \\
& =\sqrt{2 p}(-1)^{i-1} \exp (-p t) \mathcal{L}_{i-1}(2 p t)
\end{aligned}
$$

where $i$ is the order of the function $(i=1, . . N), p>0$ is the time-scale, and $\mathcal{L}_{i}(x)$ are the Laguerre polynomials, well-known in mathematical literature. These functions form 


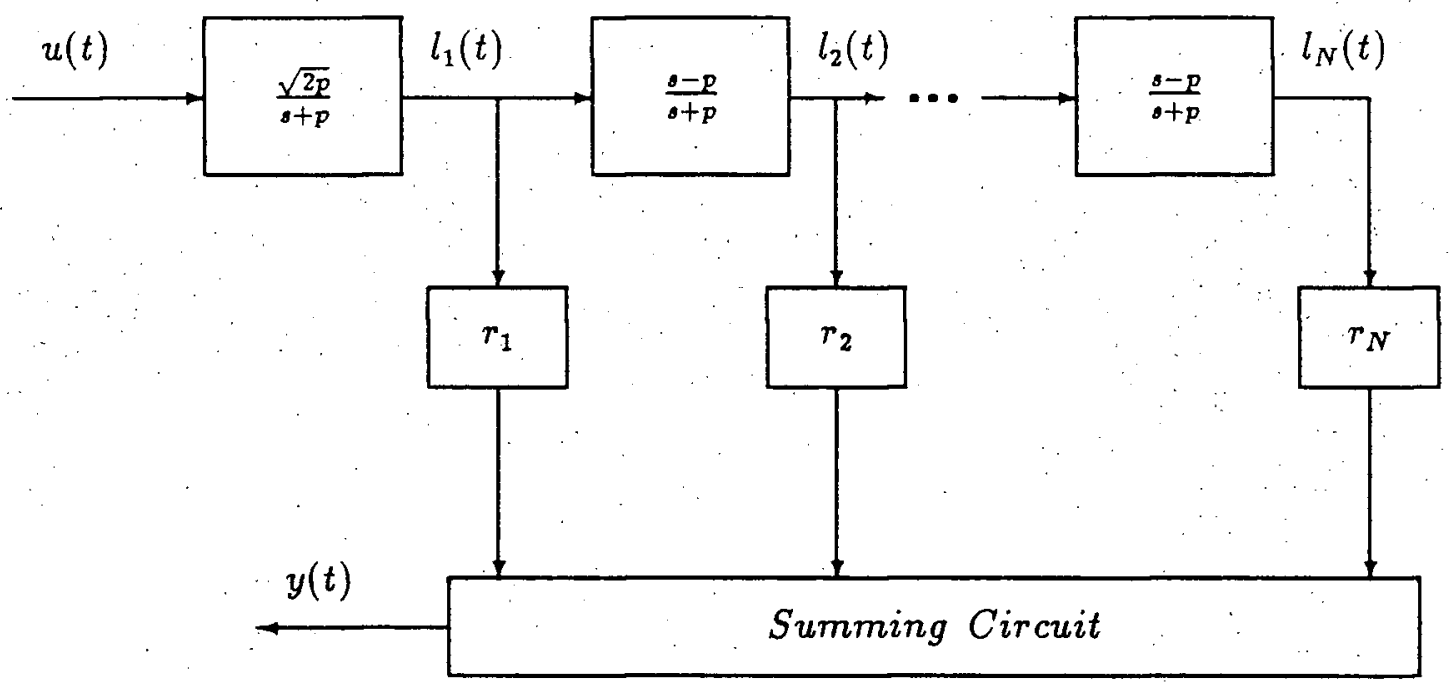

Figure 2.2: Laguerre Ladder Network.

an orthonormal set in the time-domain $[0, \infty]$ and the corresponding Laplace transform for this set is,

$$
F_{i}(s)=\sqrt{2 p} \frac{(s-p)^{i-1}}{(s+p)^{i}} \quad, \quad i=1, . ., N
$$

The orthonormality property from the time domain is preserved in the $s$-domain. This set is generated by the simple and convenient ladder network of Figure 2.2. The simplicity of the phase-shift chain in the Laguerre Network is a significant advantage for implementation.

Besides Laguerre, other useful real-pole sets may be derived from and related to 
other orthogonal polynomials, such as those of Tchebycheff, Legendre, and Jacobi. However, only the Laguerre set lends itself to a simplified determination of the coefficients $r_{i}$, (Kautz, 1954). Laplace-transforming equation (2.1) and substituting the expression for the Laguerre functions from equation (2.8) we get,

$$
G(s)=\sum_{i=1}^{N} r_{i} \sqrt{2 p} \frac{(s-p)^{i-1}}{(s+p)^{i}}
$$

Now; letting $(s-p) /(s+p)=w$, or $s=p(1+w) /(1-w)$ and rearranging the factors somewhat, gives:

$$
\sqrt{2 p} \frac{w}{(1-w)} G\left(p \frac{1+w}{1-w}\right)=\sum_{i=1}^{N} r_{i} w^{i}
$$

This expression may be regarded as the first $N$ terms of the power series expansion of the quantity on the left. That is, the first $N$ terms of the power series expansion,

$$
\left[\frac{(s-p)}{\sqrt{2 p}} G(s)\right]_{s=p(1+w) /(1-w)}=\sum_{i=1}^{N} r_{i} w^{i}
$$

may be computed numerically to determine the $r_{i}$ when the function $g(t)$ is analytically known.

\subsubsection{Least Squares Estimation of the Laguerre gains}

For identification purposes of a given $\mathbf{L}_{2}$ open-loop process it was shown in Section 2.1 that the Laguerre spectrum gains may be computed using simple correlation techniques. If the input is stationary white noise then the cross-correlations at zero lag between the individual filter outputs are all theoretically zero because of the orthogonality property in the orthonormal set. In that case the following proposition relates the spectrum gains of a transfer function with the spectrum gains of its output when the input is white noise.

Proposition 1 Consider the system in Figure2.1, where $\left\{f_{i}(t)\right\}$ is an orthonormal set of functions and $u(t)$ is a stochastic input signal. Assume the process's impulse response 
is $g(t)$ and its output is $y(t)$ and let their orthonormal expansions be,

$$
g(t)=\sum_{i=1}^{N} r_{i} f_{i}(t)
$$

with,

$$
r_{i}=\int_{0}^{\infty} g(t) f_{i}(t) d t
$$

and,

$$
y(t)=\sum_{i=1}^{N} c_{i} l_{i}(t)
$$

with,

$$
c_{i}=\mathbf{E}\left[y(t) l_{i}(t)\right]
$$

If $u(t)$ is stationary white noise with variance $\sigma^{2}$, then $c_{i}=\sigma^{2} r_{i}$.

Proof: Interchanging between the expectation and integral operators we have,

$$
\begin{aligned}
c_{i} & =\mathbf{E}\left[l_{i}(t) y(t)\right]=\mathbf{E}\left[\int_{0}^{\infty} f_{i}\left(t-\tau_{1}\right) u\left(\tau_{1}\right) d \tau_{1} \int_{0}^{\infty} g\left(t-\tau_{2}\right) u\left(\tau_{2}\right) d \tau_{2}\right] \\
& =\mathbf{E}\left[\int_{0}^{\infty} \int_{0}^{\infty} f_{i}\left(t-\tau_{1}\right) g\left(t-\tau_{2}\right) u\left(\tau_{1}\right) u\left(\tau_{2}\right) d \tau_{1} d \tau_{2}\right] \\
& =\int_{0}^{\infty} \int_{0}^{\infty} f_{i}\left(t-\tau_{1}\right) g\left(t-\tau_{2}\right) \mathbf{E}\left[u\left(\tau_{1}\right) u\left(\tau_{2}\right)\right] d \tau_{1} d \tau_{2} \\
& =\int_{0}^{\infty} \int_{0}^{\infty} f_{i}\left(t-\tau_{1}\right) g\left(t-\tau_{2}\right) \sigma^{2} \delta\left(\tau_{1}-\tau_{2}\right) d \tau_{1} d \tau_{2} \\
& =\sigma^{2} \int_{0}^{\infty} f_{i}\left(t-\tau_{1}\right) \int_{0}^{\infty} g\left(t-\tau_{2}\right) \delta\left(\tau_{1}-\tau_{2}\right) d \tau_{2} d \tau_{1} \\
& =\sigma^{2} \int_{0}^{\infty} f_{i}\left(t-\tau_{1}\right) g\left(t-\tau_{1}\right) d \tau_{1}=\sigma^{2} \int_{0}^{\infty} f_{i}(t) g(t) d t \\
& =\sigma^{2} r_{i} .
\end{aligned}
$$

where $\delta$ stands for the Kronecker delta.

The Laguerre filter gains may be computed using simple correlation functions as shown above but because in practice the computation of the correlation functions is 
done digitally and based on limited length of data, the use of least-squares parameter estimation is more efficient, (Zervos, 1984). Consider the Figure 2.1. At every time instant the output of the time-invariant plant $y(t)$ can be written as,

$$
y(t)=\sum_{i=1}^{N} r_{i} \int_{0}^{t} f_{i}(t-\tau) u(\tau) d \tau+w(t)
$$

letting,

$$
l_{i}(t)=\int_{0}^{t} f_{i}(t-\tau) u(\tau) d \tau
$$

then equation (2.16) becomes for the $k$ time-instant,

$$
y(k)=\sum_{i=1}^{N} c_{i} l_{i}(k)+w(k)=\underline{1}_{k}^{T} \underline{\mathrm{c}}+w(k)
$$

where $\underline{\underline{T}}_{k}^{T}=\left[\begin{array}{lll}l_{1}(k) & l_{2}(k) \ldots l_{N}(k)\end{array}\right]$, and $\underline{\mathbf{c}}^{T}=\left[\begin{array}{lll}c_{1} & c_{2} & \ldots \\ c_{N}\end{array}\right]$. Now, collecting data up to the $k$ sampling instant we get the vector-matrix form,

$$
\left[\begin{array}{c}
y(1) \\
y(2) \\
\vdots \\
y(k)
\end{array}\right]=\left[\begin{array}{cccc}
l_{1}(1) & l_{2}(1) & \cdots & l_{N}(1) \\
l_{1}(2) & l_{2}(2) & \cdots & l_{N}(2) \\
\vdots & \vdots & \vdots & \vdots \\
l_{1}(k) & l_{2}(k) & \cdots & l_{N}(k)
\end{array}\right]\left[\begin{array}{c}
c_{1} \\
c_{2} \\
\vdots \\
c_{N}
\end{array}\right]+\left[\begin{array}{c}
w(1) \\
w(2) \\
\vdots \\
w(k)
\end{array}\right]
$$

which can be put by inspection into the generalised linear form,

$$
\underline{\mathbf{y}}=M \underline{\mathbf{c}}+\underline{\mathbf{w}}
$$

For statistical and probabilistic considerations the number of observations $k$, needs to be much larger than the number $N$ of parameters to be estimated. Then the leastsquares estimate of the vector gain $\underline{\mathbf{c}}$ is given by the Normal equation (Eykhoff,1967; Strejc,1980),

$$
\hat{\mathbf{c}}_{L S}=\left(M^{T} M\right)^{-1} M^{T} \underline{\mathrm{y}}
$$

If we form explicitly the matrix $\left(M^{T} M\right)$ each entry will be of the form $:\left(M^{T} M\right)_{i j}=$ $\sum_{m=1}^{k} l_{i}(m) l_{j}(m)$, for $i, j=1, \ldots, N$. This matrix is diagonally dominant for large set 
of data because of the orthonormality of the Laguerre filters. As the number of data $k \rightarrow \infty$ the above matrix tends to be diagonal (a multiple of the unity matrix). If $u(t)$ is white noise with variance $\sigma^{2}$ and $\delta_{i j}$ is the Kronecker delta then we get for the elements of that matrix,

$$
\mathbf{E}\left[l_{i}(t) l_{j}(t)\right]=\sigma^{2} \delta_{i j} \simeq \lim _{k \rightarrow \infty} \frac{1}{k} \sum_{m=1}^{k} l_{i}(m) l_{j}(m)
$$

In the same way if we form explicitly the vector $\left(M^{T} \mathbf{y}\right)$ each entry will be of the form $\left(M^{T} \underline{\mathbf{y}}\right)_{i}=\sum_{m=1}^{k} l_{i}(m) y(m)$, for $i=1, \ldots, N$.

Computer simulations have indicated that when using least squares the general fit to the transfer function to be approximated is much better than use of the simple correlation approach described earlier and that fewer data points are required. Another interesting fact is the relation between the actual length of the parameter vector to be estimated and the number of available data points. The matrix $\left(M^{T} M\right)$ becomes nearly singular to working precision as the number of Laguerre filters increases and the number of data points decreases. An even more interesting fact is that the inverse of the above matrix can be pre-computed separately for a pre-determined input noise sequence and a fixed set of Laguerre filters, e.g. if the time-scale coefficient $p$ has always a fixed value and a time-scaling technique is used for the sampling time of an input of PRBS, (Zervos et al, 1985).

An obvious fact here is that by examining the matrix $\left(M^{T} M\right)$, the kind of leastsquares identification described above resembles very much the Instrumental Variable identification method which has proved to be quite robust (Ljung and Soderström, 1983). 


\subsubsection{Statistics of the estimates}

Any method for the identification of the parameters of an unknown plant requires experimental data. Due to observation noise or disturbances, the data may be provided with a specified tolerance. For the simple correlation method, in the estimation of the Laguerre gains, a crude check as to whether certain values of the cross-correlation functions could be effectively zero may be made by comparing the corresponding crosscorrelation estimates with their approximate standard errors obtained from a formula (Bartlett, 1955; Bendat and Piersol, 1966; Box and Jenkins, 1976).

For normal zero-mean signals, the "crude check" may be obtained (Zervos, 1984) using the formula;

$$
\operatorname{Var}\left[\boldsymbol{c}_{\mathbf{i}}\right]=1 / k^{2} \sum_{n=1}^{k} \sum_{m=1}^{k}\left[\mathbf{R}_{\mathbf{y}, \mathbf{l}_{\mathbf{i}}}(n-m) \mathbf{R}_{\mathbf{l}_{\mathbf{i}}, \mathbf{y}}(n-m)+\mathbf{R}_{\mathbf{y}, \mathbf{y}}(n-m) \mathbf{R}_{\mathbf{l}_{\mathbf{i}}, \mathbf{l}_{\mathbf{i}}}(n-m)\right]
$$

where the correlation functions estimates can be computed digitally using the usual approximative summation expression.

Since limited lengths of measurement data will be used over a finite time rather than over an infinite time, an expression for the standard error of the cross-correlation estimates would be useful in order to check how far from the true value the estimate may be. For normal stochastic input signals with zero mean value and variance of $\sigma^{2}$, the estimate of a Laguerre filter gain is given in discrete form by,

$$
\hat{r}_{i}=\frac{1}{\sigma^{2}} \hat{c}_{i}=\frac{1}{k \sigma^{2}} \sum_{n=1}^{k} y(n) l_{i}(n)
$$

where $k$ is the number of data that was used, and $i=1,2, \ldots, N$. In matrix-vector form for the whole parameter vector:

$$
\hat{\underline{\hat{\mathbf{f}}}}_{\mathrm{cor}}=\frac{1}{k \sigma^{2}} M^{T} \underline{y}
$$

where $M$ was defined from equations (2.19),(2.20). Now using equation (2.24) and the assumption that the plant noise is uncorrelated with the Laguerre filter outputs 
(Figure 2.1), the expected value of the parameter vector given the input gives,

$$
\begin{aligned}
\mathbf{E}\left[\underline{\hat{\mathbf{r}}}_{\mathrm{cor}} / M\right] & =\frac{1}{k \sigma^{2}}\left\{\mathbf{E}\left[\left(M^{T} M\right) \underline{\mathbf{r}}\right]+\mathbf{E}\left[M^{T} \underline{\mathbf{w}}\right]\right\} \\
& =\frac{1}{k \sigma^{2}} \mathbf{E}\left[\left(M^{T} M\right) \underline{\mathbf{r}}\right] \\
& \neq \underline{\mathbf{r}}
\end{aligned}
$$

This means that the simple correlation estimates of the Laguerre gains, given the input, are biased. Clearly for large number of data $(k \rightarrow \infty)$ the matrix $\left(M^{T} M\right) \rightarrow\left(\sigma^{2} I\right)$, where $I$ is the identity matrix. However over all possible inputs the expected value of the simple correlation estimate of a Laguerre gain is unbiased as shown below;

$$
\begin{aligned}
\mathbf{E}\left[\hat{r}_{i}\right] & =\frac{1}{k \sigma^{2}} \sum_{n=1}^{k} \mathbf{E}\left[y(n) l_{i}(n)\right] \\
& =\frac{1}{k \sigma^{2}} \sum_{n=1}^{k} \mathbf{R}_{\mathbf{y}, \mathbf{l}_{\mathbf{i}}}(0)=\frac{1}{k \sigma^{2}} \mathbf{R}_{\mathbf{y}, \mathbf{l}_{\mathbf{i}}}(0)=r_{i}
\end{aligned}
$$

Rather than calculating the variance of every single estimate of the Laguerre gains; may as well just show what the typical standard error (e) of the gain vector looks like for various $k$ and possibly $N$. Better still,

$$
\underline{\mathbf{e}}=\mathbf{E}\left[\hat{\underline{\mathbf{r}}}_{\text {cor }} / M\right]-\underline{\mathbf{r}}=\left\{\frac{1}{k \sigma^{2}} M^{T} M-I\right\} \underline{\mathbf{r}}
$$

In general; given,

$$
\underline{\mathbf{e}}=M^{\prime} \underline{\mathbf{r}}
$$

where $M^{\prime}$ is the matrix inside the curly brackets in equation (2.25), we have,

$$
\lambda_{\min }\left(M^{\prime T} M^{\prime}\right)|\underline{\mathbf{r}}|^{2} \leq|\underline{\mathbf{r}}|^{2} \leq \lambda_{\max }\left(M^{\prime T} M^{\prime}\right)|\underline{\mathbf{r}}|^{2}
$$

where $\lambda$ stands for the eigenvalue of a matrix. If the matrix is symmetric then, $\left(M^{\prime T} M^{\prime}\right)=M^{\prime 2}$ and $\lambda\left(M^{\prime T} M^{\prime}\right)=\lambda^{2}\left(M^{\prime}\right)$, and we finally get,

$$
\left|\lambda_{\min }\left(M^{\prime}\right)\right| \leq \frac{|\underline{\mathbf{e}}|}{|\underline{\mathbf{r}}|} \leq\left|\lambda_{\max }\left(M^{\prime}\right)\right|
$$


This result shows that the error in the estimates is always bounded depending on the number of data $(k)$ and the number of filters $(N)$. To apply this, one has to calculate the minimum and the maximum eigenvalue for the matrix $M^{\prime}$ and use equation (2.28). Both should of course go to zero for large $k$. For finite $k$, they give a good indication of the error in the expected value.

For the Least Squares parameter estimation case, the estimates of the Laguerre gains appear actually to be unbiased. Using equations (2.20) and (2.21) one can easily get the following result for the expected value of the parameter vector given the input,

$$
\begin{aligned}
\mathbf{E}\left[\hat{\mathbf{c}}_{L S} / M\right] & =\mathbf{E}\left[\left(M^{T} M\right)^{-1} M^{T}(M \underline{\mathbf{c}}+\underline{\mathbf{w}})\right] \\
& =\underline{\mathbf{c}}+\mathbf{E}\left[\left(M^{T} M\right)^{-1} M^{T} \underline{\mathbf{w}}\right] \\
& =\underline{\mathbf{c}}
\end{aligned}
$$

It is worthwhile to mention here that the above is true even if the noise $w(t)$ corrupting the output of the plant is coloured, as long as it is uncorrelated with the filter outputs. Suppose that a system at the $k^{\text {th }}$ sampling instant is described by,

$$
y(k)=\sum_{i=1}^{N} c_{i} l_{i}(k)+w(k)=\underline{\mathbf{T}}_{k}^{T} \underline{\mathbf{c}}_{0}+w(k)
$$

where $\underline{1}_{k}^{T}=\left[\begin{array}{lll}l_{1}(k) & l_{2}(k) \ldots l_{N}(k)\end{array}\right]$, and $\underline{\mathbf{c}}_{0}^{T}=\left[\begin{array}{lll}c_{1} & c_{2} \ldots c_{N}\end{array}\right]$. Collecting data up to the $k^{\text {th }}$ sampling instant we get the vector-matrix equation (2.20), i.e.,

$$
\underline{\mathrm{y}}=M \underline{\mathrm{c}}_{0}+\underline{\mathrm{w}}
$$

Now multiplying both sides of the above equation by $\left(\frac{1}{k} M^{T}\right)$ we get,

$$
\left(\frac{1}{k} M^{T}\right) \underline{\mathbf{y}}=\left(\frac{1}{k} M^{T} M\right) \underline{c}_{0}+\left(\frac{1}{k} M^{T}\right) \underline{\mathbf{w}}
$$

And finally for the parameter vector we get,

$$
\underline{\mathfrak{c}}_{0}=\left(\frac{1}{k} M^{T} M\right)^{-1}\left(\frac{1}{k} M^{T}\right) \underline{\mathbf{y}}+\left(\frac{1}{k} M^{T} M\right)^{-1}\left(\frac{1}{k} M^{T}\right) \underline{\mathbf{w}}
$$


If we define the following,

$$
R(k)=\frac{1}{k}\left(M^{T} M\right), \quad \underline{h}(\dot{k})=\frac{1}{k}\left(M^{T} \underline{\mathbf{y}}\right)
$$

then equation (2.31) can also be written as,

$$
\underline{h}(k)=R(k) \underline{\mathbf{c}}_{0}+\underline{\epsilon}(k)
$$

Now the following proposition as in Trulsson (1983) on the open-loop identification of a system is presented using the least-squares technique.

Proposition 2 Suppose that $\underline{z}(t)$ is an instrumental variable vector of the form

$$
\underline{\underline{z}}^{T}(t)=\left[\begin{array}{llll}
l_{1}(t) & l_{2}(t) & \ldots & l_{N}(t)
\end{array}\right]
$$

and is such that,

(a)

$$
\underline{\epsilon}(k)=\frac{1}{k} \sum_{m=1}^{k} \underline{z}(m) w(m) \rightarrow \underline{0}, \quad \text { as } \quad k \rightarrow \infty \quad \text { w.p.1 }
$$

(b) With probability 1 the smallest singular value of $R(k)$ does not tend to zero,

$$
\sigma_{\min }[R(k)] \nrightarrow 0 .
$$

Suppose also that either of the following exists and is known

(a) A lower bound $\delta_{0}$ of

$$
\underset{k \rightarrow \infty}{\limsup }\left[\sigma_{\min }[R(k)]\right]
$$

or

(b) A function $\gamma(k)$ such that

$$
\underline{\epsilon}(k) / \gamma(k) \rightarrow 0 \quad \text { as } \quad k \rightarrow \infty \quad \text { w.p.1, and } \quad \gamma(k) \rightarrow 0 \quad \text { as } \quad k \rightarrow \infty .
$$


Then it is possible to construct a sequence $\{\underline{\hat{\hat{c}}}(k)\}$ with $\operatorname{dim} \underline{\hat{\mathbf{c}}}=\operatorname{dim} \underline{\mathbf{c}}_{0}$, such that,

$$
\left|\hat{\mathbf{c}}(k)-\underline{\mathbf{c}}_{0}\right| \rightarrow 0 \quad \text { as } \quad k \rightarrow \infty \quad \text { w.p.1. }
$$

Proof: Define $\hat{\mathbf{c}}(k)$ as,

$$
\hat{\hat{\mathbf{c}}}(k)=R^{-1}(t(k)) \quad h(t(k))
$$

where $t(k)$ is the largest integer $t \leq k$ such that,

$$
\begin{aligned}
& \sigma_{\min }(R(t))>\delta_{0} / 2 \quad \text { in case }(2.38) \\
& \sigma_{\min }(R(t))>\gamma(t) \quad \text { in case }(2.39)
\end{aligned}
$$

From equation (2.34) and equations $(2.42),(2.43)$, we can write,

$$
\begin{aligned}
|\hat{\mathbf{c}}(k)| & \equiv\left|\underline{\hat{\mathbf{c}}}(k)-\underline{\mathbf{c}}_{0}\right|=\left|R^{-1}(t(k)) \underline{\epsilon}(t(k))\right| \\
& \leq\left[\sigma_{\min }(R(t(k)))\right]^{-1}|\underline{\epsilon}(t(k))| \\
& \leq \begin{cases}\frac{2}{\delta_{0}}|\underline{\epsilon}(t(k))| & \text { in case (2.38) } \\
(\gamma(t(k)))^{-1}|\underline{\epsilon}(t(k))| & \text { in case (2.39) }\end{cases}
\end{aligned}
$$

From equations (2.36), (2.37) and equation (2.44) it can be seen that equation (2.40) is true if $t(k) \rightarrow \infty$ as $k \rightarrow \infty$. In case of equation (2.38) this follows from equation (2.42), and in case of equation (2.39) this follows from equation (2.43).

The conditions described by equations (2.36) and (2.37) define the desired properties of the instrument vector $\{\underline{z}(t)\}$. The instruments satisfy these properties because they are uncorrelated with the noise $\{w(t)\}$, by the way they were defined in equation (2.35), and because $\underline{z}(t)=\underline{l}(t)$, they guarantee the validity of the condition in equation (2.37). The analysis so far was made under the assumption that no feedback input is used. If 
however a feedback input is used then an external white noise process $v(t)$ independent of $w(t)$ must be injected also in the system as it was suggested in Trulsson (1983). A price is paid here for the additional noise disturbance, input but this can be made so small that it has only a small influence on the closed-loop behaviour.

\subsubsection{The Discrete-time Laguerre set}

So far the continous-time Orthonormal Laguerre set has been discussed. The discrete form of the orthonormal Laguerre set is particularly important for use with digital computers when it comes to implementation. This requires the transformation of the orthonormal functions from the continuous form into the sampled-data form. One apparent solution would be to apply direct Z-transform, however the Z-transform of continuous orthonormal exponentials are not themselves orthogonal in Z-domain (Young and Huggins, 1962). This is due to the fundamental property of sampling that a signal with a frequency spectrum higher than the sampling frequency is not exactly reproducible from its sampled data. The Z-transforms of a signal involves the values of the signal at equal-spaced instants separated by the sampling interval, $T=2 \pi / \omega$, where $\omega$ is the sampling angular frequency. According to the sampling theorem only a signal having a frequency spectrum lower than $\omega$ is exactly reproducible from the sampled data. However the exponential functions, which vanish identically over half of the time-axis, have a non-zero spectrum extending over almost all frequencies. As a result orthogonality in the s-domain does not yield orthogonality in the z-domain representatives of these functions. Also the iterative procedure used in continuous time to define the $N^{\text {th }}$ Laguerre filter is not guaranteed to hold in discrete time after the Z-transformation.

In the present study the discretization of the Laguerre Ladder Network is accomplished by the continuous network compensation method (Jury,1958) for each of the 
blocks in Figure 2.2. In this way the properties of the continuous-time orthonormal set are preserved while expressing them efficiently in discrete time. The simple, flexible and convenient forms the functions have in continuous time while working in discrete time are also kept. This method involves the use of linear networks in either the forward or the feedback path of a sampled-data system. Consider for example Figure 2.3. The input to the block $G_{h}(s)$ consists of a set of impulse samples of $u(t)$ and its output is continuous and fed continuously to the plant $G(s)$. The network compensator can be determined from the desired time-response qualities, for instance to describe a continuous output whose sampled values coincide with the sampled response of the sampled-data control system (Nease, 1957). The fictitious interpolator $G_{h}(s)$ in this case is employed to produce a piece-wise straight-line approximation of the continuous signal between successive sampling instants. This method is based on the fact that $G(g)$ has an impulse response which is. well represented by straight lines between sampling instants, a fact which is usually true for most of the process control loops encountered in industry. Thus the transformed sampled-data control system output coincides with the sampled values of the continuous system output at the sampling instants. Another possibility for improving the approximation is to use second or higher-degree intrerpolators which give better smoothing properties. The goal is to obtain a highly accurate representation without increasing mathematical complexity. By using the above proposed discretization method the signal representations are kept accurate while having simple mathematical expressions.

The function of the fictitious hold $G_{h}(s)$ is to reconstruct as much as possible the continuous function being sampled. If $G_{h}(s)$ is chosen so that its output is a straightline approximation between successive sampling points then the transfer function of 


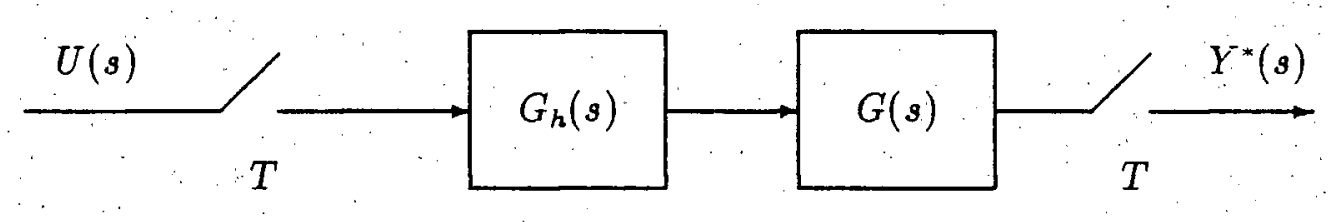

$G^{\star}(s)$

Figure 2.3: The Continuous Network Compensation of $G^{*}(s)$

such a hold is,

$$
G_{h}(s)=\frac{e^{T s}-2+e^{-T s}}{T s^{2}}
$$

The impulse response of such a filter which has a triangular shape is shown in Figure 2.4.

By inspection, this kind of linear triangle-hold interpolator provides a polygonal approximation of the output function. The function in equation (2.45) is not physically realizable since the shape of its impulse response from Figure 2.4 reveals a non-causal function. However, when it is cascaded with a continuous-time plant $G(s)$ then it is possible to evaluate the Z-transform of the overall system, which is physically realizable. Looking at the Laguerre ladder network in Figure 2.2 for process signal synthesis the discretization is done in the following way. If $T$ is the sampling period then the first input box,

$$
F_{1}^{l}(s)=\frac{\sqrt{2 p}}{(s+p)}
$$

is preceded by a zero-order hold and then it is Z-transformed to give the discrete-time 


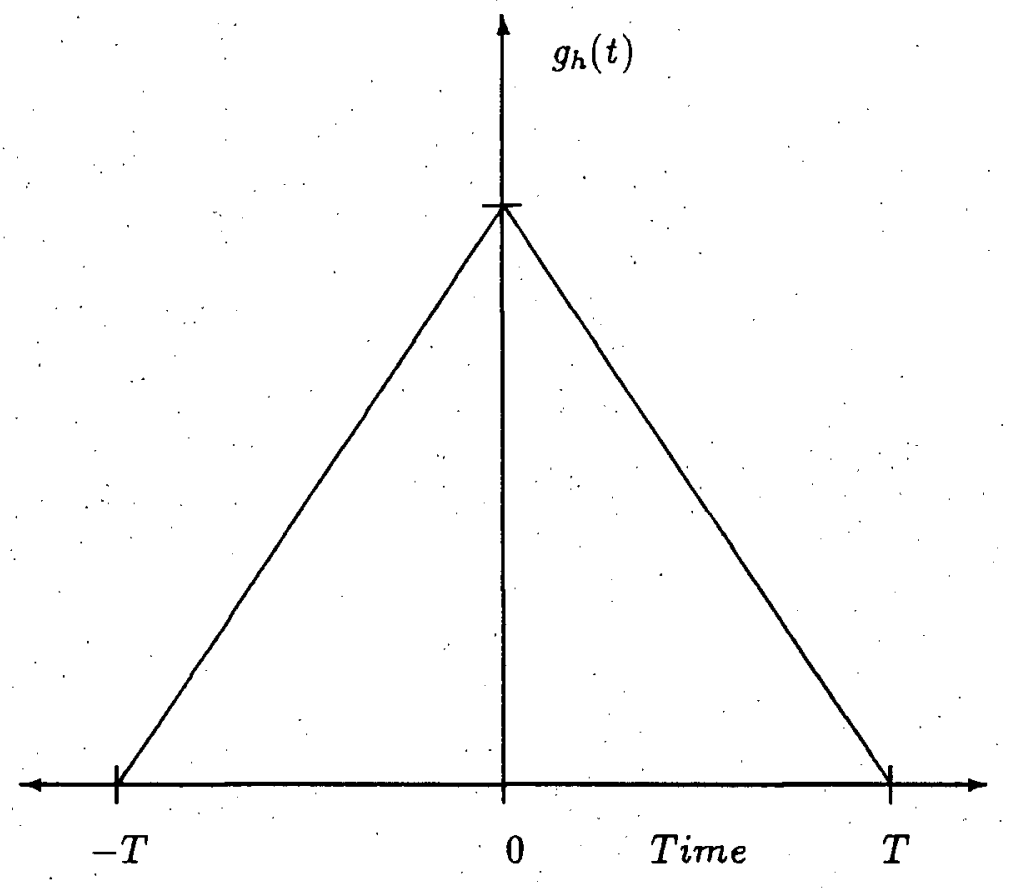

Figure 2.4: Impulse response of a triangle-hold $G_{h}(s)$

transfer function,

$$
F_{1}^{l}(z)=\frac{\sqrt{2 p}}{p} \frac{\left(1-e^{-p T}\right)}{\left(z-e^{-p T}\right)}
$$

Each of the remaining similar section boxes in Figure 2.2 is a phase-shift all-pass $1^{\text {st }}$ order filter which is discretized with the use of the triangle-hold linear interpolator filter given by equation (2.45), giving the discrete-time transfer function,

$$
F_{i}^{l}(z)=-\frac{z\left(T+2\left(e^{-p T}-1\right) / p\right)-T e^{-p T}-2\left(e^{-p T}-1\right) / p}{T\left(z-e^{-p T}\right)} \quad \text { for } i>1
$$

If we define,

$$
\begin{gathered}
\tau_{1}=e^{-p T} \\
\tau_{2}=T+\frac{2}{p}\left(e^{-p T}-1\right) \\
\tau_{3}=-T e^{-p T}-\frac{2}{p}\left(e^{-p T}-1\right)
\end{gathered}
$$




$$
\tau_{4}=\sqrt{2 p} \frac{\left(1-\tau_{1}\right)}{p}
$$

then the difference input-output equation for each of the outputs $l_{i}(t)$ of the Laguerre ladder network is given by,

$$
\begin{aligned}
l_{i}(t) & =(-1)^{i-1} \tau_{2}^{i-2}\left(\tau_{1} \tau_{2}+\tau_{3}\right) / T^{i-1} l_{1}(t-1) \\
& +(-1)^{i-2} \tau_{2}^{i-3}\left(\tau_{1} \tau_{2}+\tau_{3}\right) / T^{i-2} \quad l_{2}(t-1) \\
& +\cdots+(-1)\left(\tau_{1} \tau_{2}+\tau_{3}\right) / T l_{i-1}(t-1) \\
& +\tau_{1} l_{i}(t-1) \\
& +(-1)^{i-1} \tau_{2}^{i-1} \tau_{4} / T^{i-1} u(t-1)
\end{aligned}
$$

\subsection{Modelling of dynamic systems}

Described so far has been the Laguerre orthonormal set of functions and their important role in systems identification has been stated. The convenient representation of the Laguerre ladder network as shown in Figure 2.2 can be expressed in several mathematical ways. For our purpose it is convenient to represent it in a state-space form. This will enable us to derive predictive expressions of plant outputs in a straightforward manner. The outputs $l_{i}(t),(i=1, \cdots, N)$ from each block in Figure 2.2 are taken to be the states of the Laguerre ladder network. Defining the state vector as

$$
\underline{l}^{T}(t)=\left[\begin{array}{llll}
l_{1}(t) & l_{2}(t) & \ldots & l_{N}(t)
\end{array}\right]
$$

then by discretizing each block as it was described by equations $(2.47),(2.48)$ and using equation ((2.49) a discrete-time state space representation of the Laguerre network can be readily available in the form,

$$
\underline{l}(t+1)=A \underline{l}(t)+\underline{b} u(t)
$$


where $\underline{l}(t)$ is the $N$-dimensional state vector, and $u(t)$ is the system input. $A$ is a lower triangular $N \times N$ matrix where the same elements are found respectively across the diagonal or every subdiagonal. If $T$ is the sampling period then,

$$
A=\left[\begin{array}{cccc}
\tau_{1} & 0 & \ldots & 0 \\
\frac{-\tau_{1} \tau_{2}-\tau_{3}}{T} & \tau_{1} & \cdots & 0 \\
\vdots & \vdots & \vdots & \vdots \\
\frac{(-1)^{N-1} \tau_{2}^{N-2}\left(\tau_{1} \tau_{2}+\tau_{3}\right)}{T^{N-1}} & \cdots & \frac{-\tau_{1} \tau_{2}-\tau_{3}}{T} & \tau_{1}
\end{array}\right]
$$

and

$$
\underline{b}^{T}=\left[\begin{array}{llll}
\tau_{4},\left(-\tau_{2} / T\right) \tau_{4} & \cdots & \left(-\tau_{2} / T\right)^{N-1} \tau_{4}
\end{array}\right]
$$

The above state-space system is stable $(p>0)$, observable, and controllable. The output of the process to be modelled is then approximated by the weighted sum of the outputs of the Laguerre filters having the form,

$$
y(t)=\underline{c}^{T} \underline{l}(t)
$$

The above weighted sum in equation (2.54) can be taken as the projection of the plant output onto the linear space whose basis is the orthonormal set of Laguerre functions.

The standard calculation of the Laguerre spectrum gains employs equation (2.6) using well-known correlation techniques. A more efficient way is to use the least-squares parameter estimation method described in Section 2.2.2. In the open-loop case, the identification can be performed by exciting the system with a white noise sequence, i.e. both the system shown in Figure 2.2 and the system whose impulse response is $g(t)$ are excited by a white noise sequence (or PRBS), and $k$ output sample-points are collected. Then in the least-squares sense the normal equations can be used in vectormatrix form as given by equation (2.21). The parameters obtained in that way were proved in Section 2.2.3 to be unbiased even if the output of the plant was corrupted 
by coloured noise. If the identification is performed under closed-loop operation, as it is the case when a self-tuning scheme is employed, then the output of the controller is not a white noise sequence. However input-output data can still be collected for online plant identification. Simulations have shown that this least-squares identification technique produces very good results.

An advantage using an orthonormal series representation to identify a plant is that when the identification order is increased then the low-order coefficients stay practically constant. Thus, the model order can be changed on-line with minimal transient. On the other hand, for an ARMAX model, increasing the model order means change in all parameters and thus the identification procedure goes through a significant transient period. Another advantage is that time-delays can be easily modeled since this approach does not require distinction from the actual plant dynamics. As a consequence an estimate of the delay can also be derived. Laguerre functions show very strong features in this situation because of their similarity to Padé approximants. Results from the following simulation example outline both the above advantages.

Example 2.1 : Consider the following underdamped $(\zeta=0.45)$ second-order continuous-time plant that contains also a significant time-delay $\left(k_{d}=10\right)$ within its dynamics,

$$
G(s)=\frac{Y(s)}{U(s)}=\frac{0.25 e^{-10 s}}{\left(s^{2}+0.45 s+0.25\right)}
$$

Assume that all time units are in seconds. The natural angular frequency of the secondorder plant is $\omega_{0}=0.5 \mathrm{rad} / \mathrm{s}$ and its settling time is $\sim 25$ secs.

The above plant, including its long time-delay, was simulated in continuous time, using ACSL, and its input was excited with 5 identical periods of DIBN (Discrete Interval Binary Noise; Chow and Davies, 1964) sequences, each one of 64 samples periods, for a total of 320 points. The sampling time of the DIBN sequence was 
$2 \mathrm{sec}$, its absolute amplitude was 1 (i.e. of standard error $\sigma=1.0$ ) and the data points from the plant's output were collected every $2 \mathrm{sec}$, for a total of 320 points. No noise disturbance was added to the output of the plant. Upon collection of the data an identification experiment was performed using the already described Least-squares Laguerre functions method. The number of Laguerre functions used was $N=12$ and their time-scale was $p=0.5$. The 12 identified Laguerre spectrum gains are given in the first column of Table 2.1. The step responses of the true plant and the identified one are given in Figure 2.5. The two responses are almost identical. The time delay of $k_{d}=10$ and the underdamping second-order dynamics have been very well represented with the use of just the 12 weighted Laguerre functions. If one used the correlation method to find the Laguerre gains (which nevertheless would give biased estimates) then 3000 points would not have been enough even to get a proper identification (see Zervos, 1984, Experiment 1).

As a second experiment the output of the plant was corrupted with correlated noise before performing the identification. White noise was passed through a first-order linear transfer function of the form $G_{n}(s)=(0.2 s+1) /(s+1)$, and then was added to the plant's output. Everything else remained the same as in the first experiment above. Two identification experiments were performed: (a) One with Standard Error of 0.5 , and (b) one with Standard Error of 0.8. The 12 identified Laguerre spectrum gains for each case are given respectively in Table 2.1. The second and third columns contain the gains for the case with output noise, $\sigma=0.5$ and $\sigma=0.8$, respectively. The step responses of the true plant and the identified ones are shown in Figure 2.6. The responses of the identified plants match the response of the true plant very well despite the corruption of the plant's output by correlated noise. 


\begin{tabular}{|c||c|c|c|}
\hline \multicolumn{4}{|c|}{ Identified Laguerre Gains } \\
\hline \hline & $\begin{array}{c}\text { No output } \\
\text { noise }\end{array}$ & $\begin{array}{c}\text { Noise } \\
\sigma=0.5\end{array}$ & $\begin{array}{c}\text { Noise } \\
\sigma=0.8\end{array}$ \\
\hline$c_{1}$ & 0.000795 & 0.003251 & 0.004725 \\
\hline$c_{2}$ & -0.014922 & -0.015017 & -0.015074 \\
\hline$c_{3}$ & 0.101046 & 0.088314 & 0.080674 \\
\hline$c_{4}$ & -0.317984 & -0.323456 & -0.326739 \\
\hline$c_{5}$ & 0.362282 & 0.376566 & 0.385135 \\
\hline$c_{6}$ & 0.197190 & 0.202946 & 0.206398 \\
\hline$c_{7}$ & -0.388695 & -0.386242 & -0.384770 \\
\hline$c_{8}$ & -0.381893 & -0.385412 & -0.387523 \\
\hline$c_{9}$ & -0.011724 & -0.018451 & -0.0224867 \\
\hline$c_{10}$ & 0.156156 & 0.151452 & 0.148630 \\
\hline$c_{11}$ & 0.093519 & 0.088498 & 0.085485 \\
\hline$c_{12}$ & 0.019635 & 0.019029 & 0.018666 \\
\hline
\end{tabular}

Table 2.1: The values of the 12 identified Laguerre gains for each case.

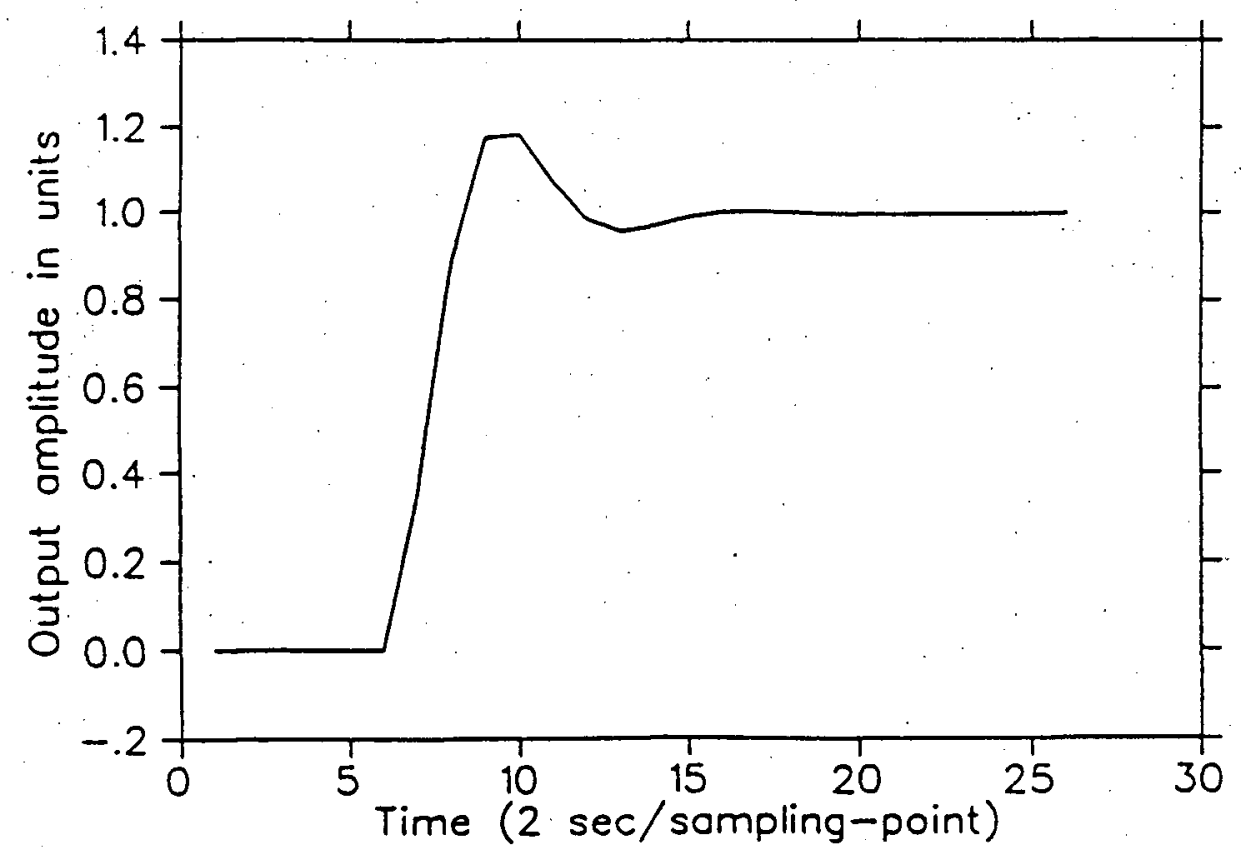

Figure 2.5: Example 2.1 : Unit step responses of the true plant and the identified one for the case without output noise (The 2 curves are identical). 


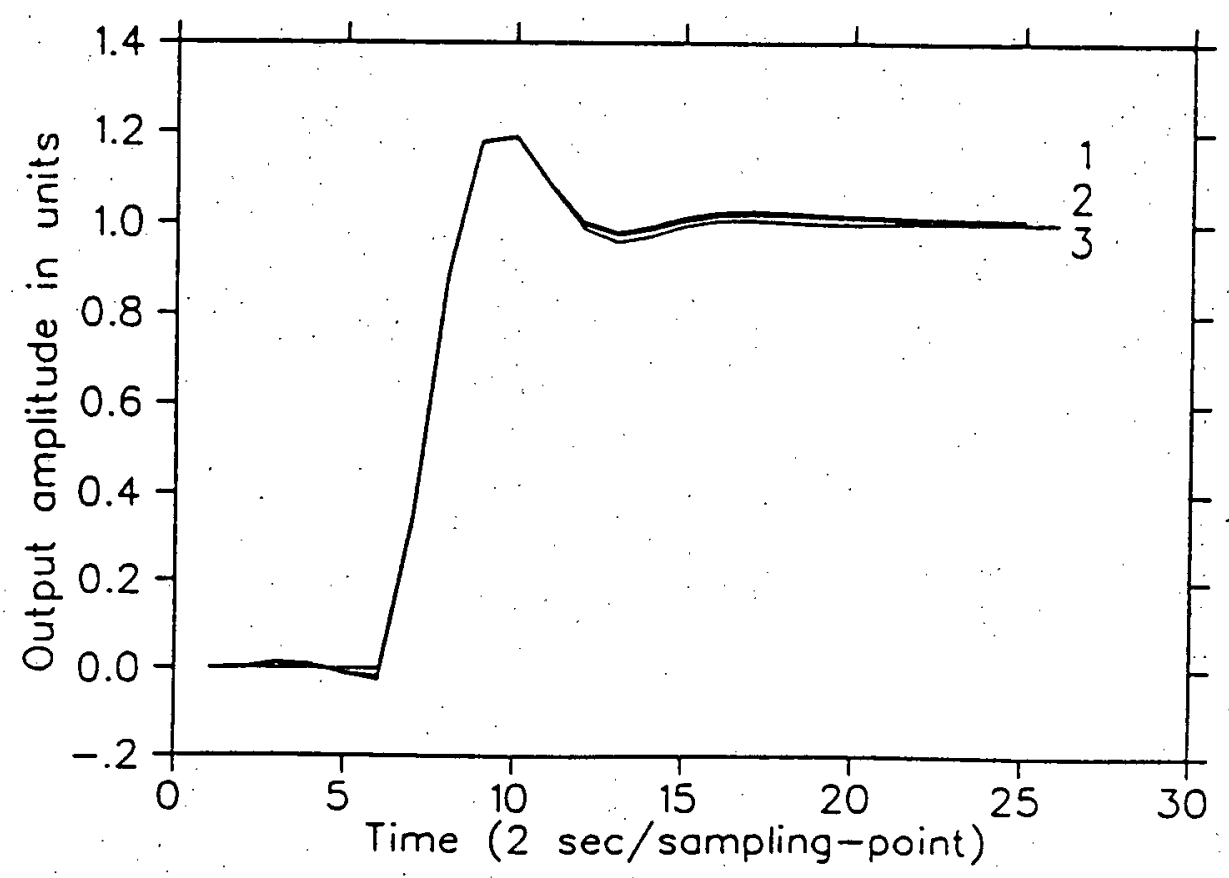

Figure 2.6: Example 2.1 : Unit step responses of the true plant (3), the identified one when $\sigma=0.5(2)$, and when $\sigma=0.8(1)$. 


\section{Chapter 3}

\section{Deterministic Adaptive Control}

\subsection{The Predictive Control Law}

As was first suggested by Dumont and Zervos (1986) the derived state-space representation of the orthonormal set of Laguerre functions presented in Section 2.3 can be used as the basis to formulate a predictive expression for the plant output. Simple Predictive Control concepts can then be employed to construct an adaptive self-tuning control scheme. Recalling from before, the state-space representation of the Laguerre Ladder Network is of the form,

$$
\begin{gathered}
\underline{l}(t+1)=A \underline{l}(t)+\underline{b} u(t) \\
y(t)=\underline{c}^{T} \underline{l}(t)
\end{gathered}
$$

The convenience of the above state-space representation is that any standard statespace design techniques can be used for state-feedback control. However, simplicity and implementation considerations suggests the development of a predictive control law. In the past decade, several predictive control laws have been proposed, e.g. by Martin-Sanchez (1976), Richalet et al. (1978), Ydstie (1984), Clarke et al. (1987). Their major advantages are, simplicity of use, intuitive appeal, and easy handling of varying time-delay and non-minimum phase behaviour. From equation (2.54) we can write for the $d$-steps ahead output,

$$
y(t+d)=y(t)+\underline{c}^{T}[\underline{l}(t+d)-\underline{l}(t)]
$$


The recursive use of equation (2.51) gives for $d$ future sample points:

$$
\begin{aligned}
\underline{l}(t+1) & =A \underline{l}(t)+\underline{b} u(t) \\
\underline{l}(t+2) & =A \underline{l}(t+1)+\underline{b} u(t+1) \\
\cdots & =\cdots \\
\underline{l}(t+d) & =A \underline{l}(t+d-1)+\underline{b} u(t+d)
\end{aligned}
$$

Using the equations (3.59) in a continuing recursive substitution and assuming,

$$
u(t)=u(t+1)=\cdots=u(t+d-1)
$$

we get the $d$-steps ahead predictive expression:

$$
\underline{l}(t+d)=A^{d} \underline{l}(t)+\left(A^{d-1}+\cdots+I\right) \underline{b} u(t)
$$

Substituting equation (3.61) in equation (3.58) we finally get,

$$
y(t+d)=y(t)+\underline{k}^{T} \underline{l}(t)+\beta u(t)
$$

where,

$$
\begin{gathered}
\underline{k}^{T}=\underline{c}^{T}\left(A^{d}-I\right) \\
\beta=\underline{c}^{T}\left(A^{d-1}+\cdots+I\right) \underline{b}
\end{gathered}
$$

If $d>k_{d}$, where $k_{d}$ is the plant time-delay, the right-hand side of the above equation can be equated to the desired reference trajectory for the plant output. As in Richalet et al. (1978) we define a first order set-point reference trajectory based on the equations:

$$
\begin{gathered}
y_{r}(t+1)=\alpha y(t)+(1-\alpha) y_{s p} \\
y_{r}(t+i)=\alpha y_{r}(t+i-1)+(1-\alpha) y_{s p} \quad \mathrm{i}=2, \ldots, \mathrm{d}
\end{gathered}
$$


where $0<\alpha<1$ and $y_{s p}$ is the desired setpoint. By recursive substitution $y_{r}(t+d)$ can be written as:

$$
y_{r}(t+d)=\alpha^{d} y(t)+\left(1-\alpha^{d}\right) y_{s p}
$$

Setting $y(t+d)=y_{r}(t+d)$, and equating the right-hand parts of the equations (3.62) and (3.67) we can solve for the required control input $u(t)$ to get,

$$
u(t)=\left(y_{\tau}-y(t)-\underline{k}^{T} \underline{l}(t)\right) / \beta
$$

Remark 1: Let $k_{d}$ be the plant time-delay. For a minimum-phase plant with delay $k_{d}$, it is easy to show that $d>k_{d}$ and $\beta \neq 0$ are equivalent. However, for nonminimum phase systems, if one does not look sufficiently beyond the non-minimum phase behaviour, it is possible that $\beta=0$. In practice, one has to choose $d$ such that $\beta$ is of the same sign as the process static gain, and of sufficiently large amplitude. By definition,

$$
\beta=\underline{c}^{T}\left(A^{d-1}+\cdots+I\right) \underline{b}
$$

This shows that $\beta$ is the sum of the first $d$ sampling points of the impulse response of the plant.

Remark 2: The control law (3.68) can be expressed in velocity form. Equation (3.68) can be written as

$$
u(t)=\left(y_{r}-y(t)\right) / \beta-\underline{c}^{T} S(A \underline{l}(t)-A \underline{l}(t-1)-\underline{b} u(t-1)) / \beta
$$

Using the definition of $\beta$ and rearranging, one gets

$$
\Delta u(t)=\left(y_{r}-y(t)-\underline{d}^{T} \Delta \underline{l}(t)\right) \beta^{-1}
$$

where $S=\left(A^{d-1}+\cdots+I\right), \underline{d}^{\prime T}=\underline{c}^{T} S A, \Delta u(t)=u(t)-u(t-1)$, and $\Delta \underline{l}(t)=\underline{l}(t)-\underline{l}(t-1)$.

Remark 3: An alternate, receding control law as in Ydstie (1984) can also be derived. Then, at each step a control sequence $u(t), \ldots, u(t+d-1)$ that satisfies 
$y(t+d)=y_{r}(t+d)$ and that minimizes

$$
J_{d}=\sum_{i=0}^{d-1} u^{2}(t-i)
$$

is determined, but only $u(t)$ is implemented. Such a control sequence is given by,

$$
u(t+i)=\frac{\gamma_{i}\left[y_{r}(t+d)-y(t)-\underline{k}^{T} \underline{l}(t)\right]}{\gamma_{0}^{2}+\cdots+\gamma_{d-1}^{2}}
$$

where, $\gamma_{i}=\underline{c}^{T} A^{d-1-i} \underline{b}$, for $i=0, \ldots, d-1$.

The following proposition shows clearly the value of the derived predictive control law given by equation (3.68) with respect to cost indexing the control input.

Proposition 3 Let the system be described by (2.51) and (2.54). Then the control law, under the assumption (3.60), that satisfies $y(t+d)=y_{r}(t+d)$ is the same one as the control law that both satisfies $y(t+d)=y_{r}(t+d)$ and at each step minimizes the cost index:

$$
J_{0}=u(t)^{2}
$$

Proof : Consider $\left(y(t+d)-y_{r}(t+d)=0\right)$ as the constraint equation for the minimization of the cost function (3.73) then, the gradient of the Lagrangian function with respect to the input $u(t)$ is given by,

$$
2 u(t)-\mu\left(\gamma_{0}+\cdots+\gamma_{d-1}\right)=0
$$

where $\mu$ is the Lagrangian multiplier and the assumption (3.60) was used. Solving the above equation for $u(t)$ and substituting in (3.62) we get,

$$
\mu=\frac{2\left(y_{r}(t+d)-y(t)-\underline{k}^{T} \underline{l}(t)\right)}{\left(\gamma_{0}+\cdots+\gamma_{d-1}\right)^{2}}
$$

Now substituting (3.75) in (3.74) we derive for the control law $u(t)$ the same equation as (3.68). This is the reason why the equations (3.68) and (3.72) give similar simulation results. $\square$ 
The following Theorem proves, for the case of known fixed plant parameters, the convergence of the derived predictive control law.

Theorem 1 Let the system described by (2.51) and (2.54) be controlled by (3.68) where $\beta$ is nonzero, and assume $y_{s p}$ constant. Then there is some finite prediction horizon $d>k_{d}$, such that,

$$
\begin{aligned}
& \lim _{t \rightarrow \infty} y(t)=y_{s p} \\
& \lim _{t \rightarrow \infty} u(t)=u
\end{aligned}
$$

where $u$ is a constant. $\square$

Proof : The first part of the proof consists in proving the stability of the closed-loop system. For that the closed-loop system equations are derived and examined for the conditions of stability. The closed-loop system can be expressed by,

$$
\underline{l}(t+1)=\left(A-\underline{b} \underline{c}^{T}\left(1-\alpha^{d}\right) \beta^{-1}-\underline{b} \underline{k}^{T} \beta^{-1}\right) \underline{l}(t)+\underline{b}\left(1-\alpha^{d}\right) \beta^{-1} y_{s p}
$$

Using equation (3.63), one can write

$$
\underline{k}^{T}=\underline{c}^{T}\left(A^{d}-I\right)
$$

Substituting above in equation (3.76) and after some simple manipulations one obtains:

$$
\underline{l}(t+1)=\left(A+\underline{b} \underline{c}^{T} \alpha^{d} \beta^{-1}-\underline{b} \underline{c}^{T} \beta^{-1} A^{d}\right) \underline{l}(t)+\underline{b}\left(1-\alpha^{d}\right) \beta^{-1} y_{s p}
$$

For stability we examine the matrix,

$$
\left(A+\underline{b} \underline{c}^{T} \alpha^{d} \beta^{-1}-\underline{b} \underline{c}^{T} \beta^{-1} A^{d}\right)
$$


As long as the eigenvalues of the above matrix, for some value of $d$, are inside the unit disk in the $z$-plane then the closed-loop system is stable. $A$ is a stable matrix and its eigenvalues are always inside the stable region and not necessarily on the boundary zone. Therefore a proper choice of $d$ can be found that will relocate the eigenvalues of the expression (3.79) inside the stable region. The choice of $d$ has to be such as to keep the eigenvalues of the above matrix expression inside the unit disk. This condition must be met for stability.

However, for sufficiently large $d$, and while $\beta \neq 0$ the second term in expression (3.79) approaches zero because by definition $0<\alpha<1$. The third term in expression (3.79) also approaches zero, for sufficiently large $d$, because the square matrix $\mathrm{A}$ is a lower triangular and the eigenvalues of A appear along its main diagonal. It is straightforward then to show that the powers $A^{d}$ approach zero (Strang, 1976) because all the eigenvalues of A are less than one in modulus $\left(\left|\lambda_{i}(A)\right|<1\right)$. Finally, the first term in the expression (3.79) is always, by construction, a stable matrix and thus the closed-loop system is stable.

Determining the steady-state of the system is then trivial. From equation (3.77) and using $\left(A^{d-1}+\cdots+I\right)(I-A)=\left(I-A^{d}\right)$, one can write,

$$
\underline{k}^{T}=\underline{c}^{T} S(A-I)
$$

Premultiplying (3.76) by $\underline{c}^{T} S$ and using the above expression for $\underline{k}^{T}$, after some manipulations one obtains,

$$
\underline{c}^{T} S \underline{l}(t+1)=\underline{c}^{T}\left(S-\left(1-\alpha^{d}\right) I\right) \underline{l}(t)+\left(1-\alpha^{d}\right) y_{s p}
$$

From the above equation it is obvious that the steady-state is such that $y=y_{s p},\left(\right.$ because $\left.\lim _{t \rightarrow \infty}\|\underline{l}(t+1)-\underline{l}(t)\|=0\right)$. 
Remark 4: It is worthwhile to mention here that in practice, during the simulations, to increase $d$ to very large values was hardly ever needed for stability, because as long as $d>k_{d}$ then the number $N$ of the Laguerre filters can be always accordingly adjusted for a proper identification. And this is true for most of the process control loops encountered in practice. Of course if everything else fails then, in the above case, by increasing $d$ to some large value, stability can always be achieved.

Remark 5: Using $\left(A^{d-1}+\cdots+I\right)(I-A)=\left(I-A^{d}\right)$, one can write,

$$
\beta=\underline{c}^{T}\left(I-A^{d}\right)(I-A)^{-1} \underline{b}
$$

When $d$ is sufficiently large then, in the limit, we get,

$$
\beta=\underline{c}^{T}(I-A)^{-1} \underline{b}
$$

which is the static gain of the transfer function $G(z)$ of the state-space system $\left(A, \underline{b}, \underline{c}^{T}\right)$,

$$
\begin{gathered}
G(z)=\underline{c}^{T}(z I-A)^{-1} \underline{b} \\
\lim _{z \rightarrow 1} G(z)=\underline{c}^{T}(I-A)^{-1} \underline{b}
\end{gathered}
$$

In practice the static gain of the transfer function $G(s)$ can be computed roughly from,

$$
\lim _{s \rightarrow 0} G(s)=\lim _{s \rightarrow 0}\left(\sqrt{2 p} \sum_{i=1}^{N} \hat{c}_{i} \frac{(s-p)^{i-1}}{(s+p)^{i}}\right)=\frac{\sqrt{2 p}}{p} \sum_{i=1}^{N}(-1)^{i-1} \hat{c}_{i}
$$

where $\hat{c}_{i}$ 's are the identified Laguerre gains.

Remark 6: It is easy- to show that the closed-loop characteristic equation is

$$
\sum_{i=1}^{d} \underline{c}^{T} A^{i-1} \underline{b}+\underline{c}^{T}\left(A^{d}-\alpha^{d} I\right) \sum_{i=1}^{\infty} A^{i-1} q^{-i+1} \underline{b}=0
$$

When $d=k_{d}$ and $\alpha=0$, the 1.h.s. of the above equation is the impulse response of the plant. If it happens to be non-minimum phase, then the regulator is unstable. The best way to shift the regulator poles back inside the unit circle is to increase $d$. 


\subsection{The Self-tuning Controller}

\subsubsection{A Deterministic Explicit Self-Tuner}

As was suggested in Zervos and Dumont (1988a), it is straightforward to design an explicit deterministic adaptive control scheme based on the formulation described above. In principle, other orthonormal functions could be used, although Laguerre functions prove to be a good choice. The problem of identifying on-line the Laguerre spectrum gains (i.e. the parameter vector $\underline{c}$ ) is addressed by using the recursive least-squares (RLS) Identification method. Given $\left\{P(0) \geq \epsilon_{1} I>0, \hat{c}(0), \underline{l}(0), \lambda(0)\right\}$ then starting from $t=1$ we compute the parameter vector at any given sampling instant by using the following equations:

$$
\begin{gathered}
\hat{\hat{c}}(t)=\underline{\hat{c}}(t-1)+\frac{P(t-1) \underline{l}(t)}{\lambda(t)+\underline{l}^{T}(t) P(t-1) \underline{l}(t)}\left[y(t)-\underline{\hat{c}}^{T}(t-1) \underline{l}(t)\right] \\
P(t)=P(t-1)-\frac{P(t-1) \underline{l}(t) \underline{T}^{T}(t) P(t-1)}{\lambda(t)+\underline{\underline{l}}^{T}(t) P(t-1) \underline{l}(t)}
\end{gathered}
$$

where $\lambda(t)$ is the forgetting factor $(0<\lambda(t) \leq 1)$ and is used to discount away past data using exponentially decaying weights when tracking slowly time-varying parameters. It is also important in the computations above for the covariance matrix to factor $P(t)$ and update either the $\sqrt{P(t)}$ or the UD factors separately (Bierman, 1977; Ljung and Soderstrom, 1983). To include immunity against bias in the parameter identification such as those induced by offsets, the least-squares identification scheme uses increments of $\underline{l}(t), u(t)$ and $y(t)$ instead of full values. The control law (3.68) is then computed at every sampling instant.

Remark 1: Suppose,

$$
\lim _{t \rightarrow \infty} \inf \frac{1}{t} \sum_{i=1}^{t} \underline{l}(i) \underline{l}^{T}(i) \geq \epsilon_{2} I
$$


and $\lambda(t)=1$, then,

$$
\limsup _{t \rightarrow \infty}\|\hat{\underline{\hat{c}}}(t)\| \leq K
$$

for some small $\epsilon>0$ and some finite positive constant $\mathrm{K}$.

Proof: Assuming that $w(t)$ is a uniformly bounded sequence then by some algebraic manipulations (Ydstie, Kemna, Liu, 1987) we have,

$$
V(t)=V(t-1)-\frac{e^{2}(t)}{\left(1+\underline{l}^{T}(t) P(t-1) \underline{l}(t)\right)}+w^{2}(t)
$$

where $e(t)$ is the prediction error defined by,

$$
e(t)=y(t)-\underline{\underline{l}}^{T}(t) \hat{\hat{c}}(t-1)
$$

and $V(t)$ is a scalar Lyapunov function given by,

$$
V(t)=(\underline{c}-\underline{\hat{c}}(t))^{T} P^{-1}(t)(\underline{c}-\underline{\hat{c}}(t))
$$

By summing up we get,

$$
\frac{1}{t} V(t) \leq \frac{1}{t} \sum_{i=1}^{t} w^{2}(i)+0(1 / t)
$$

where the second term in the r.h.s. is due to the effect of non-zero initial conditions and decays as $1 / t$. Now using the matrix inversion lemma we can write,

$$
\begin{aligned}
P^{-1}(t) & =P^{-1}(t-1)+\underline{l}(t) \underline{l}^{T}(t) \\
& =\sum_{i=1}^{t} \underline{l}(i) \underline{T}^{T}(i)+P^{-1}(0)
\end{aligned}
$$

and from equations (3.90), (3.89) and from the assumption (3.84) we can get,

$$
\limsup _{t \rightarrow \infty}\|\underline{c}-\hat{s}(t)\|^{2} \leq \limsup _{t \rightarrow \infty} \frac{1}{t} \sum_{i=1}^{t} w^{2}(i) / \epsilon
$$

and the result of equation (3.85) follows since $w(i)$ was assumed to be a uniformly bounded sequence. The condition on the vector $\underline{l}$ is referred to as the persistency 
excitation condition. This is rarely satisfied in practice so in order to avoid parameter drift problem that may occur when not enough information is fed to the estimator one can turn off the estimation when there is not enough process excitation. This can be achieved by using a dead zone $\Delta$ with the property,

$$
\left\{\begin{array}{l}
\hat{c}(t)=\hat{c}(t-1) \\
P(t)=P(t-1)
\end{array}\right\} \text { if } \quad|e(t)| \leq \Delta, \quad \Delta>0
$$

In that case the estimation algorithm is turned off when the prediction error is small. The proper size for $\Delta$ is determined by the measurement noise in the system. The estimation should also be turned off if either the input or output signals hit constraints.

Remark 2: Assume $\lambda(t)=1$ and define,

$$
x(t)=1+\underline{l}^{T}(t) P(t-1) \underline{l}(t)
$$

If $q$ satisfies,

$$
q^{\prime}=\min _{\underline{\underline{c}}} \frac{1}{t} \sum_{i=1}^{t}\left(y(i)-\underline{c}^{T} \underline{l}(i)\right)^{2}
$$

Then,

$$
\limsup _{t \rightarrow \infty} \frac{1}{t} \sum_{i=1}^{t} \frac{e^{2}(i)}{x(i)} \leq q
$$

Remark 3: If the forgetting factor is less than unity then a similar result as above holds. Suppose $0<\lambda(t) \leq \lambda<1$, then if

$$
\sum_{i=t-m}^{t} \underline{l}(i) \underline{I}^{T}(i) \geq \epsilon I>0 \quad, \quad \text { for all } \quad t>m
$$

where $m$ is a finite positive integer, then,

$$
\limsup _{t \rightarrow \infty}|\hat{\hat{c}}(t)| \leq K<\infty
$$

and;

$$
\limsup _{t \rightarrow \infty} \frac{1}{t} \sum_{i=1}^{t} \frac{e^{2}(i)}{x(i)} \leq q
$$


The Remarks 2,3 , show the boundness of the normalized parameter estimation error for $0<\dot{\lambda} \leq 1$ and their proof techniques follow along the same lines as those presented in Ydstie, et al. (1987).

The adaptive control scheme as described so far is globally convergent, as shown by the following theorem.

Theorem 2 Assume that the plant is described by $y(t)=\underline{c}_{0}^{T} \underline{l}(t)$, then provided that the projection or least-squares algorithm (3.82), (3.83) is used to find $\underline{\hat{c}}(t)$, that $\operatorname{dim} \underline{\hat{c}}=$ $\operatorname{dim} \underline{c}_{0}$, and that $\beta \neq 0$, then the indirect adaptive control scheme described above is globally convergent in the sense that

i) $\{u(t)\},\{y(t)\}$ are bounded for all time $t$.

ii) $\lim _{t \rightarrow \infty}\left[y(t)-y_{s p}(t)\right]=0$

Proof: Using standard arguments (Samson and Fuchs, 1981; Goodwin and Sin, 1984), the parameter adaptation scheme can be shown to be such that:

1. $\{\underline{\hat{c}}(t)\}$ is bounded,

2. $\lim _{t \rightarrow \infty}\|\underline{\hat{c}}(t)-\underline{\hat{c}}(t-1)\|=0$,

3. There exist non-negative sequences $\{\xi(t)\},\{\psi(t)\}$ that converge to zero and such that:

$$
|\hat{y}(t)-y(t)| \leq \xi(t)\|\underline{l}(t)\|+\psi(t)
$$

Assuming, for simplicity purposes that the parameter $\alpha$ in equation (3.67) is zero, one can write the closed-loop system as:

$$
\underline{l}(t+1)=\left(A-\underline{b} \underline{\hat{c}}^{T} A^{d} \hat{\beta}^{-1}\right) \underline{l}(t)+\underline{b} \hat{\beta}^{-1}\left(y_{s p}+\hat{y}(t)-y(t)\right)
$$


or

$$
\underline{l}(t+1)=F(t) \underline{l}(t)+\underline{v}(t)
$$

From Theorem 1, it is easy to show there for a proper choice of $d$ the free system

$$
\underline{l}(t+1)=F(t) \underline{l}(t)
$$

is exponentially stable. With

$$
\|\underline{v}(t)\| \leq\left.|\|\underline{b}\|| \hat{\beta}\right|^{-1}\left(\left|y_{o p}\right|+|\hat{y}(t)-y(t)|\right)
$$

if for simplicity $y_{s p}=0$, then using the properties of the adaptation scheme (equation (3.99)), we can write

$$
\|\underline{v}(t)\| \leq \gamma(t)\|\underline{l}(t)\|+\delta(t)
$$

with

$$
\begin{aligned}
& \gamma(t)=\|\underline{b}\| \cdot \xi(t)|\hat{\beta}|^{-1} \\
& \delta(t)=\|\underline{b}\| \psi(t)|\hat{\beta}|^{-1}
\end{aligned}
$$

Because both $\{\gamma(t)\}$ and $\{\delta(t)\}$ converge to zero, then from Theorem 2.1 in Payne (1987), $\{\underline{l}(t)\}$ and $\{\underline{v}(t)\}$ are bounded and converge to zero. When $y_{a p} \neq 0,\{\underline{l}(t)\}$ and $\{\underline{v}(t)\}$ are bounded and it is trivial to show that they converge such that:

$$
\begin{gathered}
\lim _{t \rightarrow \infty} \underline{v}(t)=\underline{b} \hat{\beta}^{-1} y_{s p} \\
\lim _{t \rightarrow \infty} \underline{\hat{c}}^{T}(t) \underline{l}(t)=y_{o p}
\end{gathered}
$$




\subsubsection{A Deterministic Implicit Self-tuner}

A deterministic implicit self-tuner can also be derived using the Laguerre orthonormal set. The controller parameters are then estimated instead of the model ones. The identification would involve the model,

$$
y(t)-y(t-d)=\underline{\hat{k}}^{T} \underline{l}(t-d)+\hat{\beta}_{d} u(t-d)+\ldots+\hat{\beta}_{1} u(t-1)
$$

where the vector parameter gain $\underline{\hat{k}}$ and the input coeffients $\hat{\beta}_{i}$ 's are identified on line. The control law then is,

$$
u(t)=\left(y_{r}-y(t)-\underline{\hat{k}}^{T} \underline{l}(t)\right) \hat{\beta}_{t o t}^{-1}
$$

where $\hat{\beta}_{t o t}=\hat{\beta}_{1}+\ldots+\hat{\beta}_{d}$. An example follows showing the applicability of the derived implicit scheme.

\subsection{Simulation results}

The described adaptive self-tuning schemes based on the Laguerre series representation have been tested extensively in simulations. Some examples showing their potential applicability and illustrating their practicality follow. The simulations were performed on a MicroVAX-II/VMS supermicro computer using Fortran-77 and/or ACSL (Advanced Continuous Simulation Language).

Example 3.1: Consider the closed-loop system $H\left(q^{-1}\right)$ of Figure 3.7

$$
H\left(q^{-1}\right)=\frac{G_{c}\left(q^{-1}\right) G\left(q^{-1}\right)}{1+G_{c}\left(q^{-1}\right) G\left(q^{-1}\right)}
$$

with,

$$
\begin{gathered}
G_{c}\left(q^{-1}\right)=K_{c} \frac{1+0.6048 q^{-1}}{1-0.3697 q^{-1}} \quad \text { where } K_{c}=0.3397 \\
G\left(q^{-1}\right)=\frac{0.0193\left(1+0.0672 q^{-1}\right)}{1+1.9048 q^{-1}-0.9048 q^{-2}} q^{-k_{d}}
\end{gathered}
$$




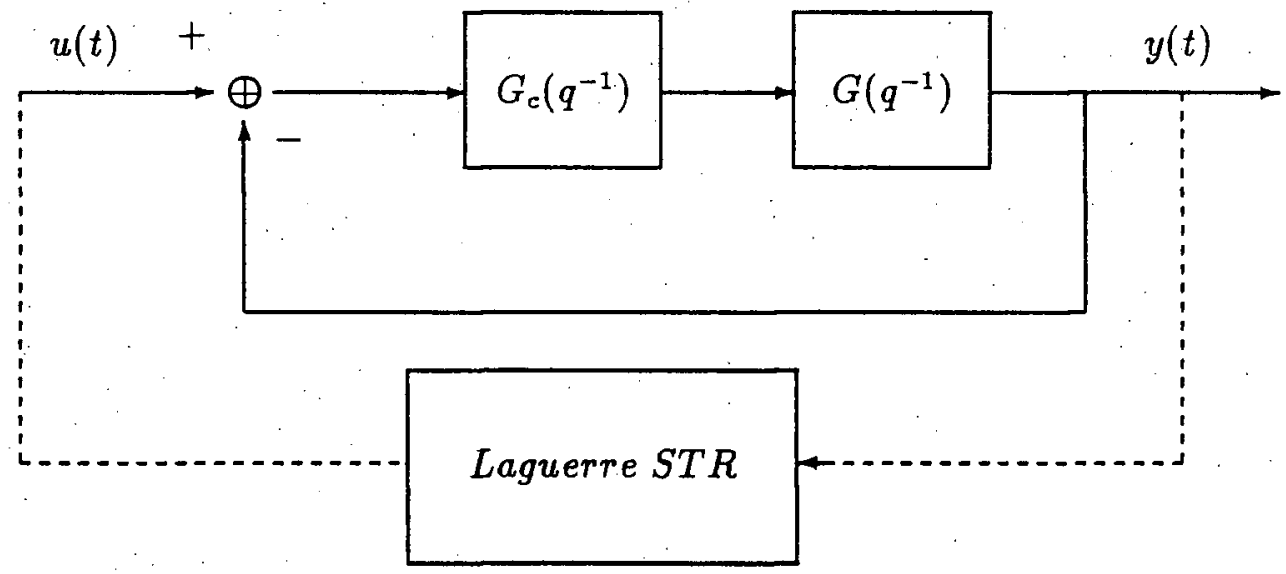

Figure 3.7: System to be controlled in Example 3.1. 
Figure 3.8 shows the step responses of $H\left(q^{-1}\right)$ (i.e from $u$ to $y$ ) with $k_{d}=1$, with and without noise. Note the oscillatory nature of the response as well as the load disturbance of amplitude 0.2 from time $t=240$. For the noisy response, an FIR noise filter with unity $C$ polynomial has been added to corrupt the plant output. The Laguerre adaptive controller is used to control the system $H\left(q^{-1}\right)$ as in Figure 3.7, with $N=10$ Laguerre filters and $p=0.1$. Initial parameter estimates are zero. The initial covariance matrix is $100 \times I$, and the forgetting factor is 1 . Figure 3.9 shows the behaviour of the adaptive controller with a prediction horizon $d=2$ and the driver block filter time constant $\alpha=0.5$, both with and without noise. The start-up transient has a very small amplitude. Both the response to the setpoint change and the load disturbance rejection are excellent. As expected from Theorem 1 there is no steadystate offset. Figure 3.10 shows runs when the dead time $k_{d}$ in $G\left(q^{-1}\right)$ is increased from 1 to 3 sampling intervals, both with and without noise. The scheme is exactly the same as in Figure 3.9 except for $d=4$ and $\alpha=0.7$. Again, a load disturbance of amplitude 0.2 was applied from time $t=240$. Further simulations have shown the good performance of that scheme for the regulator problem as well.

Example 3.2: This time, we consider the non-minimum phase plant described by

$$
y(t)-0.7 y(t-1)=u(t-1)+2 . u(t-2)
$$

This plant was used by Clarke (1984) to demonstrate a pole placement self-tuning controller. Here, we shall use the same sequence of setpoint changes and the same commissioning period as in Clarke (1984). Figure 3.11 shows the output of the above plant tracking a square-wave setpoint when it is under Laguerre self-tuning control, started with zero initial parameter estimates, and set with $d=2$ and $\alpha=0.7$, i.e. the same conditions as in Figure 3.9. The performance is very good, and compares well with that obtained by Clarke with a pole-placement self-tuner based on a model of 

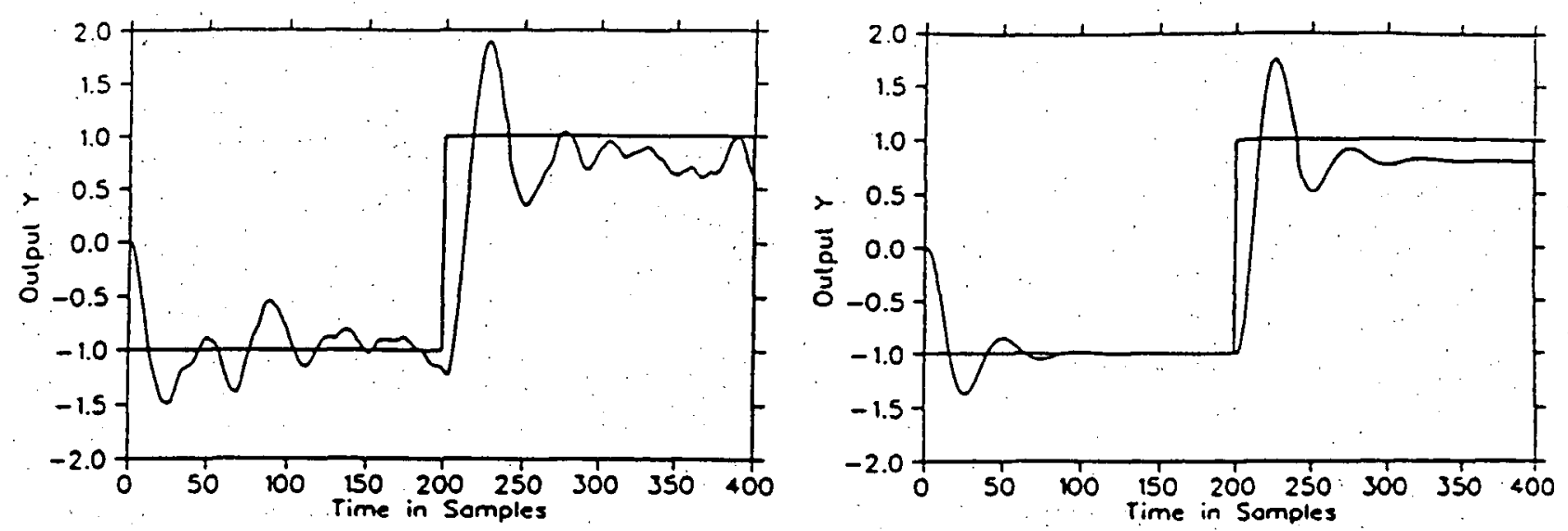

Figure 3.8: Example 3.1: Output responses of $H\left(q^{-1}\right)$, (a) with and (b) without noise, $k_{d}=1$.
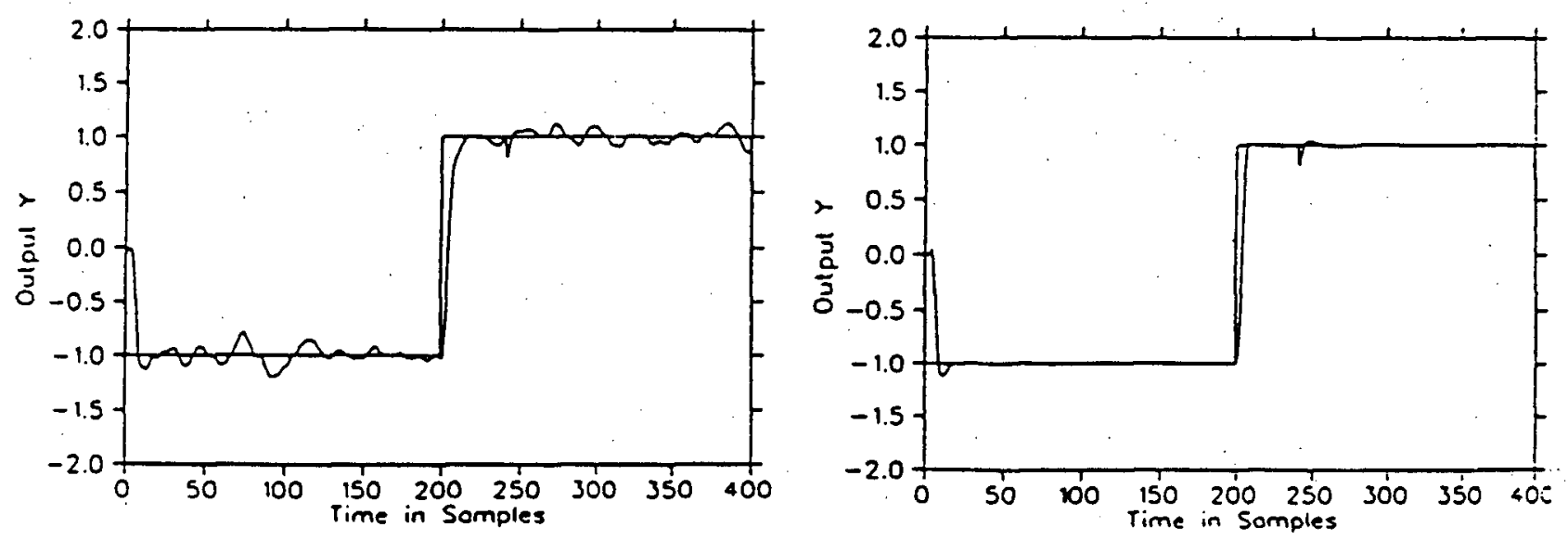

Figure 3.9: Example 3.1: Output responses, (a) with and (b) without noise, when $H\left(q^{-1}\right)$ is under Laguerre self-tuning control, $k_{d}=1, d=2$. 

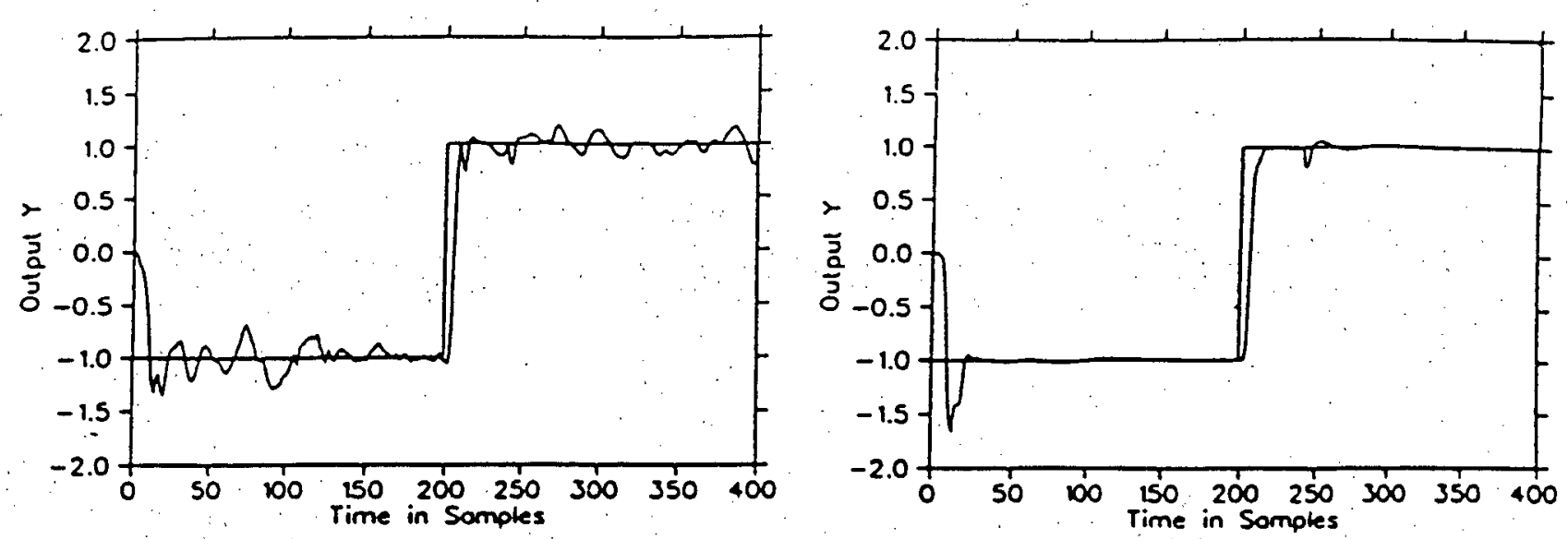

Figure 3.10: Example 3.1: Output responses, (a) with and (b) without noise, when $H\left(q^{-1}\right)$ is under Laguerre self-tuning control, $k_{d}=3, d=4$.

exactly the same structure as the plant. However, as noted by Clarke, his scheme does not behave well in the presence of unmodelled dynamics. Indeed when applied to the plant,

$$
y(t)-1.7 y(t-1)+0.72 y(t-2)=0.1 u(t-1)+0.2 u(t-2)
$$

the Clarke pole-placement self-tuner based on a first-order model eventually destabilizes the plant. The Laguerre self-tuner was used on this 2 nd-order plant with exactly the same design parameters as with the 1st-order plant. Results presented on Figure 3.12 show the excellent behaviour of this scheme. Note that Figures $3.9,3.11$ and 3.12 . have all been obtained with the same Laguerre self-tuning scheme and the same initial set-up parameters, although the three plants are all different. This is an indication of the robustness of the Laguerre self-tuner.

Example 3.3: The present method is limited to stable plants. It is thus interesting to see what happens when applied to a plant containing an integrator, a common 


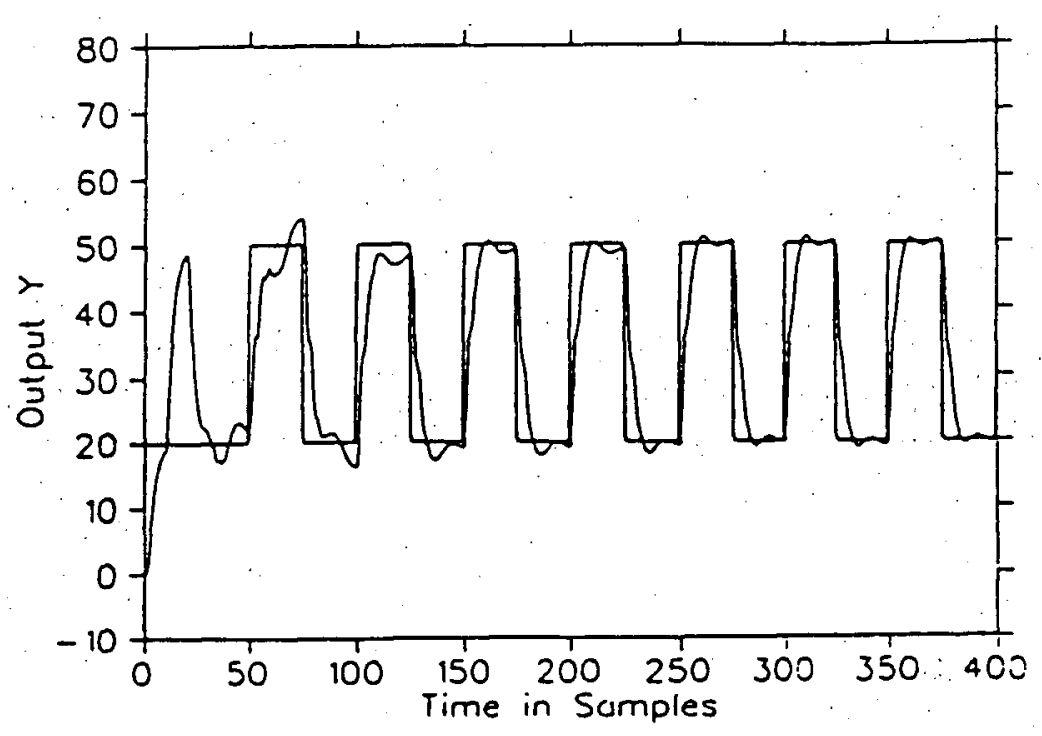

Figure 3.11: Example 3.2: System response under Laguerre self-tuning control, $1^{a t}$ order plant.

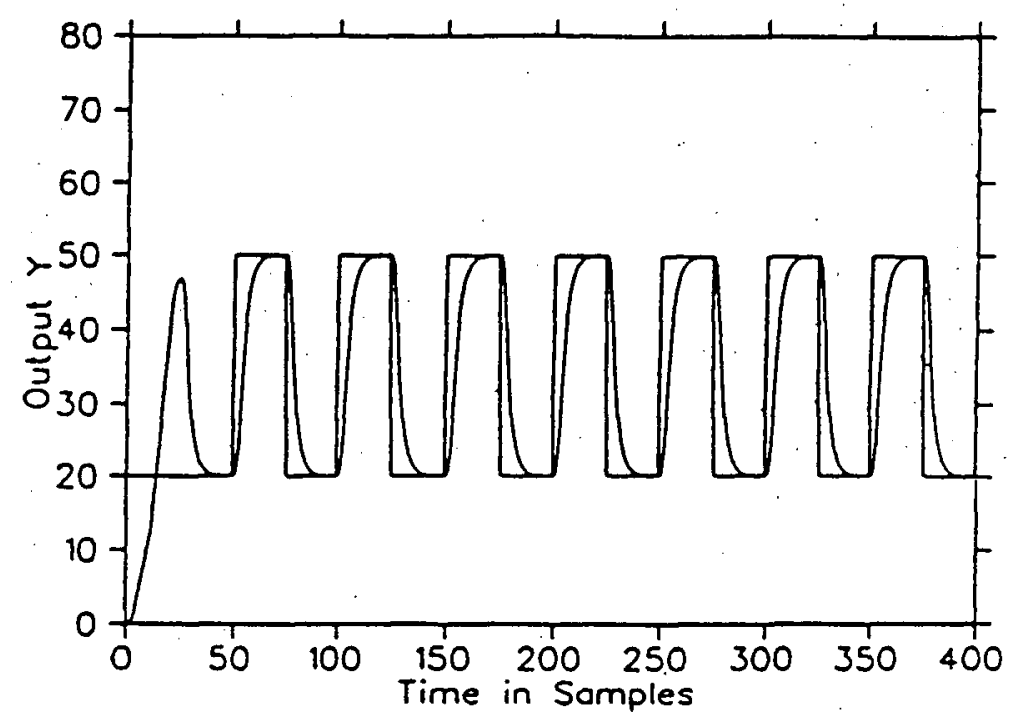

Figure 3.12: Example 3.2: System response under Laguerre self-tuning control, $2^{\text {nd }}$ order plant. 
occurrence in process control: A logical way to represent a plant with integrator is,

$$
\begin{gathered}
\underline{l}(t+1)=A \underline{l}(t)+\underline{b} u(t) \\
\Delta y(t)=\underline{c}^{T} \underline{l}(t)
\end{gathered}
$$

We now assume,

$$
u(t) \neq 0, \quad u(t+1)=\cdots=u(t+d-1)=0 .
$$

The $d$-step ahead predictor is then,

$$
\begin{gathered}
y(t+d)=y(t)+\Delta y(t+1)+\cdots+\Delta y(t+d) \\
y(t+d)=y(t)+\underline{d}^{\prime} \underline{l}(t)+\beta u(t)
\end{gathered}
$$

The control law is then (see equation 3.70 for definition of $\underline{d}^{\prime}$ ),

$$
\Delta u(t)=\left(\dot{y}_{\tau}(t+d)-y(t)-\underline{d^{\prime}} \underline{l}(t)\right) \beta^{-1}
$$

Compare with (3.68) and (3.70). Now, consider the plant described by,

$$
y(k)=-1.9048 y(k-1)+0.9048 y(k-2)+0.5(u(k-1)+0.0672 u(k-2))
$$

This plant contains an integrator. Figure 3.13 shows a simulation run when the plant is under Laguerre self-tuning control and the output is tracking a square-wave setpoint. The Laguerre parameters used were, $N=8, p=0.25$ and $d=3$. Good simulation results were also obtained in the range $0.05<p<0.5$. Note that a load disturbance was introduced from $t=240$ to $t=260$.

Example 3.4: The dynamics of an existing two-link manipulator were simulated using Paul's equations (Paul, 1981) and ACSL (Advanced Continuous Simulation Language) on a DEC VAX-11/750 computer. The manipulator has two degrees of freedom 


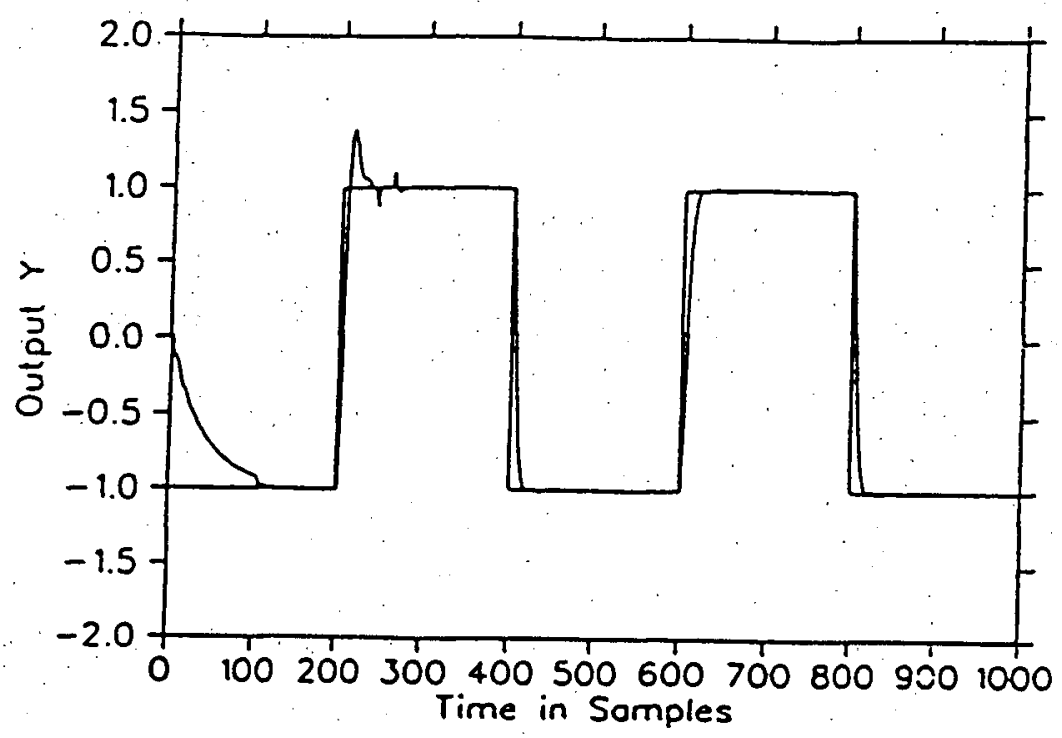

Figure 3.13: Example 3.3: Laguerre self-tuning Control of a system with integrator.

on the horizontal plane. Each link has a mass of $1 \mathrm{Kg}$ and length of $0.5 \mathrm{~m}$. All forces due to gravity, coupling inertia, centripetal acceleration and Coriolis acceleration were included in the dynamics of the arm. Two single-input/output Laguerre self-tuning controllers were implemented, one for each link. Each self-tuner controls the joint angle by the manipulation of the motor armature drive voltage. Several reference trajectories were implemented to test the reliablity of the proposed self-tuning algorithm including desired angles of $90^{\circ}$ and $270^{\circ}$, and circular and linear trajectories. Other tests involved increasing the mass at the end point of the second link (equivalent of picking up a payload) part way into the trajectory, and adding armature inductance to test the ability of the controllers to deal with an unexpected pole in the system.

The controllers generally performed quite satisfactorily. The initial values and the parameter settings used for both controllers, were : $N=8, p=2$, sampling time $T=0.02 s, d=4, \alpha=0.2$ for the first 100 points and 0.7 subsequently. The parameter estimates were initialized to zero and the controllers outputs were limited to 
$\pm 20 \mathrm{Volts}$, the maximum voltage the motors can sustain. The incremental version of the controllers was used. All the initial manipulator angles were set to $0^{\circ}$. For the particular experiment regarding the on-line increase of the payload, at $t=4.0 \mathrm{~s}$ during a clockwise circular trajectory centered at $(0.5,0.0)$ of radius 0.15 , the mass at the end of the second link has been increased from 0 to $10 \mathrm{Kg}$ to simulate the pickup of a load. The arm was still capable of tracking the reference circle (as seen from Figure 3.14).

In another experiment we increased the armature inductance in both motors from $100 \mu \mathrm{H}$ up to $100 \mathrm{mH}$ to see if the unexpected poles could be handled without having to increase $N$ or change $p$. For values of the inductance $L_{m}$ less than $10 m H$ the controllers continued to track well (Figure 3.15) while at $100 \mathrm{mH}$ the 2-link manipulator system became eventually unstable. However by increasing the prediction horizon $d$ to 12 the system was capable again of giving acceptable results (Figure 3.16) with a penalty on the rise time. A side benefit is the reduced overall overshoot.

The results showed that tracking circles, lines and square waves proved to be no problem for the controllers. The only problems were the observed overshoot and some slight torque and controller chatter. Torque chatter was not eliminated although the armature inductor acted like a low pass filter and reduced the amplitude and frequency of the chatter. As far as the overshoot is concerned the increase of the prediction horizon $d$ contributed toward reducing it.

Example 3.5: Consider the same plant as in the Example 3.1, whose block diagram appears in Figure 3.7. The delay of the plant was set to $k_{d}=3$. Everything else remains the same. An implicit self-tuning scheme was now applied in the place of the Laguerreself-tuner block. The parameters used were, $N=10, p=0.3, d=4, \alpha=0.7$. The response of the plant's output following a square-wave setpoint sequence is shown in Figure 3.17, and the controller's output is shown in Figure 3.18. 


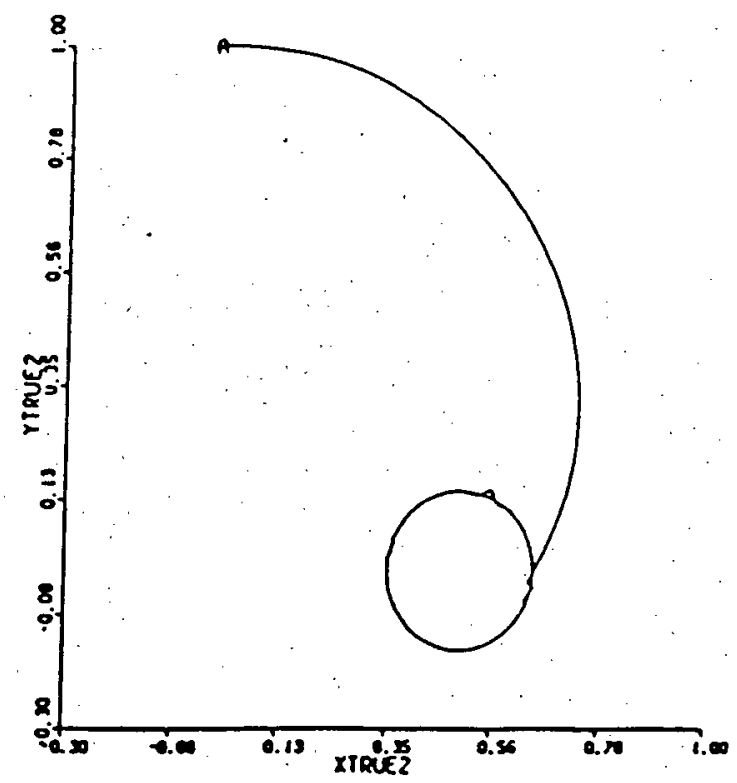

Figure 3.14: Example 3.4: Manipulator Arm tracking a circular trajectory. Payload increase, $m_{2}=10 \mathrm{Kgr}$.

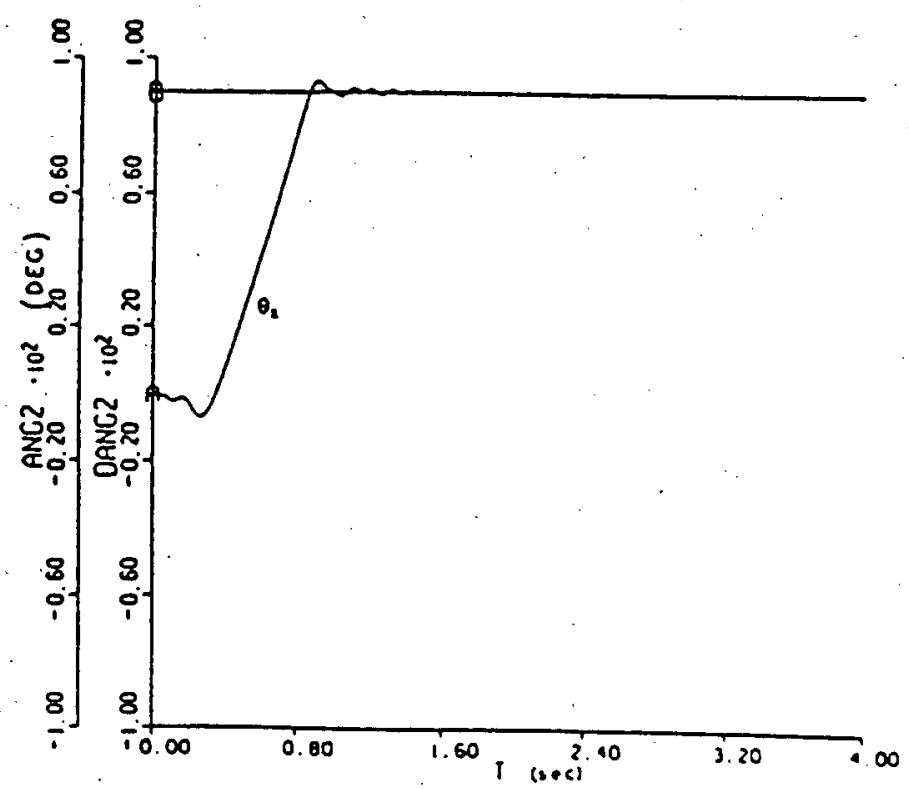

Figure 3.15: Example 3.4: Manipulator Arm tracking a step trajectory. Response of $2^{\text {nd }}$ joint angle. Inductance increase. $d=4$. 


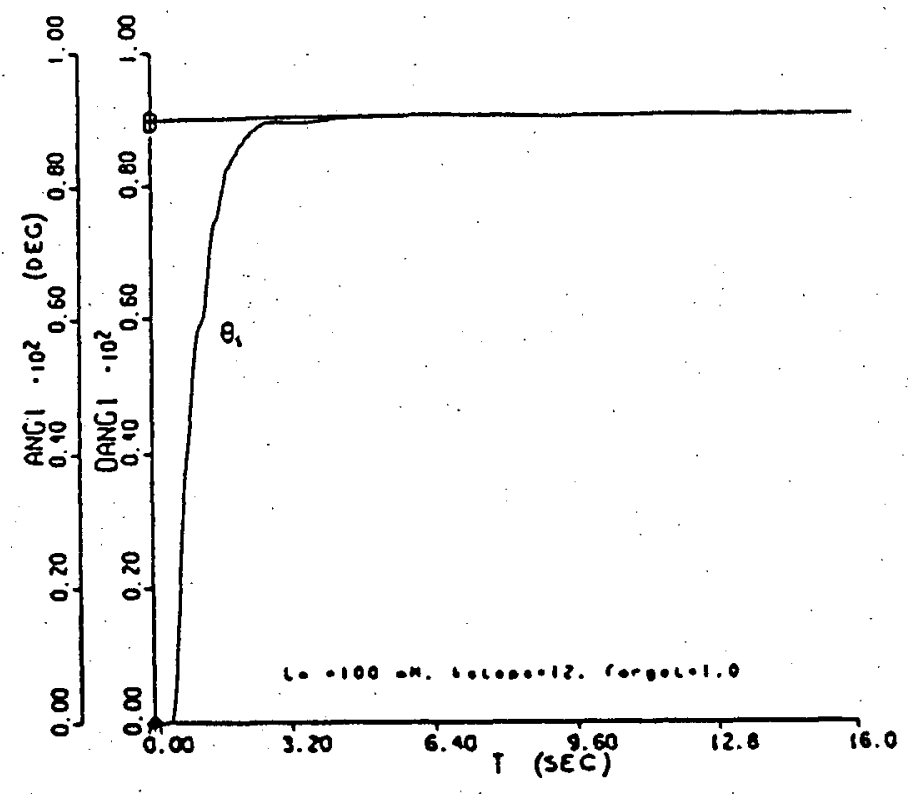

Figure 3.16: Example 3.4: Manipulator Arm tracking a step trajectory. Response of $1^{\text {ot }}$ joint angle. Inductance increase. $d=12$.

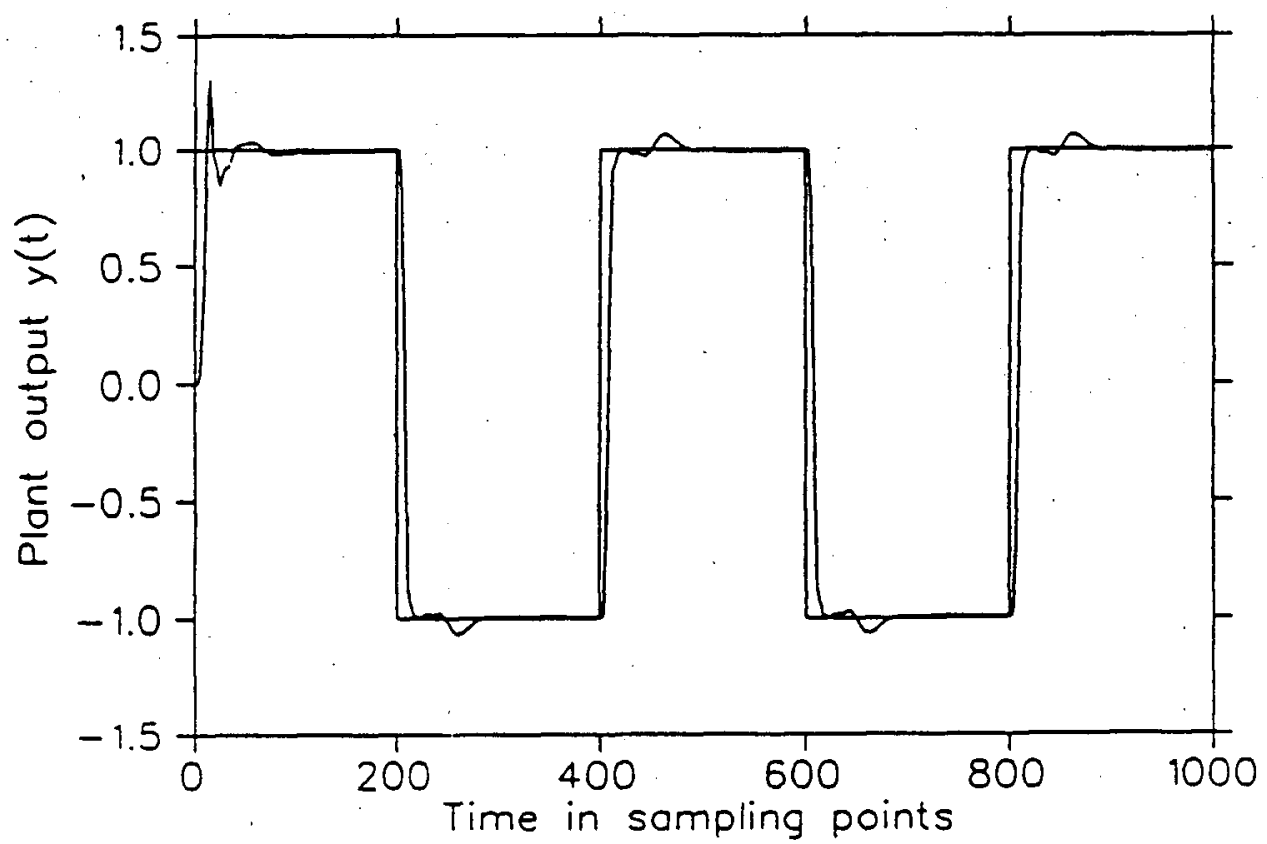

Figure 3.17: Example 3.5: Plant output following a square-wave setpoint when an implicit self-tuner was employed. 


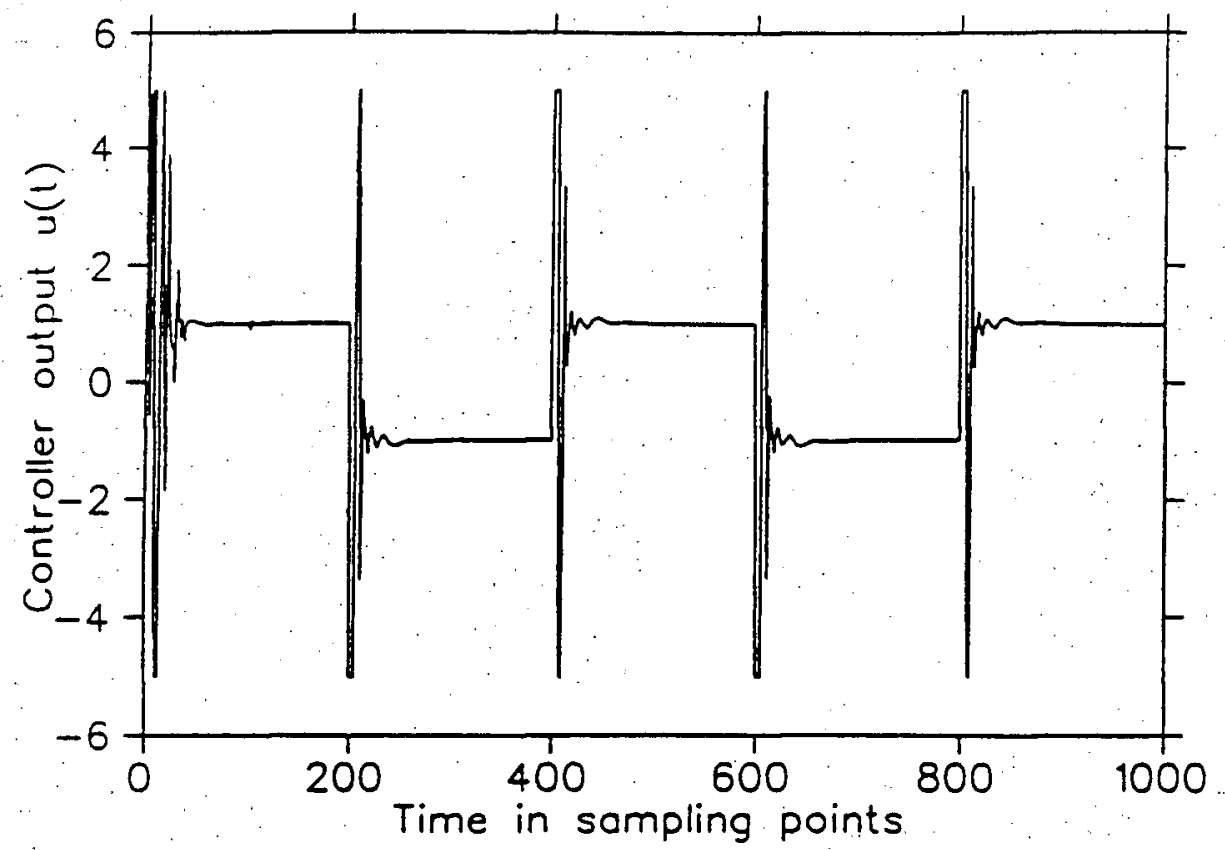

Figure 3.18: Example 3.5: Controller's output for the implicit self-tuning scheme.

\subsection{Robustness issues}

Because this method does not rely on a predefined model structure with a fixed number of poles and zeros, and because it does not separate the delay from the dynamics, we expect it to be more robust than the schemes based on transfer function models. The simple analysis and the examples that follow seem to indicate that this is the case.

Let the true deterministic plant be represented by the state-space equations:

$$
\begin{gathered}
\underline{x}_{t+1}=A_{0} \underline{x}_{t}+\underline{b}_{0} u_{t} \\
y_{t}=\underline{c}_{0}^{T} \underline{x}_{t}
\end{gathered}
$$

And let the Laguerre ladder network model of the above plant be represented by the equations:

$$
\begin{gathered}
\underline{\underline{l}}_{t+1}=A \underline{l}_{t}+\underline{b}_{t} \\
\hat{y}_{t}=\underline{\hat{c}}^{T} \underline{l}_{t}
\end{gathered}
$$


Where $\underline{x}$ and $\underline{l}$ are the state vectors respectively, not necessarily of the same order.

Let the system be under self-tuning control using the (non-linear w.r.t. the identified parameter vector) predictive control law derived in section 3.1 , equation (3.68), i.e.

$$
u_{t}=\left(y_{r}-y_{t}\right) \hat{\beta}^{-1}-\underline{\hat{k}}^{T} \underline{l}_{t} \hat{\beta}^{-1}
$$

Where $y_{r}$ is the $d$-steps-ahead $\left(d \geq k_{d}\right)$ pre-defined reference trajectory as given by,

$$
y_{r}=\alpha^{d} y_{t}+\left(1-\alpha^{d}\right) y_{s p}
$$

Where, $0 \leq \alpha<1$. Using the above control law (equation (3.119) and substituting for it in equations (3.115), (3.117), we get the following set of equations respectively:

$$
\begin{gathered}
\underline{x}_{t+1}=A_{0} \underline{x}_{t}+\underline{b}_{0}\left(\left(y_{r}-y_{t}\right) \hat{\beta}^{-1}-\underline{\hat{k}}^{T} \underline{l}_{t} \hat{\beta}^{-1}\right) \\
\underline{l}_{t+1}=A \underline{l}_{t}+\underline{b}\left(\left(y_{r}-y_{t}\right) \hat{\beta}^{-1}-\underline{\hat{k}}^{T} \underline{l}_{t} \hat{\beta}^{-1}\right)
\end{gathered}
$$

Substituting $y_{t}$ from equation (3.116) and $y_{\tau}$ from equation (3.120), the above set of equations can be written in a form to describe the closed-loop system as:

$$
\left[\begin{array}{c}
\underline{x}_{t+1} \\
\underline{l}_{t+1}
\end{array}\right]=\left[\begin{array}{cc}
A_{0}-\left(1-\alpha^{d}\right) \hat{\beta}^{-1} \underline{b}_{0} \underline{c}_{0}^{T} & -\underline{b}_{0} \underline{\hat{k}}^{T} \hat{\beta}^{-1} \\
-\left(1-\alpha^{d}\right) \hat{\beta}^{-1} \underline{b}_{\underline{0}}^{T} & A-\underline{b} \underline{\hat{k}} \hat{\beta}^{-1}
\end{array}\right]\left[\begin{array}{c}
\underline{x}_{t} \\
\underline{l}_{t}
\end{array}\right]+\left[\begin{array}{c}
\underline{b}_{0} \\
\underline{b}
\end{array}\right]\left(1-\alpha^{d}\right) \hat{\beta}^{-1} y_{s p}
$$

For stability, the $A$-matrix of the above closed-loop state-space description must have all of its eigenvalues inside the unit disk. If not, the closed-loop system will be unstable. Let us further assume that the output model mismatch between the true plant and the identified (modelled) one can be described by some arbitrary function, say $\zeta(t)$, which has the property that it stays always well bounded for all $t$, i.e. $|\zeta(t)| \leq Z<\infty$ where $Z$ is a positive real number $\left(Z \in \Re^{+}\right)$. The signal $\zeta(t)$ can be any bounded deterministic or stochastic signal, e.g. measurement noise, sensor drifts, modelling residual, etc. Then we can write,

$$
y_{t}=\hat{y}_{t}+\zeta_{t} \quad, \text { or, } \quad\left|y_{t}-\hat{y}_{t}\right|=\left|\zeta_{t}\right| \leq Z
$$


Expressing $y_{t}$ in terms of $\hat{y}_{t}$ by using equations (3.124) and (3.118) then the closed-loop state-space description given in (3.123) can be further written as,

$$
\left[\begin{array}{c}
\underline{x}_{t+1} \\
\vdots \\
\underline{l}_{t+1}
\end{array}\right]=\left[\begin{array}{cc}
A_{0} & -\underline{b}_{0}\left(\left(1-\alpha^{d}\right) \underline{\hat{c}}^{T}+\underline{\hat{k}}^{T}\right) \hat{\beta}^{-1} \\
\emptyset & A-\underline{b}\left(\left(1-\alpha^{d}\right) \underline{\hat{c}}^{T}+\underline{\hat{k}}^{T}\right) \hat{\beta}^{-1}
\end{array}\right]\left[\begin{array}{c}
\underline{x}_{t} \\
\underline{l}_{t}
\end{array}\right]+\left[\begin{array}{c}
\underline{b}_{0} \\
\underline{b}
\end{array}\right] \hat{\beta}^{-1}\left(1-\alpha^{d}\right)\left(y_{s p}-\zeta_{t}\right)
$$

Where $\emptyset$ is an all-zero matrix of the appropriate dimensions. Now let us define the $A$-matrix in equation (3.125) as,

$$
\mathbf{A}_{\mathrm{cl}}=\left[\begin{array}{cc}
A_{0} & -\underline{b}_{0}\left(\left(1-\alpha^{d}\right) \underline{\hat{c}}^{T}+\underline{\hat{k}}^{T}\right) \hat{\beta}^{-1} \\
\emptyset & A-\underline{b}\left(\left(1-\alpha^{d}\right) \underline{\hat{c}}^{T}+\underline{\hat{k}}^{T}\right) \hat{\beta}^{-1}
\end{array}\right]
$$

We can now present the following theorem concerning the stability of the closed-loop system.

Theorem 3 Let a stable discrete-time system be represented by the set of state-space equations (3.115),(3.116) and let it be sampled every $T$ secs and be under predictive control law with $d \geq k_{d}$ as described in Section 3.1 (i.e. equation (3.119)). Assume that the output model mismatch between the true plant and the identified (modelled) one can be expressed by any bounded arbitrary deterministic or stochastic signal $\zeta(t)$ such that $|\zeta(t)| \leq Z<\infty$. Then there is some prediction horizon d such that the closed-loop adaptive system remains always stable.

Proof: Is is easy to show the validity of the above statement when condition (3.124) is true by evaluating the $\mathbf{A}_{\mathbf{c l}}$ expression given by equation (3.126): Under the condition of equation (3.124) the closed-loop system can be put in state-space description as shown before by the set of expressions in equation (3.125). The stability of the overall system is then determined by the upper block-triangular matrix $\mathbf{A}_{\mathbf{c l}}$ as given by equation (3.126). 
Because of its special structure the eigenvalues of the matrix $\mathbf{A}_{\mathbf{c l}}$ are just the eigenvalues of the matrix $A_{0}$ plus the eigenvalues of the matrix :

$$
\mathbf{A}_{\mathbf{c l}}{ }^{(2,2)}=A-\underline{b}\left(\left(1-\alpha^{d}\right) \underline{\hat{c}}^{T}+\underline{\hat{k}}^{T}\right) \hat{\beta}^{-1}
$$

Now since the true plant under study was assumed to be stable then the eigenvalues of the matrix $A_{0}$ are always inside the unit disk. Besides, standard arguments from the proofs of Theorem 1 and Theorem 2 indicate that the matrix $\mathbf{A}_{\mathbf{c l}}{ }^{(2,2)}$ is also to be stable, for a proper choice of $d$, and have all its eigenvalues less than unity in modulus (compare equation (3.76) with (3.127)). As a result the closed-loop system remains always stable.

An illustrative example follows that makes use of the stability study described above.

Example 3.6: Let the continuous-time stable plant (Rohrs et al., 1985) of the form:

$$
G(s)=\frac{2}{(s+1)} \frac{229}{\left(s^{2}+30 s+229\right)}
$$

be sampled very $T$ secs. The input-output data is recursively used at every sampling step (RLS) to derive a discrete model of the plant in terms of a Laguerre orthonormal series as described in Section 2.3, and a predictive control law is then computed,as described in Section 3.1, which is applied to the plant on-line thus forming a closedloop self-tuning system.

First let the sampling time be $0.1 \mathrm{sec}$ and the reference input be $\sin (\omega t)$ with $\omega=$ $1 \mathrm{rad} / \mathrm{s}$. Underestimating the plant order and assuming that it is of a first order, only one Laguerre gain is estimated during the identification $(N=1, T=0.1, p=0.5, d=$ $2, \alpha=0.3)$. Figure 3.19 shows the plant output $y(t)$ tracking the sine-wave reference input $y_{r}$. Figure 3.20 shows the Bode plots of the true and identified plant respectively. 


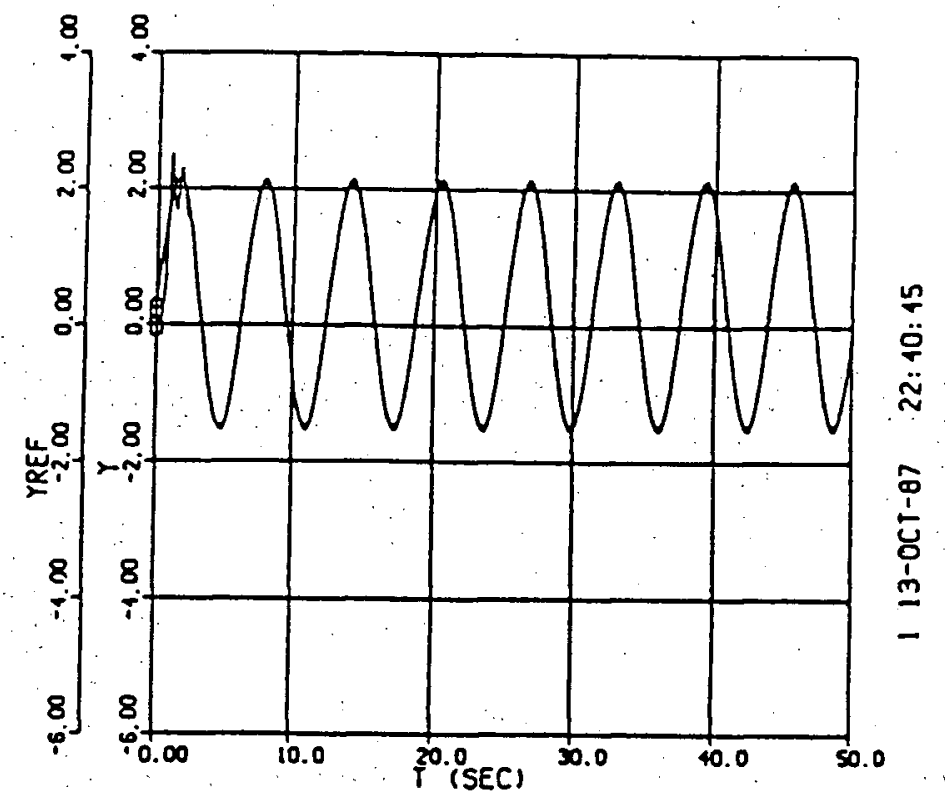

Figure 3.19: Example 3.6: Output tracking the reference input when $N=1, T=0.1, d=2$.

The identified Laguerre gain came out to be 1.45. If we evaluate the eigenvalues of the $A$-matrix from equation (3.123) we find out that for the above sampling frequency the system is stable. Increasing the sampling frequency to $T=0.01$, while keeping everything else the same, the system becomes eventually unstable.

Let us investigate more the instability mechanism when the sampling time is $T=$ $0.01 \mathrm{sec}$. According to Theorem 3.4 if the output model mismatch is bounded (i.e. equation (3.124) is true), then there is always a prediction horizon $d$ such that for every sampling time $T$ the closed loop self-tuning system remains stable. In this case if we increase $d$ we should expect system stability. Actually when the reference input is $1 \mathrm{rad} / \mathrm{s}$ we do get stability by increasing $d$. For values $d \geq 8$ the closed-loop system appears to be stable. Figure 3.21 shows the system output tracking the reference input when $d=10$.

Actually in the single parameter case $(N=1)$ the Laguerre state-space model 

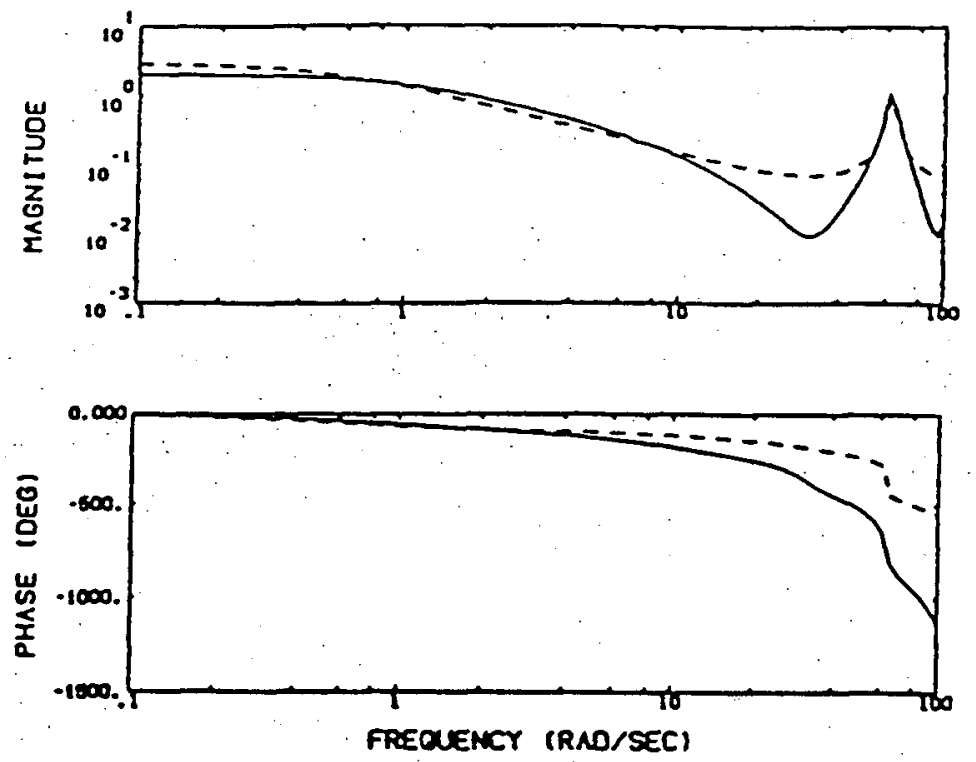

Figure 3.20: Example 3.6: Bode plots for the true (solid lines) and identified (dash lines) systems, when $N=1, T=0.1, d=2$.

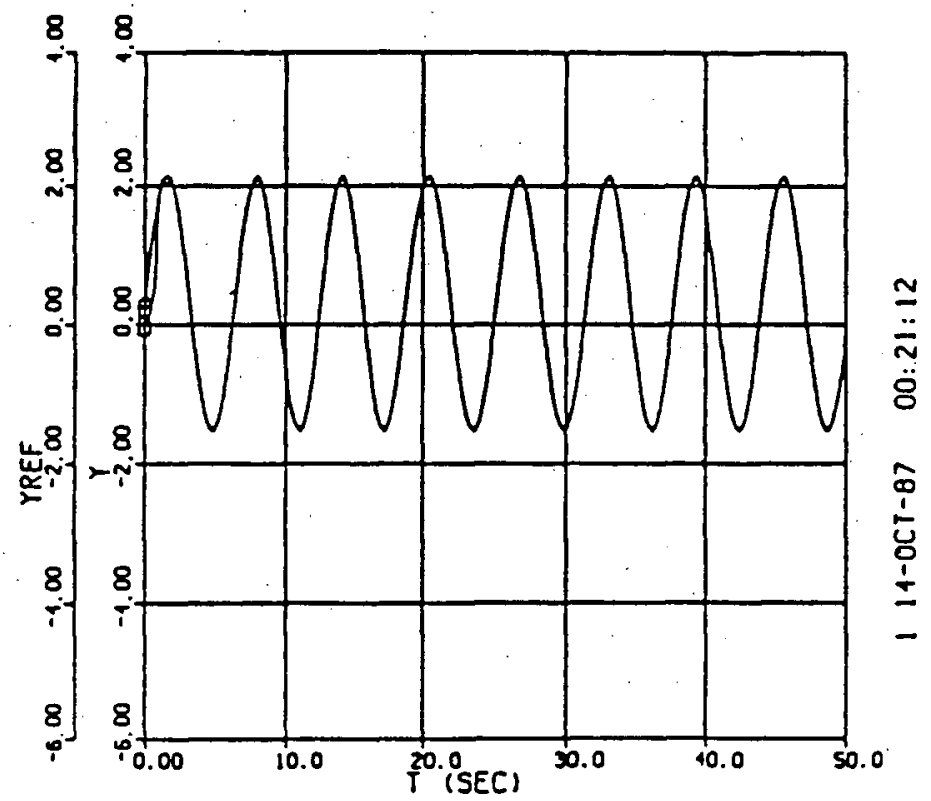

Figure 3.21: Example 3.6: System output tracking the reference input when $N=1, T=0.01, d=10$. (The 2 curves are almost identical) 
given by the set of equations $(3.117),(3.118)$ transforms to simple scalar expressions. The eigenvalues of the $\mathbf{A}_{\mathbf{c l}}$ matrix then can be evaluated from,

$$
\lambda_{i}\left(\mathbf{A}_{\mathrm{cl}}\right)=\lambda_{i}\left(A_{0}\right) \cup \lambda_{i}\left(A-\underline{b}\left(\left(1-\alpha^{d}\right) \underline{\hat{c}}^{T}+\underline{k}^{T}\right) \hat{\beta}^{-1}\right)
$$

Since the plant under study was assumed to be stable then the eigenvalues of the first term in the r.h.s. of equation (3.129) are always inside the unit disk. The second term in the r.h.s. of the above equation is a scalar expression and its value is always less than one as shown below (note that the terms, $A=\exp (-p T)>0$ and $b, \hat{c}, \hat{k}$ are now all scalars $\neq 0$, and $|\alpha|<1$ ),

$$
\begin{aligned}
& \left|A-b\left(\left(1-\alpha^{d}\right) \hat{c}+\hat{k}\right) \hat{\beta}^{-1}\right|=\left|A-\frac{b\left(\left(1-\alpha^{d}\right) \hat{c}+\hat{c}\left(A^{d}-1\right)\right)}{\left(\hat{c}\left(A^{d-1}+\ldots+1\right) b\right)}\right| \\
& =\left|A-\frac{A^{d}-\alpha^{d}}{\left(A^{d-1}+\ldots+1\right)}\right|=\left|\frac{A^{d-1}+\ldots+A+\alpha^{d}}{A^{d-1}+\ldots+A+1}\right|<1
\end{aligned}
$$

Thus all the eigenvalues of the $\mathbf{A}_{\mathrm{cl}}$ matrix are inside the unit circle and the closed-loop system is stable.

When the reference input $\omega$ was $1 \mathrm{rad} / \mathrm{s}$ stability was achieved by increasing $d$, because the output model mismatch was bounded. But the system appears to be unstable for any value of $d$ if the reference input is increased to $\omega=16.1 \mathrm{rad} / \mathrm{s}$. When condition (3.124) is not true then the stability of the closed loop system depends on the stability of the $A$-matrix in equation (3.123). It can be proved that for $T=0.01$ sampling time and for the particular plant of equation (3.128) the estimated Laguerre gain goes to zero (Dumont, 1988) and then the $A$-matrix in equation (3.123) has 2 eigenvalues outside of the unit disk, i.e the closed-loop system is unstable. The reason why the closed loop is unstable is that, for fixed $N=1$ the order of identification is inadequate either to represent sufficiently the dynamics of the given plant or make the model mismatch bounded at the specified sampling frequency and at the specified reference input. 

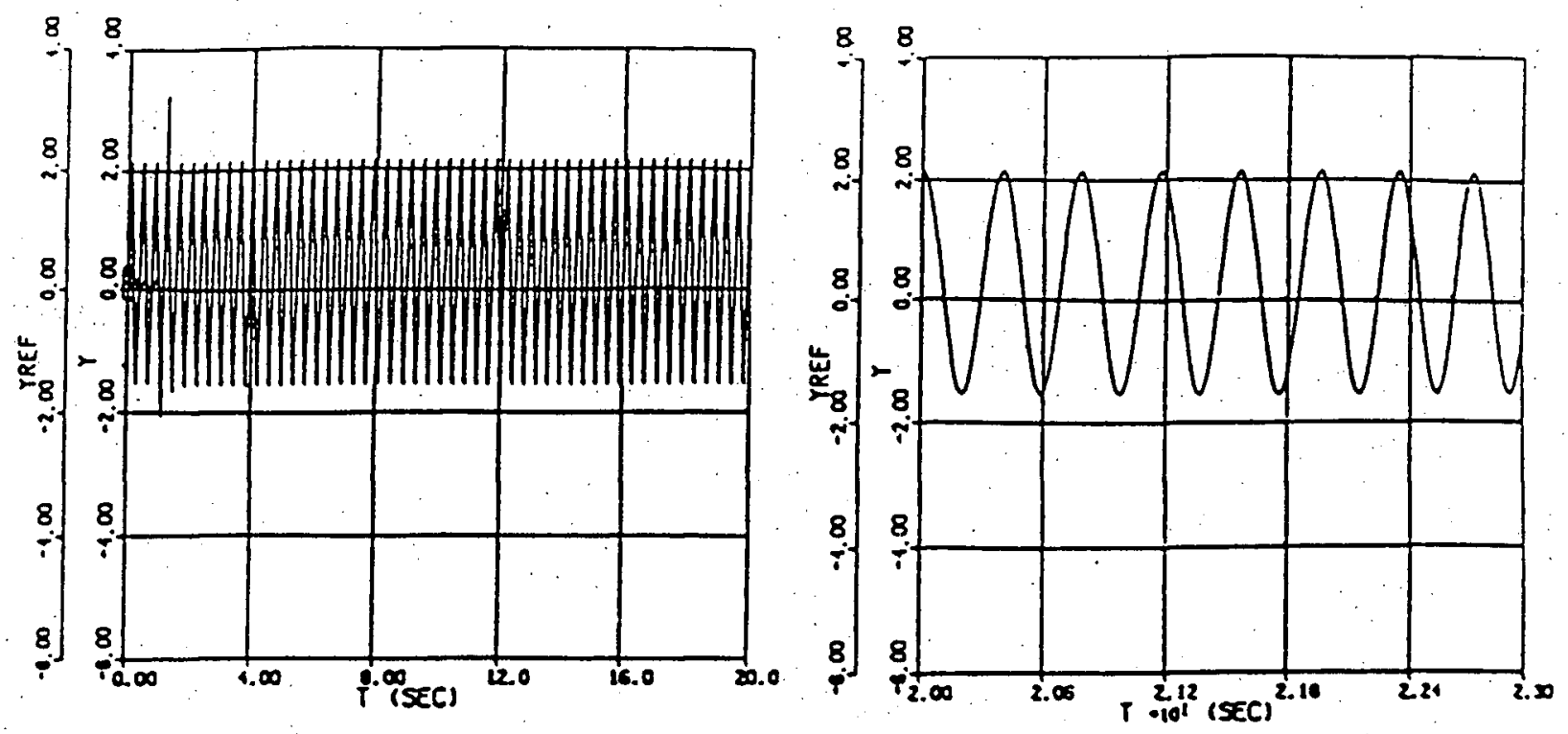

Figure 3.22: Example 3.6: (a) System output tracking the reference input when $N=2, T=0.01, d=2$. (b) An enlargment portion from $T=20$ to 23 . (The 2 curves are almost identical.)

In this case this leads to 2 possible solutions, either keep the identification error always bounded in case an a priori knowledge of the plant is available, or increase the order of the identification. By increasing the order of the estimation from 1 to 2 the system is always stable for values of sampling times 0.1 and 0.01 and for sinusoid reference inputs from 1 to $20 \mathrm{rad} / \mathrm{s}$. Figure 3.22 shows the output tracking the reference input and Figure 3.23 shows the bode plots of the true and the identified plant respectively $(N=2, T=0.01, p=0.5, d=2, \omega=16.1)$. By increasing the order of the identified model, stability has been achieved. Because the low-order Laguerre gains stay practically constant during an order increase, this can be done on-line without the system going through a transient phase. Flexibility in varying the dimensions of the problem on-line without system-upset is an advantage of this new controller. 

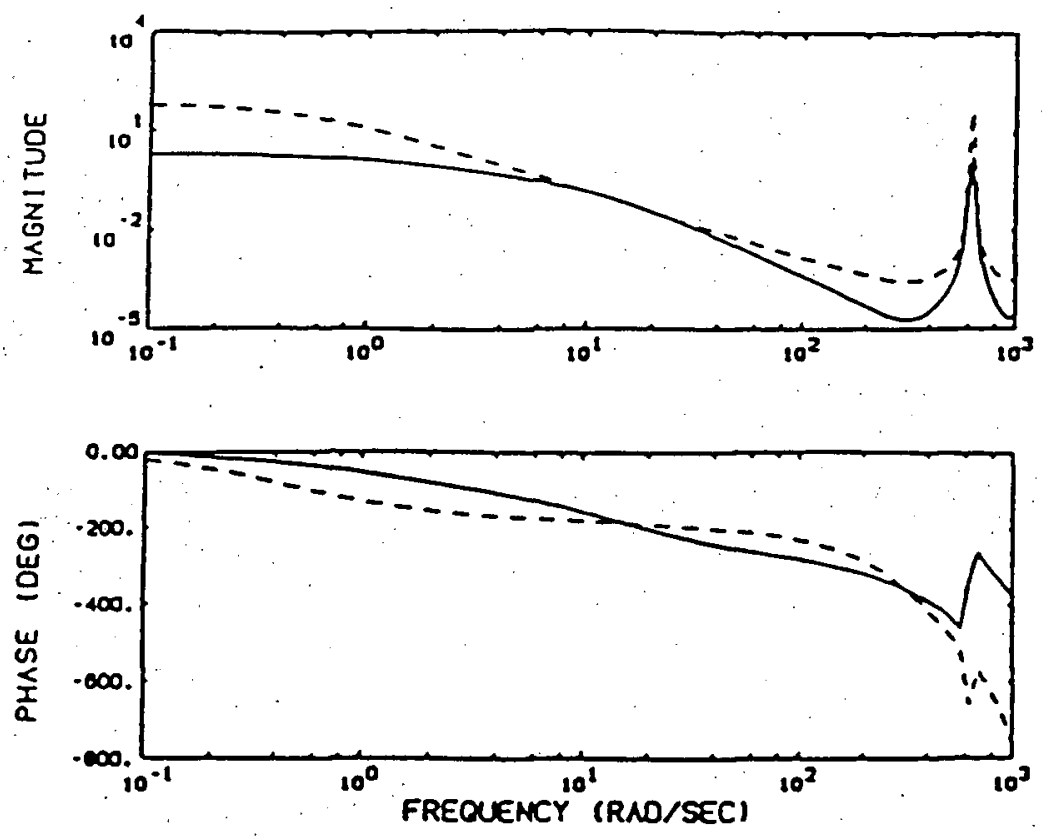

Figure 3.23: Example 3.6: Bode plots for true (solid lines) and identified (dash lines) systems, when $N=2, T=0.01, d=2$.

Example 3.7: Let a plant be described by the input-output equation:

$$
y(k)=0.9979 y(k-1)-0.0777 u(k-2)+e(k)-0.618 e(k-1)-0.378 e(k-2)
$$

At the $800^{\text {th }}$ sampling interval, we suddenly switch to the following plant,

$$
y(k)=0.934 y(k-1)-0.1102 u(k-9)+e(k)-0.559 e(k-1)-0.350 e(k-2)
$$

where $\mathrm{e}(\mathrm{t})$ is a white noise sequence $N(0,0.1)$. Figure 3.24 shows the output of the plant tracking a square wave set-point using the self-tuning scheme mentioned in Example 3.3. The parameters used were, $N=16, p=0.8, d=10$. Despite the sudden transition from one transfer function to another and despite the presence of noise the output is capable of following successfully the setpoint. 

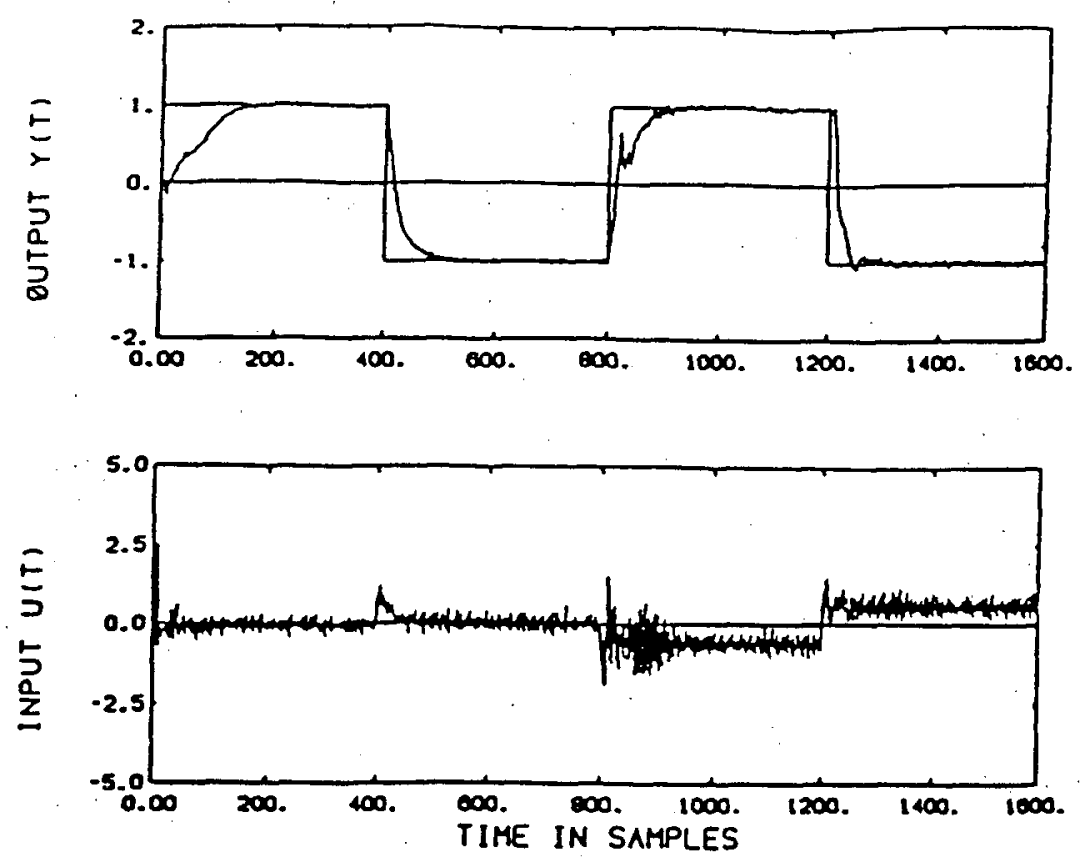

Figure 3.24: Example 3.7: System output tracking the reference input (above) and controller output (below). $\left(N=16, p=0.8, d=10, T=1.0\right.$. At the $800^{\text {th }}$ point we switch to a different plant):

\subsection{Practical Aspects and Implementation}

\subsubsection{Choice of Laguerre filter time constant}

Although, as found from simulations, the choice of the parameter $p$ used in the Laguerre ladder network is not crucial, it does influence the accuracy of the approximation of a given plant as a truncated Laguerre series. In the simulations, an extensive range of values for the parameter $p$ was found to give acceptable adaptation performance results for a given plant. A method to optimize the parameter $p$ that was tried in practice and found to perform very well was to store an array of plant input-output data for a period of time and then try a modified constrained Hooke-Jeeves optimization technique (Hooke and Jeeves, 1961) on them to obtain an optimum value for the parameter $p$ by minimizing the residual error obtained from the least squares identification. The method performed very well in practice with the only drawback being the additional 
memory requirement and the computational expense involved in allowing the direct search optimization routine to converge. Since at this time no analytical method is available for the choice of the parameter $p$, its choice is empirical, as explained below.

The ability to control the bandwidth of adaptive control schemes in order to avoid exciting unmodelled high frequencies dynamics is desirable for robustness. An interesting feature of the Laguerre ladder network is that the first block is a first-order low-pass filter with cut-off frequency $1 / p$ and the rest of the blocks are all-pass filters. This provides some filtering qualities to the Laguerre self-tuner and allows some control over its bandwidth. Thus the choice of the parameter $p$ can be made as to have $1 / p$ roughly around the cross-over angular velocity $\omega$ of the plant.

Moreover if a plant with a long time delay $k_{d}$ is to be controlled then because of an apparent similarity of the Laguerre functions to Padé approximants a suitable choice for $p$ can be made. The Padé approximation (in Laplace transform) of a continuous time delay $k_{d}$ is given by,

$$
k_{d}=\lim _{n \rightarrow \infty}\left(\frac{1-\frac{k_{d} \delta}{2 n}}{1+\frac{k_{d A}}{2 n}}\right)^{n}
$$

The above representation resembles the all-pass phase-shift block chain encountered in the Laguerre Ladder Network (figure 2.2). A good choice for $p$ would then be, $p=2 n / k_{d}$.

\subsubsection{Number of filters}

The number of filters required in the representation relates primarily to the presence of underdamped modes and the time delay in the plant. The reason is that the orthonormal filters are used to model all dynamics including the delay. For low-order plants with significant delay relative to the dominant time constant, simulations show that 5-10 filters give satisfactory results in many cases. For high-order underdamped plants 
with substantial delays the number has to increase from 10-15 filters. Of course, when the delay is not substantial, fewer filters are required. By monitoring the Laguerre spectrum or the residual error on-line, it is easy to vary the dimension of the problem by increasing or decreasing the order of the identification.

\subsubsection{Choice of $d$ and $\alpha$}

The driver block pole relates to the desired performance and is easy to choose. The prediction horizon can be automatically altered to make sure that $\hat{\beta}$ is nonzero and that non-minimum phase zeros are not cancelled. The latter can be achieved by checking the roots of equation (3.81). A simpler method is to choose $d$ such that $\hat{\beta}$ is of the same sign as the estimated process gain, and of significant amplitude. A simple criterion can be

$$
\hat{\beta} \geq \epsilon \underline{\hat{c}}^{T}(I-A)^{-1} \underline{b}
$$

where $\epsilon \approx 0.5$ and $(I-A)^{-1} \underline{b}$ can be precomputed, as it does not depend on the estimates. 


\section{Chapter 4}

\section{Stochastic Adaptive Control}

\subsection{Introduction}

The development presented in Chapter 3 concerning the formulation and test of a deterministic self-tuning scheme based on Laguerre functions will now be used to build an explicit stochastic self-tuning scheme. All real processes are corrupted with external disturbances and there is always a measurement noise involved when measuring output signals. Thus it would be good to extend the ideas presented in the previous chapter and construct a self-tuning scheme suitable for a stochastic environment. A stochastic selftuning controller is presented here that makes use of easily understood concepts showing simplicity and flexibility while capable of achieving good and robust control. Although Laguerre functions were chosen, other orthonormal functions may be used. Some other potential sets with success were tested, as it will be shown in a subsequent chapter. The scheme presented here retains all the nice properties mentioned for the deterministic case in Chapter 3. In addition, the identified model of the external disturbances is taken in account in the implementation of the control law. Simulation results are also presented to support and demonstrate the excellent behaviour, capabilities and ease of use of the proposed algorithm. 


\subsection{An explicit stochastic control law}

Models of the physical plant and of the stochastic noise environment are constructed respectively based on the representation outlined in the previous section 2.3 . This is done by modelling the deterministic part (see Figure 4.25 ) with time-scale $p$, as:

$$
\begin{gathered}
\underline{l}(t+1)=A \underline{l}(t)+\underline{b} u(t) \\
y_{l}(t)=\underline{c}^{T} \underline{l}(t)
\end{gathered}
$$

and the stochastic part, with time scale $p^{\prime}$, by a second network:

$$
\begin{gathered}
\underline{n}(t+1)=A^{\prime} \underline{n}(t)+\underline{b}^{\prime} e(t) \\
y_{n}(t)=\underline{c}^{\prime} \underline{n}(t)+e(t)
\end{gathered}
$$

Where $\underline{l}(t)$ and $\underline{n}(t)$ are the $N$ and $N^{\prime}$-dimensional plant and noise state-vectors respectively, and $e(t)=N(0,1)$ white noise. The parameter vectors are defined as, $\underline{c}^{T}=\left[\begin{array}{lll}c_{1} & c_{2} & \ldots \\ c_{N}\end{array}\right]$ and, $\underline{c}^{\prime T}=\left[\begin{array}{lll}c_{1}^{\prime} & c_{2}^{\prime} \ldots c_{N^{\prime}}^{\prime}\end{array}\right]$, respectively.

Combining equations $(4.135,4.137)$ the plant process output can be represented as,

$$
y(t)=y_{l}(t)+y_{n}(t)=\underline{c}^{T} \underline{l}(t)+\underline{c}^{T} \underline{n}(t)+e(t)
$$

Note that, $A, \underline{b}, A^{\prime}, \underline{b}^{\prime}$, above are a-priori known while only the $\underline{c}$, $\underline{c}^{\prime}$ have to be estimated online, e.g: by using a Recursive Extended Least Squares (RELS) identification method (Ljung and Soderstrom, 1983).

A predictive exression will now be derived for the plant output based on the above orthonormal representation. From equation (4.138) while using equations (4.135), (4.137) we can write a $d$-steps ahead predictive formula for $y(t)$ as,

$$
y(t+d)=\underline{c}^{T} \underline{l}(t+d)+\underline{c}^{T} \underline{n}(t+d)+e(t+d)
$$




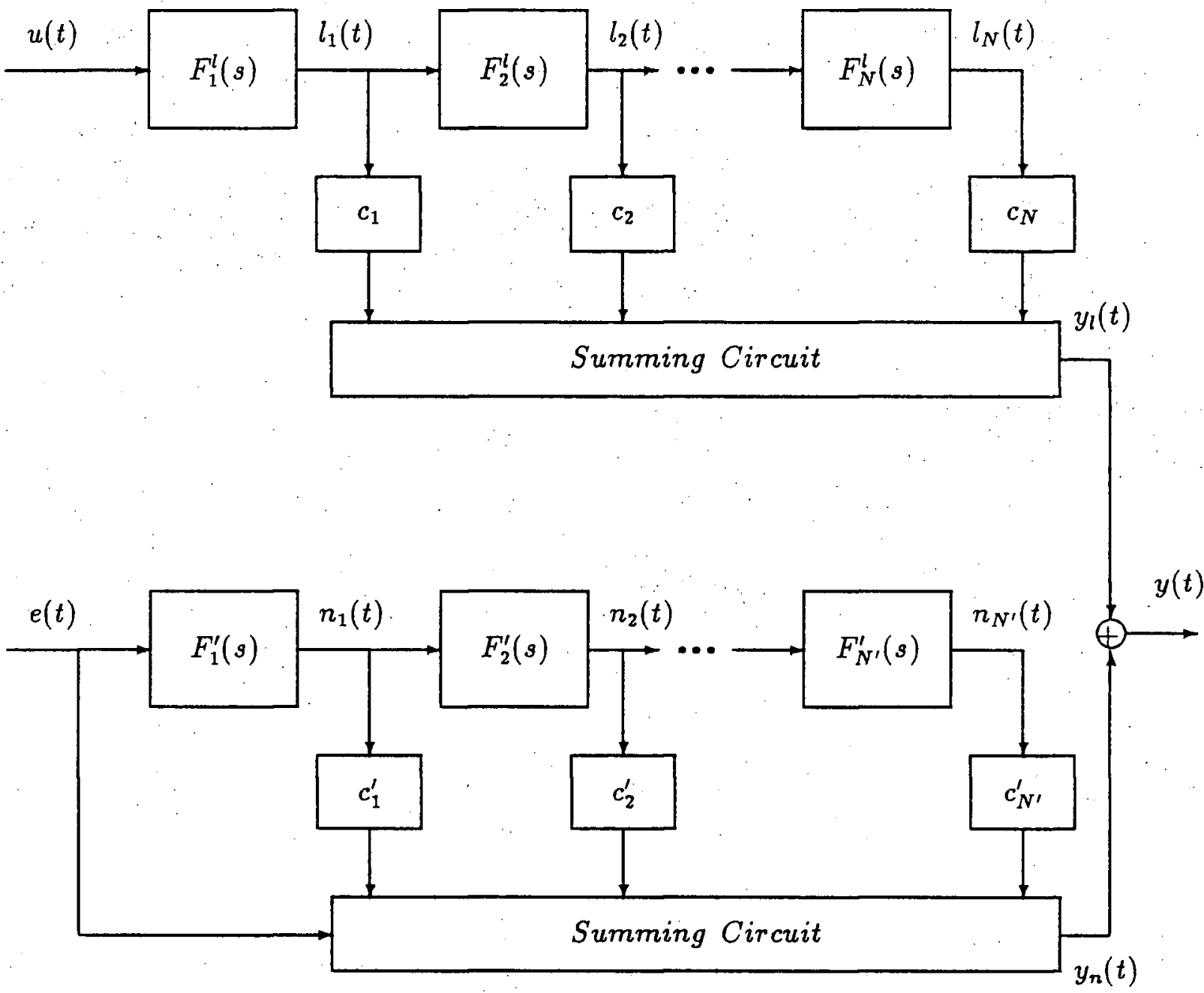

Figure 4.25: Orthonormal Modelling of a stochastic system 


$$
\begin{aligned}
& +y(t)-\underline{c}^{T} \underline{l}(t)-\underline{c}^{T} \underline{n}(t)-e(t) \\
= & \underline{c}^{T}[\underline{l}(t+d)-\underline{l}(t)]+\underline{c}^{T}[\underline{n}(t+d)-\underline{n}(t)] \\
& +e(t+d)-e(t)+y(t)
\end{aligned}
$$

Now if we derive predictive expressions, based on information up to time $t$, for $\underline{l}(t+d)$ and $\underline{n}(t+d)$ using the same recursive method described in Section 3.1 for $\underline{l}(t+d)$, while assuming $u(t)=u(t+1)=\cdots=u(t+d-1)$, equation (4.139) gives,

$$
\begin{aligned}
y(t+d)= & \underline{c}^{T}\left[\left(A^{d}-I\right) \underline{l}(t)+\left(A^{d-1}+\cdots+I\right) \underline{b} u(t)\right] \\
& +\underline{c}^{\prime T}\left[\left(A^{\prime d}-I\right) \underline{n}(t)+A^{\prime d-1} \underline{b}^{\prime} e(t)+A^{\prime d-2} \underline{b}^{\prime} e(t+1)+\cdots+\underline{b}^{\prime} e(t+d-1)\right] \\
& +e(t+d)-e(t)+y(t)
\end{aligned}
$$

By ignoring future noise terms and using the Certainty Equivalence principle the best $d$-steps ahead $\left(d>k_{d}\right)$ predictive expression for the system output $y(t)$ can be derived as;

$$
\begin{aligned}
\hat{y}(t+d \mid t)= & \underline{c}^{T}\left[\left(A^{d}-I\right) \underline{l}(t)+\left(A^{d-1}+\cdots+I\right) \underline{b} u(t)\right] \\
& +\underline{c}^{\prime T}\left(A^{\prime d}-I\right) \underline{n}(t)+\left(\underline{c}^{\prime T} A^{\prime d-1} \underline{b}^{\prime}-1\right) \hat{e}(t)+y(t)
\end{aligned}
$$

If we define a prescribed reference trajectory $y_{\tau}(t+d)$ (as we did in Section 3.1) and equate it to the right-hand side of equation (4.141), we can solve for the required control input signal $u(t)$,

$$
u(t)=\left[y_{r}(t+d)-y(t)-\underline{P}^{T} \underline{l}(t)-\underline{P}^{T} \underline{n}(t)-\gamma \hat{e}(t)\right] / \beta
$$

where,

$$
\begin{gathered}
\beta=\underline{c}^{T}\left(A^{d-1}+\ldots+I\right) \underline{b} \\
\gamma=\underline{c}^{r T} A^{\prime d-1} \underline{b}^{\prime}-1 \\
\underline{P}^{T}=\underline{c}^{T}\left(A^{d}-I\right)
\end{gathered}
$$




$$
\underline{P}^{\prime T}=\underline{c}^{r T}\left(A^{\prime d}-I\right)
$$

The output prediction error can be computed from equations $(4.140),(4.141)$ as,

$$
\begin{aligned}
\tilde{y}(t+d) & =y(t+d)-\hat{y}(t+d) \\
& =\underline{c}^{\prime T}\left[\dot{A}^{\prime d-2} \underline{b}^{\prime} e(t+1)+\cdots+\underline{b}^{\prime} e(t+d-1)\right]+e(t+d)
\end{aligned}
$$

Equation (4.143) reveals that the prediction error is a Moving Average noise sequence of order $(d-1)$.

The new stochastic self-tuning algorithm makes use of equation (4.138) for recursively identifying the parameter vectors $\underline{c}$ and $\underline{c}^{\prime}$ using an extended least-squares (RELS) identification, and equation (4.142) for the calculation of the required control input. The RELS algorithm follows the standard algorithm that has been reported before in the literature, (Ljung and Soderstrom, 1983). The control law requires also an estimate of the noise $\hat{e}(t)$ which can be computed as a residual from the identification. To include immunity against bias terms during the identification a solution similar to the one presented in Clarke, Hodgson and Tuffs (1983) was adopted to solve the offset problem. An advantage of this technique is that an estimate of the prediction error can be computed at the same time. In particular, for the evaluation of the required noise estimate we can write,

$$
y(t)=\underline{s}^{T} \underline{l}(t)+\underline{c}^{T} \underline{n}(t)+\hat{e}(t)
$$

where $\hat{e}(t)$ is an estimate of the prediction error $\tilde{y}(t \mid t-d)$. The above equation (4.144) can also be written as,

$$
y(t-d)=\underline{c}^{T} \underline{l}(t-d)+\underline{c}^{T} \underline{n}(t-d)+\hat{e}(t-d)
$$

By subtracting equation (4.145) from (4.144) we get,

$$
y(t)=y(t-d)-\hat{e}(t-d)+\underline{c}^{T} \Delta_{d} l(t)+\underline{c}^{T} \Delta_{d} \underline{n}(t)+\hat{e}(t)
$$


where $\Delta_{d}$ stands for the $d$-order difference operator $\left(1-q^{-d}\right)$. In the unknown parameter case the prediction error will not be available so its value must be proxied. This is usually done by computing the residuals of a previous estimation i.e. solving equation (4.146) while using the parameter estimates from the previous step. For the RELS algorithm we define the new observation $z(t)$ to be,

$$
z(t)=y(t)-y(t-d)+\hat{e}(t-d)
$$

and the following equation is employed for the RELS,

$$
z(t)=\underline{c}^{T} \Delta_{d} l(t)+\underline{c}^{T} \Delta_{d} \underline{n}(t)+e^{\prime}(t)
$$

where $e^{\prime}(t)$ is computed from the residuals of the identification. For the required noise estimate we use,

$$
\hat{e}(t)=e^{\prime}(t)-\bar{e}(t)
$$

where a filtered proxying of $e(t)$ has been employed by using the equation,

$$
\bar{e}(t)=\tau \bar{e}(t-1)+(1-\tau) e^{\prime}(t)
$$

The choice of the constant $\tau$ above in the ligh pass filter is by no means crucial and good results can be obtained for values in the range $(0,0.999]$. The value $\hat{e}(t)$ derived above is then used as the required noise estimate in the computation of the control law.

\subsubsection{Stability analysis}

The Laguerre orthonormal network (Figure 4.25) which was used as the basic model of a stochastic dynamic system was expressed in state-space form by equations (4.135) and (4.137). Note that the control law given by equation (4.142) is also in state-feedback form. Actually the whole closed loop system can be put in a state-space form. 
Assume that the true stochastic dynamic system is represented by the following state-space equations where its deterministic part is $\mathbf{L}_{2}[0, \infty)$ :

$$
\begin{gathered}
\underline{x}(t+1)=A_{0} \underline{x}(t)+\underline{b}_{0} u(t)+\Gamma w(t) \\
y(t)=\underline{c}_{0}^{T} \underline{x}(t)+v(t)
\end{gathered}
$$

where $w(t)$ and $v(t)$ are bounded stochastic signals and have finite covariances. And let the stochastic Laguerre ladder network model of the above plant be represented by the pair of state-space descriptions, as in Section 4.2, i.e. the deterministic part as,

$$
\begin{gathered}
\underline{l}(t+1)=A \underline{l}(t)+\underline{b} u(t) \\
\hat{y}_{l}(t)=\underline{\hat{c}}^{T} \underline{l}(t)
\end{gathered}
$$

and the stochastic part as,

$$
\begin{gathered}
\underline{n}(t+1)=A^{\prime} \underline{n}(t)+\underline{b}^{\prime} e(t) \\
\hat{y}_{n}(t)=\underline{\underline{c}}^{\prime} \underline{n}(t)+e(t)
\end{gathered}
$$

where the state vectors, $\underline{x}, \underline{l}, \underline{n}$, are not necessarily of the same order.

Assume that, the system is under self-tuning control using the stochastic predictive control approach described earlier in Section 4.2. For simplicity, let a recursive extended least squares (RELS) identification technique be employed for the estimation of the parameter vectors, $\underline{c}$ and $\underline{c}^{\prime}$. Using the certainty equivalence principle the estimates, $\hat{\underline{c}}$ and $\underline{\hat{c}}^{\prime}$, are taken as the true ones and substituted back in the controller equation. Then all the equations that follow hold for any given discrete time $t$ during the operation of the closed-loop self-tuning system.

The controller output is given by equation (4.142),

$$
u(t)=\left[y_{r}(t+d)-y(t)-\underline{P}^{T} \underline{l}(t)-\underline{P}^{\prime T} \underline{n}(t)-\gamma \hat{e}(t)\right] / \beta
$$


where,

$$
\begin{gathered}
\beta=\underline{c}^{T}\left(A^{d-1}+\ldots+I\right) \underline{b} \\
\gamma=\underline{c}^{T} A^{\prime d-1} \underline{b}^{\prime}-1 \\
\underline{P}^{T}=\underline{c}^{T}\left(A^{d}-I\right) \\
\underline{P}^{T}=\underline{c}^{T}\left(A^{\prime d}-I\right)
\end{gathered}
$$

Assume the constant $\beta$ to be bounded from below. Substituting equation (4.157) in equations (4.151) and (4.153) we get,

$$
\begin{aligned}
\underline{x}(t+1)= & \left(A_{0}-\underline{b}_{0} \underline{c}_{0}^{T} / \beta\right) \underline{x}(t)-\underline{b}_{0} \underline{P}^{T} \underline{l}(t) / \beta-\underline{b}_{0} \underline{P}^{\prime T} \underline{n}(t) / \beta-\underline{b}_{0} \gamma \hat{e}(t) / \beta \\
& -\underline{b}_{0} v(t) / \beta+\Gamma w(t)+\underline{b}_{0} y_{r} / \beta \\
\underline{l}(t+1)= & -\underline{b}_{0}^{T} \underline{x}(t) / \beta+\left(A-\underline{b} \underline{P}^{T} / \beta\right) \underline{l}(t)-\underline{b} \underline{P}^{\prime T} \underline{n}(t) / \beta-\underline{b} \gamma \hat{e}(t) / \beta \\
& -\underline{b} v(t) / \beta+\underline{b} y_{r} / \beta
\end{aligned}
$$

And for the noise network we can write from equation (4.155),

$$
\underline{n}(t+1)=A^{\prime} \underline{n}(t)+\underline{b}^{\prime} \hat{e}(t)
$$

where $\hat{e}(t)$ using equations $(4.149),(4.150)$, can be written as,

$$
\begin{aligned}
\hat{e}(t)= & \tau(y(t)-y(t-d))+\tau \hat{e}(t-d)-\tau \underline{c}^{T}(\underline{l}(t)-\underline{l}(t-d)) \\
& -\tau \underline{c}^{T}(\underline{n}(t)-\underline{n}(t-d))-\tau \bar{e}(t-1)
\end{aligned}
$$

Introducing a new state variable $\tilde{q}(t)$,

$$
\tilde{q}(t) \equiv \hat{e}(t)-\tau \underline{c}_{0}^{T} \underline{x}(t)+\tau \underline{c}^{T} \underline{l}(t)+\tau \underline{c}^{T} \underline{\underline{n}}(t)
$$

we can rewrite for the noise estimate,

$$
\hat{e}(t)=\tilde{q}(t)+\tau \underline{c}_{0}^{T} \underline{x}(t)-\tau \underline{c}^{T} \underline{l}(t)-\tau \underline{c}^{T} \underline{n}(t)
$$


Substituting equation (4.163) into equations $(4.158),(4.159),(4.160)$, we get,

$$
\begin{aligned}
\underline{x}(t+1)= & \left(A_{0}-\underline{b}_{0} \underline{c}_{0}^{T} / \beta-\underline{b}_{0} \gamma \tau \underline{c}_{0}^{T} / \beta\right) \underline{x}(t)+\left(-\underline{b}_{0} \underline{P}^{T} / \beta+\underline{b}_{0} \gamma \tau \underline{c}^{T} / \beta\right) \underline{l}(t) \\
& +\left(-\underline{b}_{0} \underline{P}^{T} / \beta++\underline{b}_{0} \gamma \tau \underline{c}^{T} / \beta\right) \underline{n}(t)-\underline{b}_{0} \gamma \tilde{q}(t) / \beta \\
& -\underline{b}_{0} v(t) / \beta+\Gamma w(t)+\underline{b}_{0} y_{\tau} / \beta \\
\underline{l}(t+1)= & \left(-\underline{b}_{0} \underline{c}_{0}^{T} / \beta-\underline{b} \gamma \tau \underline{c}_{0}^{T} / \beta\right) \underline{x}(t)+\left(A-\underline{b} \underline{P}^{T} / \beta+\underline{b} \gamma \tau \underline{c}^{T} / \beta\right) \underline{l}(t) \\
& +\left(-\underline{b} \underline{P}^{T} / \beta+\underline{b} \gamma \tau \underline{c}^{T} / \beta\right) \underline{n}(t)-\underline{b} \gamma \tilde{q}(t) / \beta \\
& -\underline{b} v(t) / \beta+\underline{b} y_{r} / \beta
\end{aligned}
$$

And for the noise state-vector we get,

$$
\begin{aligned}
\underline{n}(t+1)= & \underline{b}^{\prime} \tau \underline{c}_{0}^{T} \underline{x}(t)-\underline{b}^{\prime} \tau \underline{c}^{T} \underline{l}(t) \\
& +\left(A^{\prime}-\underline{b}^{\prime} \tau \underline{c}^{T}\right) \underline{n}(t)+\underline{b}^{\prime} \tilde{q}(t)
\end{aligned}
$$

We can also write the following expression for the noise sequence using equation (4.163),

$$
\begin{aligned}
\bar{e}(t)= & \bar{e}(t-1)+(1-\tau) \hat{e}(t) / \tau \\
= & \bar{e}(t-1)+(1-\tau) \tilde{q}(t) / \tau+(1-\tau) \underline{c}_{0}^{T} \underline{x}(t) \\
& -(1-\tau) \underline{c}^{T} \underline{l}(t)-(1-\tau) \underline{c}^{T} \underline{n}(t)
\end{aligned}
$$

Introducing a new state variable $r(t)$ and using equation (4.167) we can write,

$$
\begin{aligned}
r(t) \equiv & \bar{e}(t)-(1-\tau) \tilde{q}(t) / \tau-(1-\tau) \underline{c}_{0}^{T} \underline{x}(t) \\
& +(1-\tau) \underline{c}^{T} \underline{l}(t)+(1-\tau) \underline{s}^{\tau} \underline{n}(t) \\
= & \bar{e}(t-1)
\end{aligned}
$$

Solving equation (4.168) w.r.t. $\bar{e}(t)$ and back substituting we can get,

$$
\begin{aligned}
r(t+1)= & r(t)+(1-\tau) \tilde{q}(t) / \tau+(1-\tau) \underline{\underline{c}}_{0}^{T} \underline{\underline{x}}(t) \\
& -(1-\tau) \underline{c}^{T} \underline{l}(t)-(1-\tau) \underline{c}^{\prime} \underline{n}(t)
\end{aligned}
$$


And finally equations (4.150),(4.162) and (4.169) give,

$$
\begin{aligned}
q(t+1)= & (\tau-1) \bar{q}(t)+(\tau-1) \tau \underline{c}_{0}^{T} \underline{x}(t)+(1-\tau) \tau \underline{c}^{T} \underline{l}(t) \\
& +(1-\tau) \tau \underline{\underline{c}}^{T} \underline{n}(t)-\tau r(t)+(\tau-1) \tau \underline{c}_{0}^{T} \underline{x}(t-d+1) \\
& +(1-\tau) \tau \underline{c}^{T} \underline{l}(t-d+1)+(1-\tau) \tau \underline{c}^{T} \underline{n}(t-d+1)+\tau q(t-d+1) \\
& -\tau v(t+1)+\tau v(t-d+1)
\end{aligned}
$$

Now if we ignore any future noise terms, equations (4.164), (4.165), (4.166), and equations (4.169), (4.170) represent the whole closed-loop system in state-space form. The state vector of this representation is defined as,

$$
\begin{aligned}
& \underline{\mathrm{X}}_{\mathrm{cl}}{ }^{\top}(t)= \\
& {\left[\begin{array}{llllllll}
\underline{x}(t) & \underline{l}(t) & \underline{n}(t) & \tilde{q}(t) & \underline{x}(t-1) & \underline{l}(t-1) & \underline{n}(t-1) & q(t-1)
\end{array}\right.}
\end{aligned}
$$

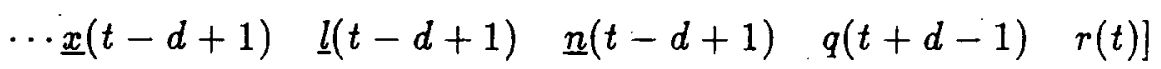

The A-matrix $\left(\mathbf{A}_{\mathbf{C L}}\right)$ of this closed-loop state-space representation can be studied for stability purposes. The closed-loop system is stable if all its eigenvalues are inside the unit disk. The order of this matrix is,

$$
\operatorname{deg}\left(\mathbf{A}_{\mathbf{C L}}\right)=(d-1) \times(\operatorname{deg}(\underline{x})+\operatorname{deg}(\underline{l})+\operatorname{deg}(\underline{n})+1)+1
$$

An implicit stochastic control scheme has also been derived and tested with success using the new Laguerre series representation. Its derivation follows along the same lines as described in Section 3.2.2.

\subsubsection{Practical Aspects and Simulation}

The previously described self-tuning approach has been succesfully implemented in plant simulations using efficient and fast code algorithms. For a thorough discussion 
of the choice of the Laguerre time-scale, $p$, the number of filters, the horizon $d$ and the reference trajectory $y_{r}(t)$, plus other discussions related to the practical aspects of the new self-tuner, the reader is referred to section 3.5.1 and to Dumont and Zervos (1986) or Zervos and Dumont (1988a). A modified Hooke-Jeeves (Hooke and Jeeves, 1961) search optimization method has been implemented to search for an optimal value of the positive real time-scale $p$. Plants whose impulse responses were not $\mathbf{L}_{2}[0, \infty)$ functions have also been tested in simulations and the self-tuner worked satisfactorily. The simulation results that follow show the good behaviour and flexibility of the stochastic scheme.

Example 4.1: This example demonstrates the behaviour and applicability of the newly proposed scheme. It also demonstrates a good example on the flexibility of the choice of the Laguerre parameters. Consider the following non-minimum phase plant,

$$
\begin{aligned}
y(t)= & 1.5 y(t-1)-0.7 y(t-2)+u\left(t-k_{d}\right) \\
& +1.5 u\left(t-k_{d}-1\right)+e(t)-0.7 e(t-1) \\
& +0.4 e(t-2)+0.25 e(t-3)+0.87 e(t-4)
\end{aligned}
$$

where, $k_{d}$ is the plant time-delay. Table 4.2 shows analytically the simulation runs with different choices of, the time-delay $k_{d}$, the number of filters $N$ and $N^{\prime}$, and the Laguerre time-scales $p, p^{\prime}$ respectively. The set-point was a square wave of amplitude 1 and the $1^{\text {ot }}$-order filter constant of the reference trajectory $y_{r}$ was set to $\alpha=0.7$ in all runs.

In all the simulations, the initial parameter estimates were zero, the initial covariance matrix $100 \times I$, the forgetting factor 1.0, a step load-disturbance of $30 \%$ of the step change was applied at $t=240$ and $t=280$, and the noise $e(t)$ was zero-mean white with $\sigma=0.1$. For every simulation run, 2000 sampling points are shown, recorded after the parameter identification has converged. Because of the irregular initial transient period, one can either, (a) perform an open-loop identification first and then apply the 


\begin{tabular}{|c||c|c|c|c|c|c|}
\hline \multicolumn{7}{|c|}{ Table 4.2 } \\
\hline \hline & $K_{d}$ & $d$ & $N$ & $p$ & $N^{\prime}$ & $p^{\prime}$ \\
\hline Fig. 4.26 & 1 & 2 & 4 & 0.3 & 4 & 0.1 \\
\hline Fig. 4.27 & 1 & 2 & 4 & 6.0 & 4 & 0.5 \\
\hline Fig. 4.28 & 1 & 2 & 8 & 0.1 & 3 & 0.2 \\
\hline Fig. 4.29 & 1 & 3 & 8 & 0.3 & 4 & 0.8 \\
\hline Fig. 4.30 & 1 & 6 & 8 & 0.4 & 2 & 0.5 \\
\hline Fig. 4.31 & 3 & 5 & 8 & 0.4 & 2 & 0.5 \\
\hline Fig. 4.32 & 3 & 5 & 8 & 4.0 & 2 & 0.5 \\
\hline Fig. 4.33 & 6 & 8 & 12 & 0.3 & 2 & 0.5 \\
\hline
\end{tabular}

Table 4.2: Example 4.1: The choice of the various parameters during the simulation runs.

control action by closing the loop, or (b) start with the loop closed but with some saturation limits on the input to limit the output deviations, or (c) apply a control input with a commissioning period. The graphs were produced using the method (b) above starting at time -400 and with input limits \pm 50 . The purpose of the experiment was to test the steady-state performance of the stochastic self-tuner using a different and mixed choice of parameters. (see Figures, 4.26, 4.27, 4.28, for system response comparisons). Figures, 4.29, 4.30 show the flexibility on the choice of the prediction horizon $d$. Figures, 4.31, 4.32 show two typical runs of a plant with a moderate time-delay when only the Laguerre time-scale $p$ of the deterministic ladder network has been changed by a magnitude of order 10. Figure 4.33 shows the performance for a plant with larger time delay. Figure 4.34 shows the output performance when the implicit scheme was applied to the same plant as in Case 8 in Table $4.2,\left(K_{d}=6, d=8, N=12, p=0.3\right)$. The results indicate that the self-tuner performed well even with different, time-scales, number of filters, and prediction horizons. It was also capable of handling relatively large delays (Figures : 4.33, 4.34). 


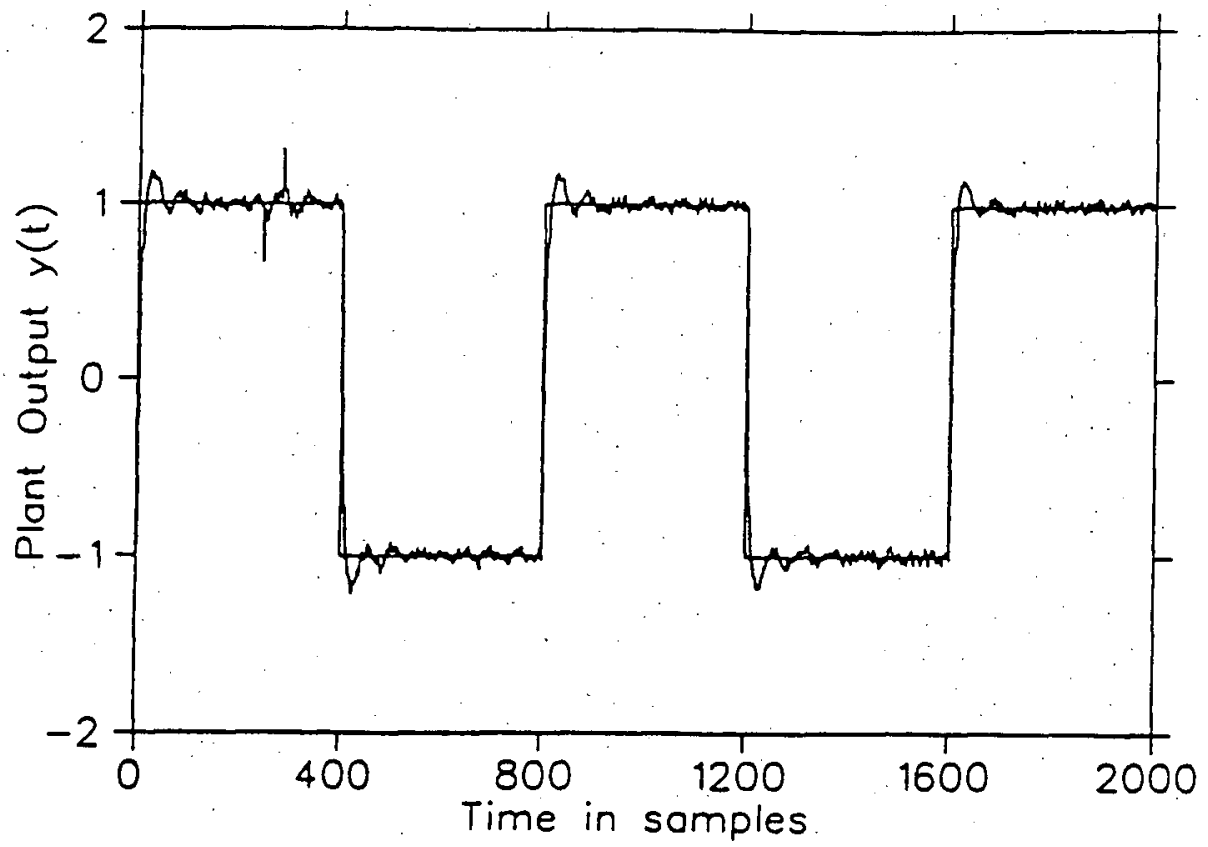

Figure 4.26: Example 4.1: Explicit stochastic self-tuner. System output tracking a square wave set-point. Case 1 of Table 4.2 .

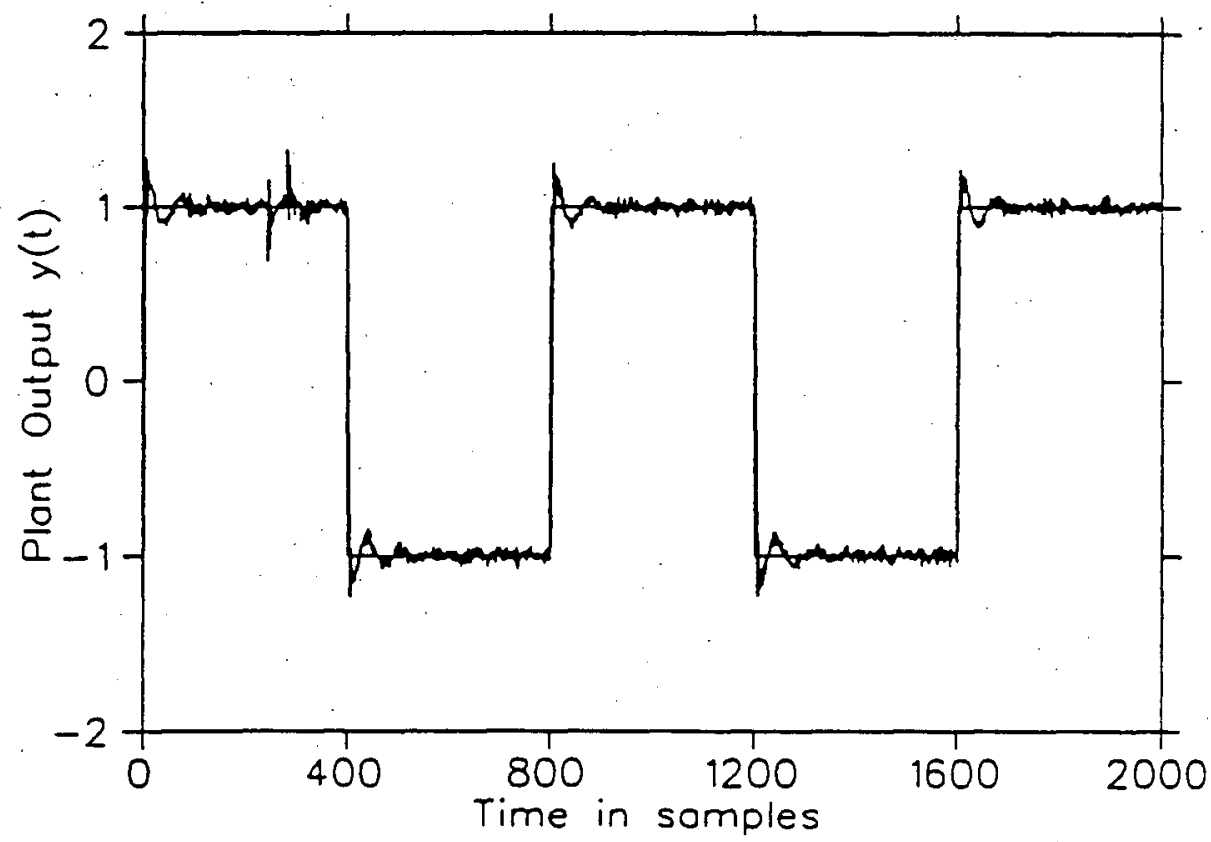

Figure 4.27: Example 4.1: Explicit stochastic self-tuner. System output tracking a square wave set-point. Case 2 of Table 4.2 . 


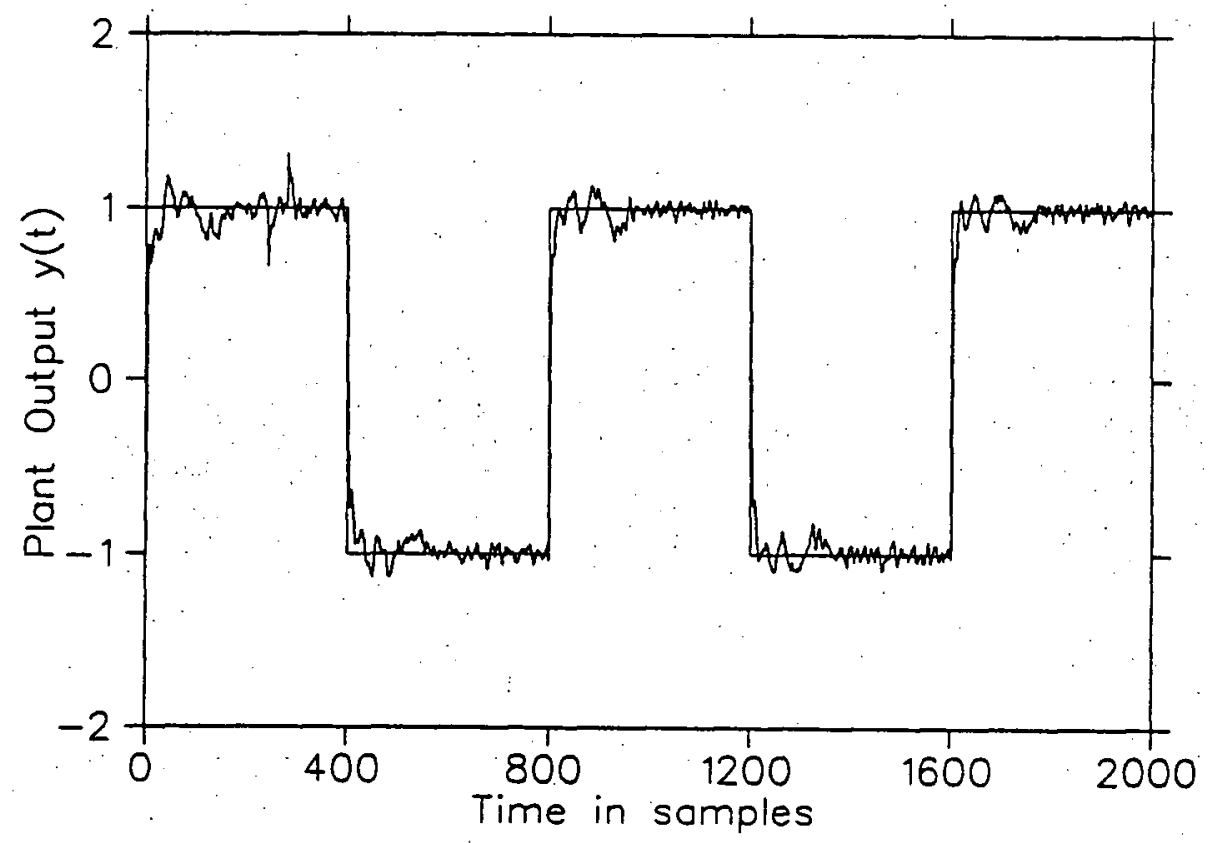

Figure 4.28: Example 4.1: Explicit stochastic self-tuner. System output tracking a square wave set-point. Case 3 of Table 4.2 .

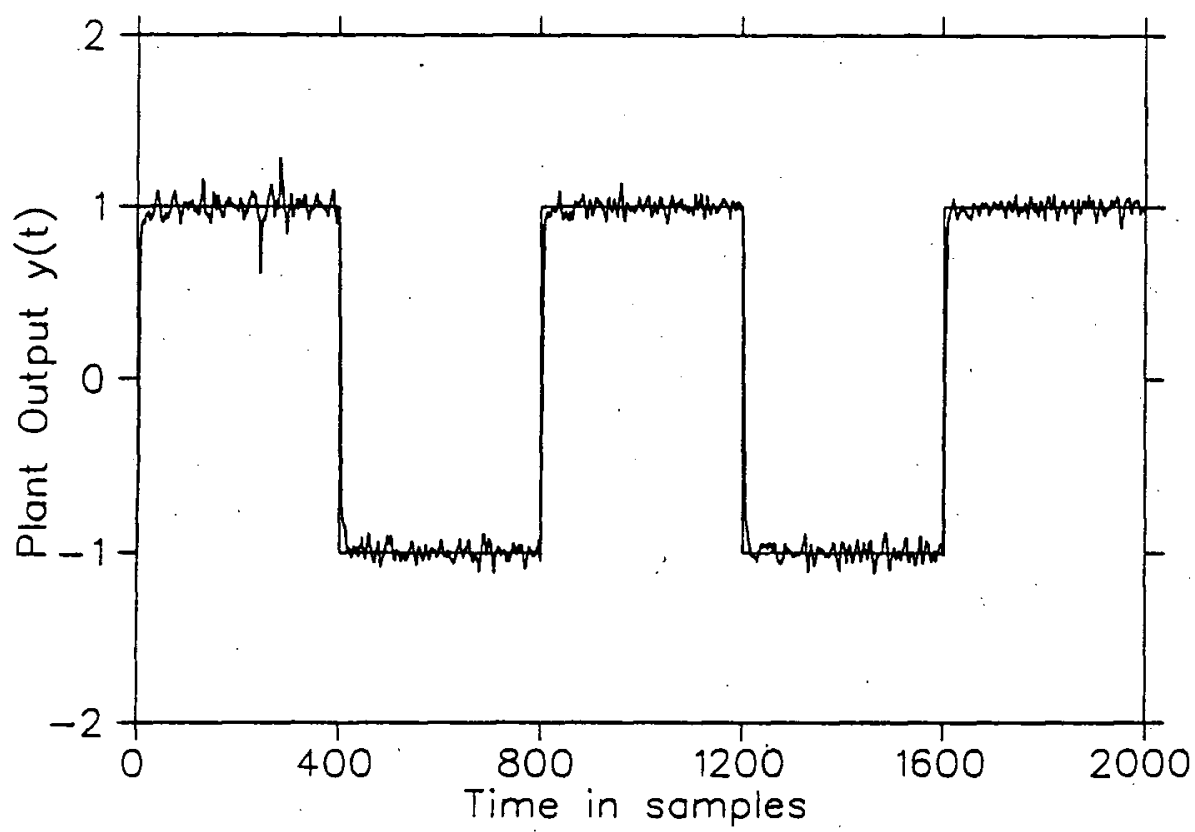

Figure 4.29: Example 4.1: Explicit stochastic self-tuner. System output tracking a square wave set-point. Case 4 of Table 4.2 . 


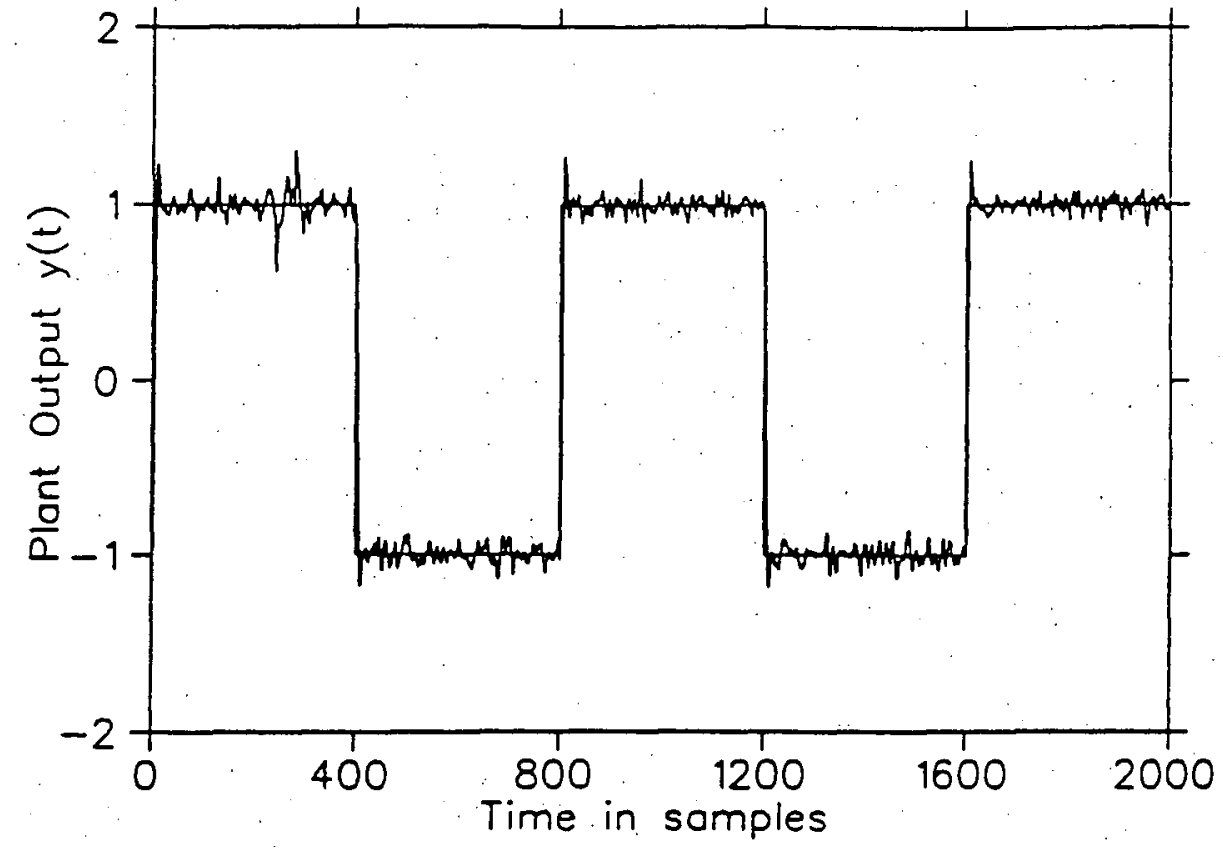

Figure 4.30: Example 4.1: Explicit stochastic self-tuner. System output tracking a square wave set-point. Case 5 of Table 4.2 .

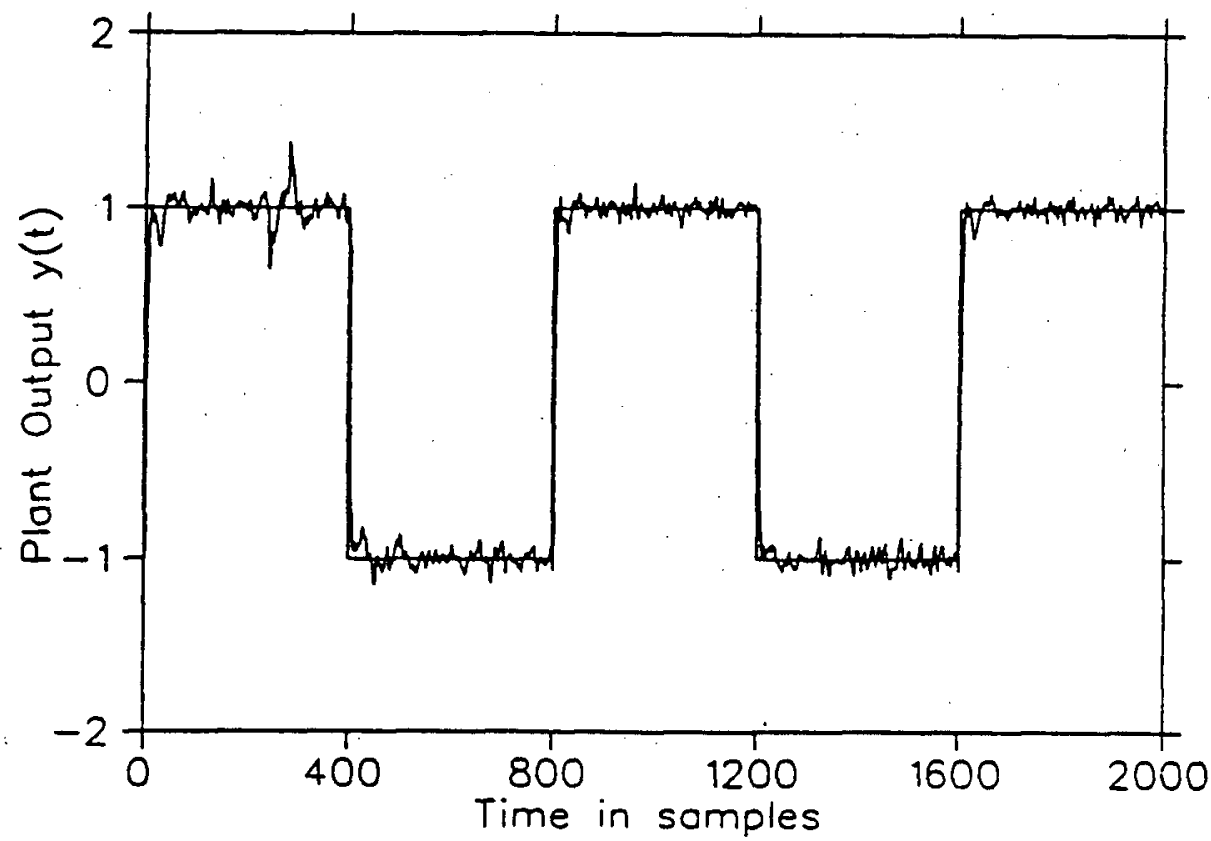

Figure 4.31: Example 4.1: Explicit stochastic self-tuner. System output tracking a square wave set-point. Case 6 of Table 4.2 . 


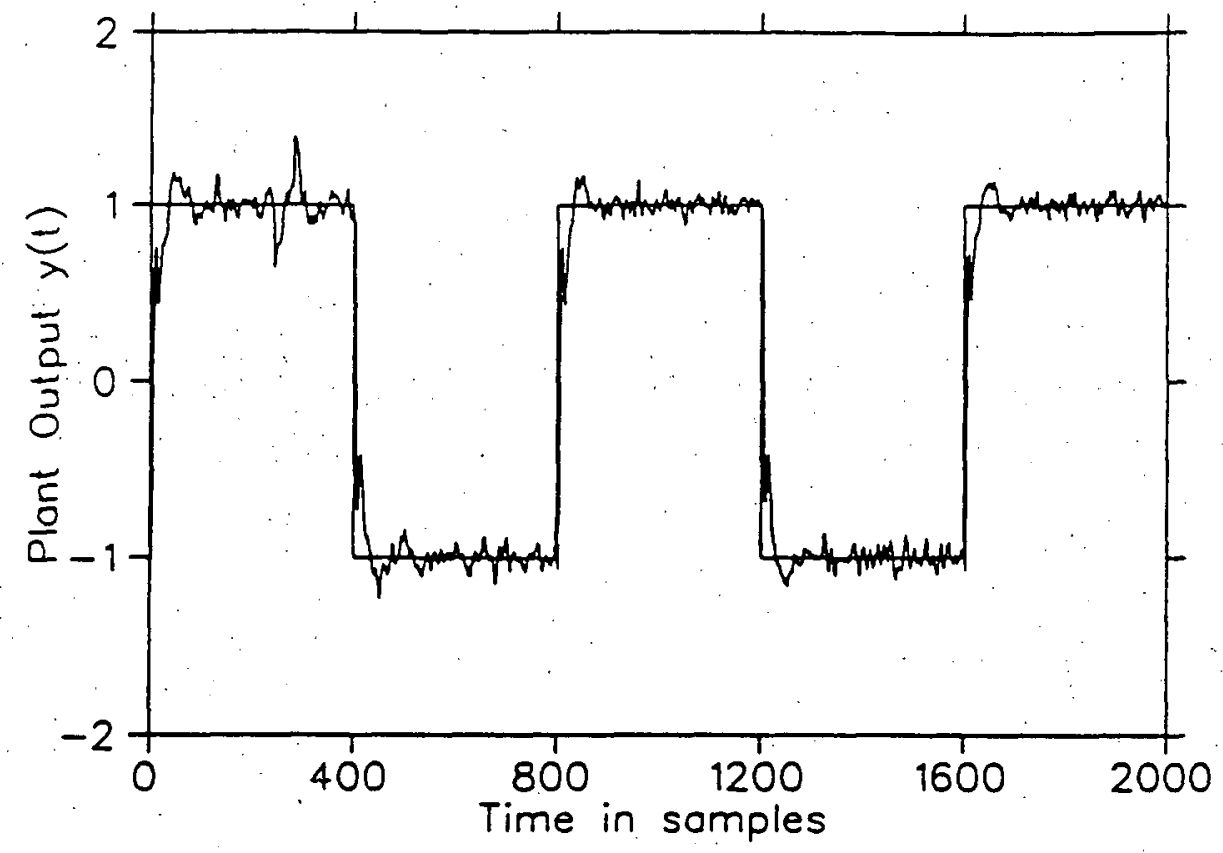

Figure 4.32: Example 4.1: Explicit stochastic self-tuner. System output tracking a square wave set-point. Case 7 of Table 4.2 .

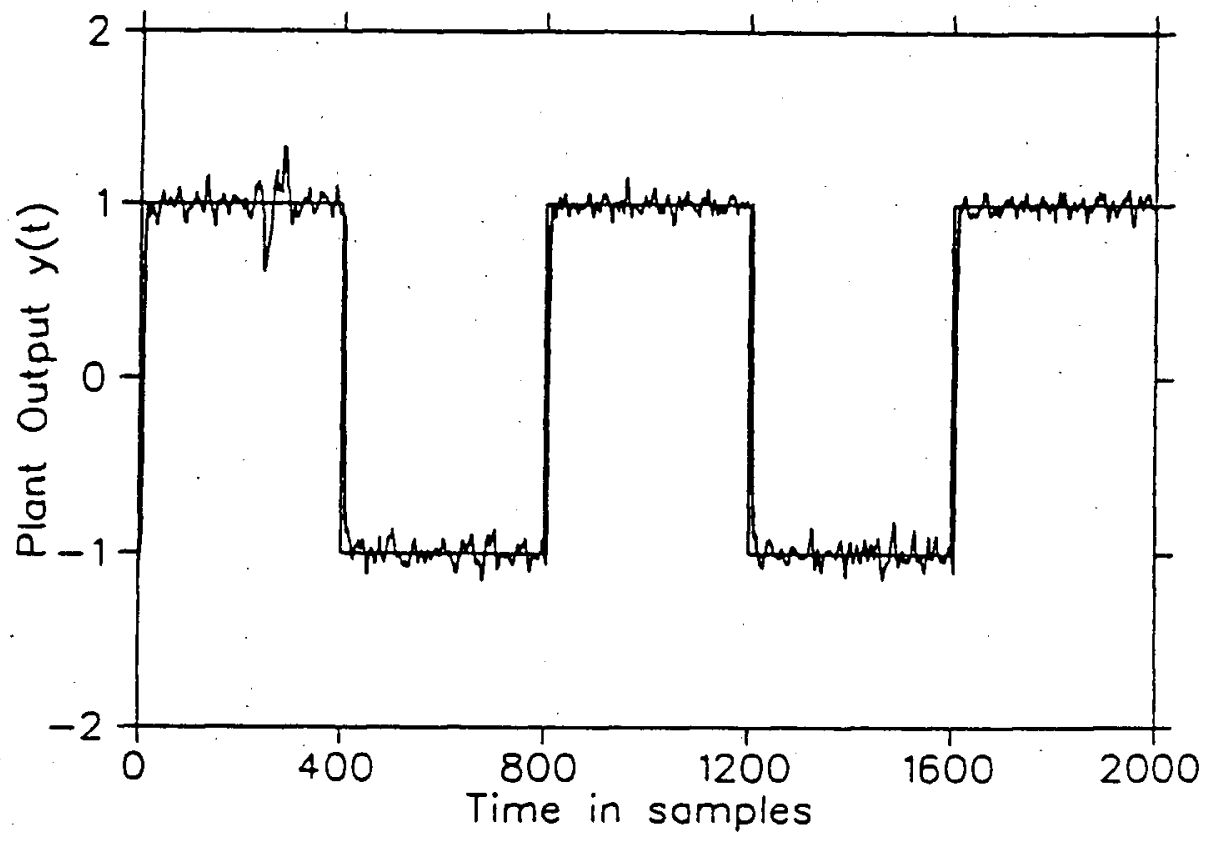

Figure 4.33: Example 4.1: Explicit stochastic self-tuner. System output tracking a square wave set-point. Case 8 of Table 4.2 . 


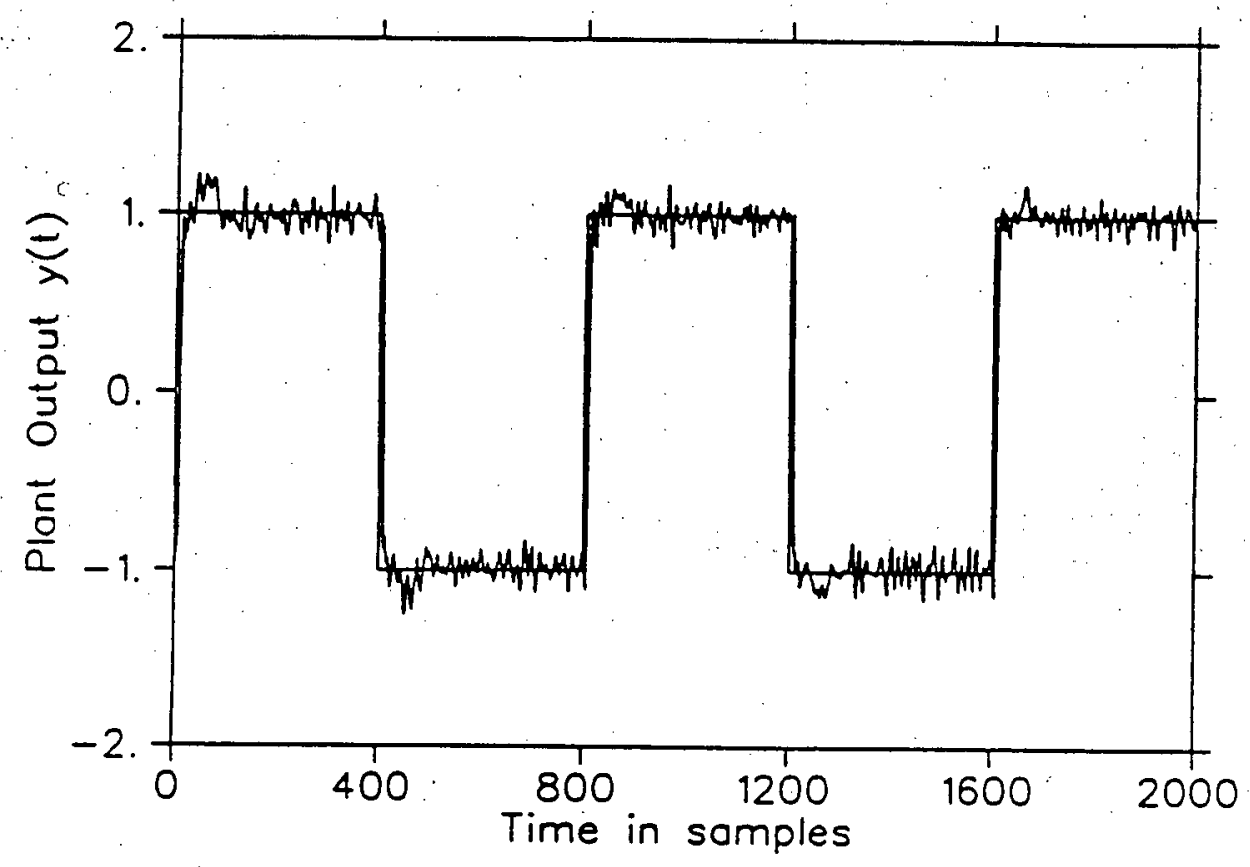

Figure 4.34: Example 4.1: Implicit stochastic self-tuner. System output tracking a square wave set-point. 


\section{Chapter 5}

\section{Multivariable Self-Tuning Control}

\subsection{Introduction}

The basic thrust of the work presented so far in this thesis has been the development of adaptive control schemes based on orthonormal series representation, with the advantage of reduced a-priori knowledge, easy handling of unknown and varying time delays and non-minimum phase plants, and increased robustness in presence of unmodeled dynamics. Up to now, only single-input, single-output plants (SISO) have been considered. However it is straightforward to extend the adaptive controllers based on Laguerre functions previously developed (Dumont and Zervos, 1986; Zervos and Dumont, 1988a; Zervos and Dumont, 1988b) to the multivariable case.

The most challenging problem in this area has been the representation of time-delays of MIMO systems via a delay matrix (Mohtadi, Shah, Clarke and 1987). The delay matrix of a MIMO system is the direct generalization of the time-delay term associated with a SISO system. This matrix characterizes the infinite zeros of the MIMO process but does not have a unique structure (Elliott and Wolovich, 1984). One particular lower triangular form of the delay matrix as defined by Wolovich and Falb (1976) is known as the interactor matrix. Many early results in MIMO adaptive control considered special classes of this delay matrix : a diagonal matrix and others required prior knowledge of the triangular interactor matrix. However, the use of orthonormal functions in adaptive control can be extended to the MIMO case so that the requirement for knowing the 


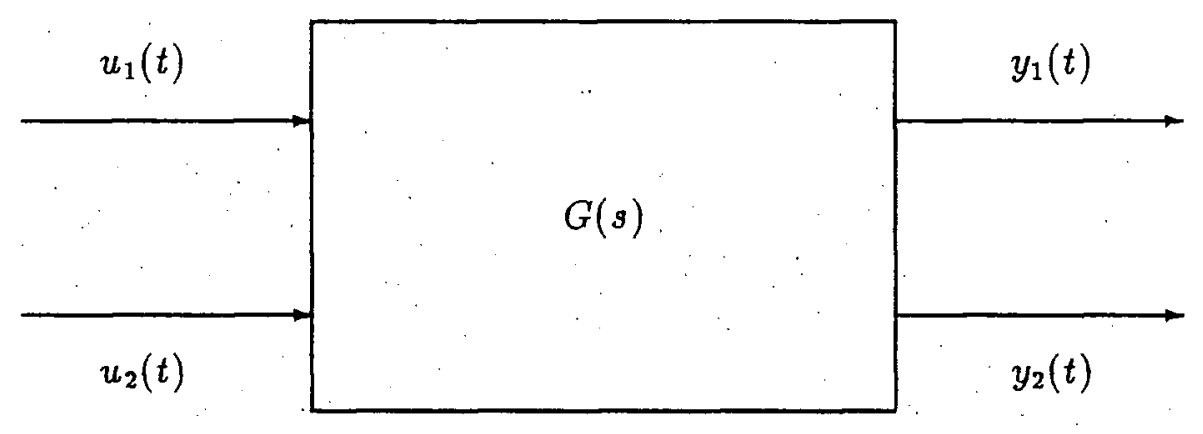

Figure 5.35: Multivariable $2 \times 2$ input-output linear system.

system delay matrix is completely avoided. As a result, all the problems associated with the definition of the interactor matrix are completely eliminated. This is considered to be a definite advantage in the formulation of a multivariable adaptive scheme because it adds simplicity to the design and representation of the algorithm.

\subsection{Deterministic Multivariable Self-Tuning Control}

The development of the deterministic multivariable self-tuning controller is a direct extension of the SISO equivalent controller described in details in Section 3.1. What follows is an illustrative mathematical derivation and two simulation examples that show how the principles outlined so far in this thesis can be used in modeling a multivariable linear system. Let us consider, for simplicity, the 2-input, 2-output linear plant $G(s)$ shown in Figure 5.35, typical of a paper machine headbox, where, $u_{1}(t)$, $u_{2}(t)$ are the plant inputs and $y_{1}(t), y_{2}(t)$ are the plant outputs. This system can be modeled by the Laguerre ladder network shown in Figure 5.36. The same principles would however apply for any $(N \times N)$ configuration.

The following equations can describe the model equations in state-space form. Two 


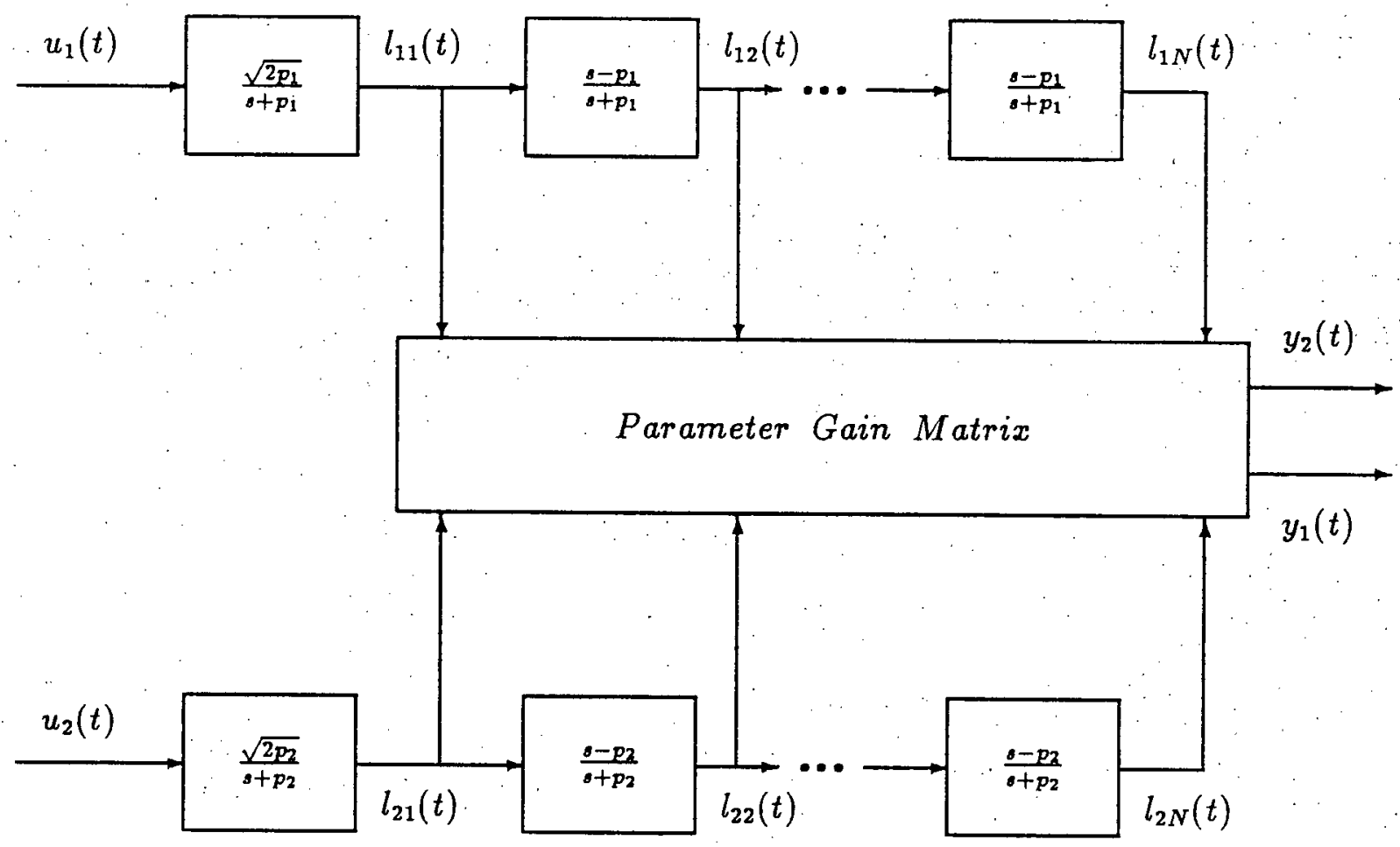

Figure 5.36: Laguerre Ladder Network for a Multivariable $2 \times 2$ input-output linear system. 
different sets of Laguerre orthonormal functions have been employed, one with timescale $p_{1}$ and the other one with time-scale $p_{2}$. For the sake of simplicity let also assume that both sets have the same number $N$ of functions. The first set can be described by the state-space description,

$$
\underline{L}_{1}(t+1)=A_{1} \underline{L}_{1}(t)+\underline{b}_{1} u_{1}(t)
$$

and the second set by,

$$
\underline{L}_{2}(t+1)=A_{2} \underline{L}_{2}(t)+\underline{b}_{2} u_{2}(t)
$$

where,

$$
\begin{aligned}
& \underline{L}_{1}=\left[\begin{array}{llll}
l_{12} & l_{12} & \cdots & l_{1 N}
\end{array}\right]^{T} \\
& \underline{L}_{2}=\left[\begin{array}{llll}
l_{21} & l_{22} & \cdots & l_{2 N}
\end{array}\right]^{T}
\end{aligned}
$$

If we define the combined state vector to be,

$$
\underline{L}^{T}=\left[\begin{array}{ll}
\underline{L}_{1}^{T} & \underline{L}_{2}^{T}
\end{array}\right]
$$

and the output vector to be,

$$
\underline{Y}=\left[\begin{array}{ll}
y_{1} & y_{2}
\end{array}\right]^{T}
$$

then we can write,

$$
\underline{Y}(t)=C \underline{L}(t)
$$

Where the parameter gain matrix $C$ is of the form,

$$
C=\left|\begin{array}{ll}
\underline{C}_{11}^{T} & \underline{C}_{12}^{T} \\
\underline{C}_{21}^{T} & \underline{C}_{22}^{T}
\end{array}\right|=\left|\begin{array}{cccccccc}
c_{11}^{1} & c_{12}^{1} & \ldots & c_{1 N}^{1} & c_{21}^{1} & c_{22}^{1} & \ldots & c_{2 N}^{1} \\
c_{11}^{2} & c_{12}^{2} & \ldots & c_{1 N}^{2} & c_{21}^{2} & c_{22}^{2} & \ldots & c_{2 N}^{2}
\end{array}\right|
$$

where we used the notation,

$$
\underline{C}_{i j}^{T}=\left[\begin{array}{llll}
c_{j 1}^{i} & c_{j 2}^{i} & \ldots & c_{j N}^{i}
\end{array}\right]
$$


and $i, j$ are both indices.

For the structure of the lower triangular matrices $A_{1}$ and $A_{2}$, and the input vectors $\underline{b}_{1}$ and $\underline{b}_{2}$ see Section 2.3. The parameter gain matrix $C$ can be estimated on-line by a multivariable least-squares method. In an analogous way as outlined in Section 3.1 we can derive the predictive expressions for the state vectors and for the model plant outputs. Assuming,

$$
\begin{aligned}
& u_{1}(t)=u_{1}(t+1)=\cdots=u_{1}\left(t+d_{1}-1\right) \\
& u_{2}(t)=u_{2}(t+1)=\cdots=u_{2}\left(t+d_{2}-1\right)
\end{aligned}
$$

we can write,

$$
\begin{aligned}
& \underline{L}_{1}\left(t+d_{1}\right)=A_{1}^{d_{1}} \underline{L}_{1}(t)+\left(A_{1}^{d_{1}-1}+\cdots+I\right) \underline{b}_{1} u_{1}(t) \\
& L_{2}\left(t+d_{2}\right)=A_{2}^{d_{2}} \underline{L}_{2}(t)+\left(A_{2}^{d_{2}-1}+\cdots+I\right) \underline{b}_{2} u_{2}(t)
\end{aligned}
$$

where $d_{1}$ and $d_{2}$ are the prediction horizons. Then the required predictive output expression for $y_{1}(t)$ is,

$$
\begin{aligned}
y_{1}\left(t+d_{1}\right)= & \underline{C}_{11}^{T}\left(A_{1}^{d_{1}}-I\right) \underline{L}_{1}(t)+\underline{C}_{12}^{T}\left(A_{2}^{d_{1}}-I\right) \underline{L}_{2}(t) \\
& +\underline{C}_{11}^{T}\left(A_{1}^{d_{1}-1}+\cdots+I\right) \underline{b}_{1} u_{1}(t) \\
& \left.+\underline{C}_{12}^{T}\left(A_{2}^{d_{1}-1}+\cdots+I\right) \underline{b}_{2} u_{2}(t)+y_{1}(t)\right)
\end{aligned}
$$

If we define,

$$
\begin{gathered}
\underline{P}_{11}^{T}=\underline{C}_{11}^{T}\left(A_{1}^{d_{1}}-I\right) \\
\underline{P}_{12}^{T}=\underline{C}_{12}^{T}\left(A_{2}^{d_{1}}-I\right) \\
B_{11}=\underline{C}_{11}^{T}\left(A_{1}^{d_{1}-1}+\cdots+I\right) \underline{b}_{1} \\
B_{12}=\underline{C}_{12}^{T}\left(A_{2}^{d_{1}-1}+\cdots+I\right) \underline{b}_{2}
\end{gathered}
$$


Then equation (5.179) becomes,

$$
\left.y_{1}\left(t+d_{1}\right)=\underline{P}_{11}^{T} \underline{L}_{1}(t)+\underline{P}_{12}^{T} \underline{L}_{2}(t)+B_{11} u_{1}(t)+B_{12} u_{2}(t)+y_{1}(t)\right)
$$

In the same way we can derive the predictive expression for the output $y_{2}(t)$ to be,

$$
\left.y_{2}\left(t+d_{2}\right)=\underline{P}_{21}^{T} \underline{L}_{1}(t)+\underline{P}_{22}^{T} \underline{L}_{2}(t)+B_{21} u_{1}(t)+B_{22} u_{2}(t)+y_{2}(t)\right)
$$

where,

$$
\begin{gathered}
\underline{P}_{21}^{T}=C_{21}^{T}\left(A_{1}^{d_{2}}-I\right) \\
\underline{P}_{22}^{T}=\underline{C}_{22}^{T}\left(A_{2}^{d_{2}}-I\right) \\
B_{21}=\underline{C}_{21}^{T}\left(A_{1}^{d_{2}-1}+\cdots+I\right) \underline{b}_{1} \\
B_{22}=\underline{C}_{22}^{T}\left(A_{2}^{d_{2}-1}+\cdots+I\right) \underline{b}_{2}
\end{gathered}
$$

Now if we define two reference trajectories $y_{r 1}$ and $y_{r 2}$, in the same way as we did in Section 3.1. and equate them to the r.h.s of equations $(5.180),(5.181)$ respectively, we get a system of 2 equations with 2 unknowns, $u_{1}(t), u_{2}(t)$.

$$
\left\{\begin{array}{l}
B_{11} u_{1}(t)+B_{12} u_{2}(t)=y_{r 1}-y_{1}(t)-\underline{P}_{11}^{T} \underline{L}_{1}(t)-\underline{P}_{12}^{T} \underline{L}_{2}(t) \\
B_{21} u_{1}(t)+B_{22} u_{2}(t)=y_{r 2}-y_{2}(t)-\underline{P}_{21}^{T} \underline{L}_{1}(t)-\underline{P}_{22}^{T} \underline{L}_{2}(t)
\end{array}\right\}
$$

Defining,

$$
\begin{aligned}
& D_{1}=y_{r 1}-y_{1}(t)-\underline{P}_{11}^{T} \underline{L}_{1}(t)-\underline{P}_{12}^{T} \underline{L}_{2}(t) \\
& D_{2}=y_{r 2}-y_{2}(t)-\underline{P}_{21}^{T} \underline{L}_{1}(t)-\underline{P}_{22}^{T} \underline{L}_{2}(t)
\end{aligned}
$$

then the above system (equation (5.182)) can be solved for the unknowns $u_{1}(t), u_{2}(t)$ to give in determinant form,

$$
u_{1}(t)=\frac{\left|\begin{array}{ll}
D_{1} & B_{12} \\
D_{2} & B_{22}
\end{array}\right|}{\left|\begin{array}{ll}
B_{11} & B_{12} \\
B_{21} & B_{22}
\end{array}\right|} \quad, \quad u_{2}(t)=\frac{\left|\begin{array}{ll}
B_{11} & D_{1} \\
B_{21} & D_{2}
\end{array}\right|}{\left|\begin{array}{ll}
B_{11} & B_{12} \\
B_{21} & B_{22}
\end{array}\right|}
$$




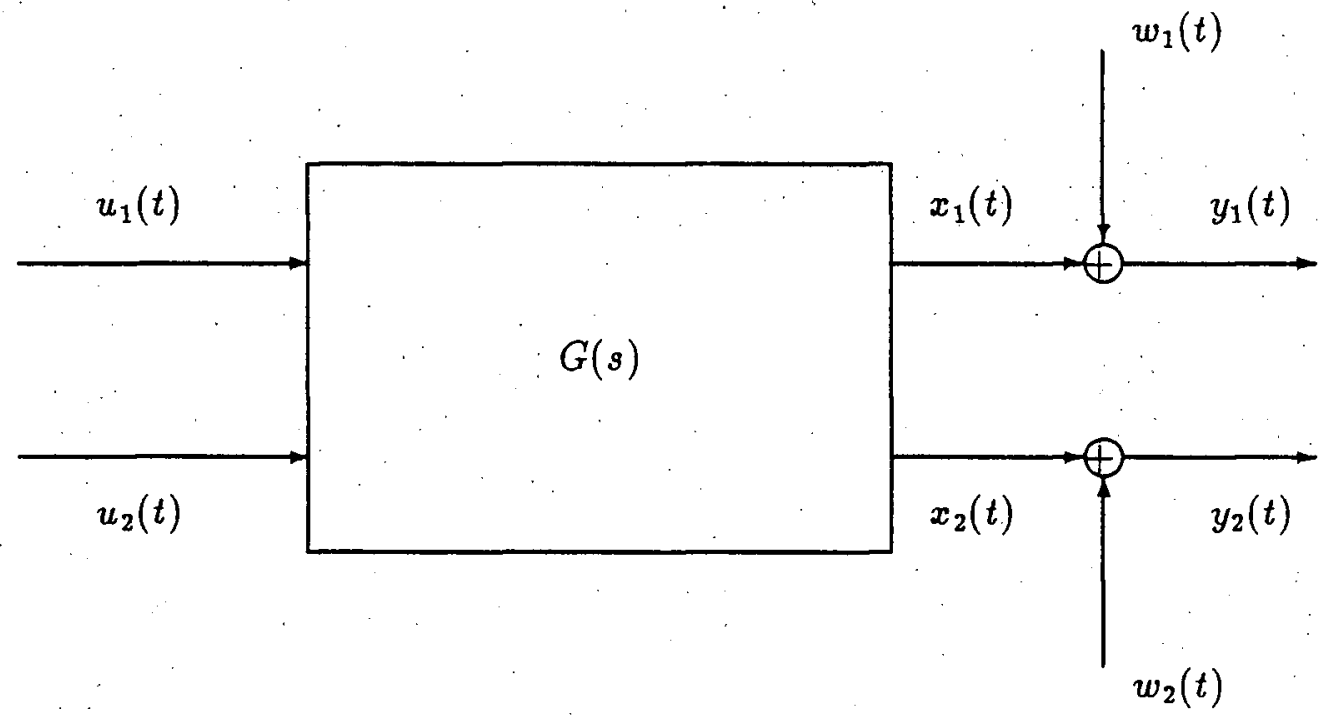

Figure 5:37: Stochastic multivariable $2 \times 2$ linear system.

And these are the required control inputs to form the deterministic multivariable selftuning scheme.

\subsection{Stochastic Multivariable Self-Tuning Control}

The same principles outlined in Section 4.2 for the single-input, single-output case can also be applied to derive a stochastic multivariable scheme based on the Laguerre orthonormal functions. Let us consider again for simplicity a $2 \times 2$ linear stochastic multivariable system as shown in Figure 5.3\%. Where $w_{1}(t)$ and $w_{2}(t)$ are stochastic bounded noise sequences.

Following the ideas outlined in sections 4.2 and 5.2 we can write for the deterministic 
part of the plant,

$$
\begin{aligned}
& \underline{L}_{1}(t+1)=A_{1} \underline{L}_{1}(t)+\underline{b}_{1} u_{1}(t) \\
& \underline{L}_{2}(t+1)=A_{2} \underline{L}_{2}(t)+\underline{b}_{2} u_{2}(t) \\
& {\left[\begin{array}{l}
x_{1} \\
x_{2}
\end{array}\right]=\left[\begin{array}{ll}
\underline{C}_{11}^{T} & C_{12}^{T} \\
\underline{C}_{21}^{T} & C_{22}^{T}
\end{array}\right]\left[\begin{array}{l}
\underline{L}_{1} \\
\underline{L}_{2}
\end{array}\right]}
\end{aligned}
$$

where the notation of Section 5.2 and of the Figure 5.37 was followed.

The stochastic part of the plant can be expressed in an analogous way by the equations,

$$
\begin{gathered}
\underline{N}_{1}(t+1)=A_{1}^{\prime} \underline{N}_{1}(t)+\underline{b}_{1}^{\prime} e_{1}(t) \\
w_{1}(t)=\underline{C}_{1}^{\prime T} \underline{N}_{1}(t)+e_{1}(t)
\end{gathered}
$$

and,

$$
\begin{gathered}
\underline{N}_{2}(t+1)=A_{2}^{\prime} \underline{N}_{2}(t)+\underline{b}_{2}^{\prime} e_{2}(t) \\
w_{2}(t)=\underline{C}_{2}^{\prime T} \underline{N}_{2}(t)+e_{2}(t)
\end{gathered}
$$

where the notation of Section 4.2 was followed and $\underline{N}_{i}=\left[\begin{array}{lll}n_{i 1} & n_{i 2} \ldots n_{i N_{i}^{\prime}}\end{array}\right]$ with $i=$ 1,2 , is respectively the noise state-vector for each network representation. The noise sequences $e_{1}(t)$ and $e_{2}(t)$ are normal white noises $N(0,1)$. Predictive expressions can also be derived for the outputs $y_{1}(t)$ and $y_{2}(t)$ as was described in Sections 4.2 and 5.2, that is, using equations, $(5.187),(5.189), y_{1}(t)$ can be written as,

$$
\begin{aligned}
y_{1}\left(t+d_{1}\right)= & \underline{C}_{11}^{T}\left(A_{1}^{d_{1}}-I\right) \underline{L}_{1}(t)+\underline{C}_{12}^{T}\left(A_{2}^{d_{1}}-I\right) \underline{L}_{2}(t) \\
& +\underline{C}_{11}^{T}\left(A_{1}^{d_{1}-1}+\cdots+I\right) \underline{b}_{1} u_{1}(t)+\underline{C}_{12}^{T}\left(A_{2}^{d_{1}-1}+\cdots+I\right) \underline{b}_{2} u_{2}(t) \\
& +\underline{C}_{1}^{\prime T}\left(A_{1}^{\prime d_{1}}-I\right) \underline{N}_{1}(t)+\underline{C}_{1}^{\prime T}\left(A_{1}^{\prime d_{1}-1} \underline{b}_{1}^{\prime}(t) e_{1}(t)+\cdots+\underline{b}_{1}^{\prime}(t) e_{1}\left(t+d_{1}+1\right)\right) \\
& +e_{1}\left(t+d_{1}\right)-e_{1}(t)+y_{1}(t)
\end{aligned}
$$

If we define,

$$
\underline{P}_{11}^{T}=\underline{C}_{11}^{T}\left(A_{1}^{d_{1}}-I\right)
$$




$$
\begin{gathered}
\underline{P}_{12}^{T}=\underline{C}_{12}^{T}\left(A_{2}^{d_{1}}-I\right) \\
B_{11}=\underline{C}_{11}^{T}\left(A_{1}^{d_{1}-1}+\cdots+I\right) \underline{b}_{1} \\
B_{12}=\underline{C}_{12}^{T}\left(A_{2}^{d_{1}-1}+\cdots+I\right) \underline{b}_{2} \\
\underline{Q}_{1}^{T}=\underline{C}_{1}^{\prime T}\left(A_{1}^{\prime d_{1}}-I\right) \\
\underline{Q}_{1}^{\prime T}=\underline{C}_{1}^{T}\left(A_{1}^{\prime d_{1}-1} \underline{b}_{1}^{\prime}(t)-1\right)
\end{gathered}
$$

Then the best prediction of $y_{1}\left(t+d_{1} \mid t\right)$ is given by,

$$
\begin{aligned}
y_{1}\left(t+d_{1} \mid t\right)= & \underline{P}_{11}^{T} \underline{L}_{1}(t)+\underline{P}_{12}^{T} \underline{L}_{2}(t)+B_{11} u_{1}(t)+B_{12} u_{2}(t) \\
& +\underline{Q}_{1}^{T} \underline{N}_{1}(t)+\underline{Q}_{1}^{T} \hat{e}_{1}(t)+y_{1}(t)
\end{aligned}
$$

where $\hat{e}_{1}(t)$ is the proxying of the estimated noise $e_{1}(t)$ and can be calculated in the same way as it was described in Section 4.2. In a similar way the best predictive expression for $y_{2}(t)$ can be obtained.

$$
\begin{aligned}
y_{2}\left(t+d_{2} \mid t\right)= & \underline{P}_{21}^{T} \underline{L}_{1}(t)+\underline{P}_{22}^{T} \underline{L}_{2}(t)+B_{21} u_{1}(t)+B_{22} u_{2}(t) \\
& +\underline{Q}_{2}^{T} \underline{N}_{2}(t)+\underline{Q}_{2}^{T} \hat{e}_{2}(t)+y_{2}(t)
\end{aligned}
$$

where,

$$
\begin{gathered}
\underline{P}_{21}^{T}=\underline{C}_{21}^{T}\left(A_{1}^{d_{2}}-I\right) \\
\underline{P}_{22}^{T}=\underline{C}_{22}^{T}\left(A_{2}^{d_{2}}-I\right) \\
B_{21}=\underline{C}_{21}^{T}\left(A_{1}^{d_{2}-1}+\cdots+I\right) \underline{b}_{1} \\
B_{22}=\underline{C}_{22}^{T}\left(A_{2}^{d_{2}-1}+\cdots+I\right) \underline{b}_{2} \\
\underline{Q}_{2}^{T}=\underline{C}_{2}^{\prime T}\left(A_{2}^{\prime d_{2}}-I\right) \\
\underline{Q}_{2}^{i T}=\underline{C}_{2}^{T}\left(A_{2}^{\prime d_{1}-1} \underline{b}_{2}^{\prime}(t)-1\right)
\end{gathered}
$$


If two reference trajectories are defined, $y_{r 1}$ and $y_{r 2}$, and equate them to the r.h.s. parts of equations (5.192) and (5.193) respectively, a system of 2 equations with 2 unknowns, $u_{1}(t)$ and $u_{2}(t)$, is formed. To solve for the required control inputs, the method described in Section 5.2 can be adopted.

\subsection{Simulation examples}

For illustrative purposes two examples follow that use the deterministic multivariable self-tuning scheme, described earlier, in a stochastic environment. The first one controls a simple second-order multivariable $2 \times 2$ linear plant. The second discusses how the same self-tuning scheme can be used to control the headbox of a paper machine. So many researchers have simulated this process in the past using various self-tuning regulators that it has become a benchmark for testing multivariable adaptive control schemes.

Example 5.1: Consider the $2 \times 2$ second-order linear stochastic multivariable plant,

$$
\begin{aligned}
y_{1}(t)= & 0.2 y_{1}(t-1)+0.1 y_{1}(t-2)+u_{1}(t-1) \\
& +0.8 u_{1}(t-2)+u_{2}(t-2)+w_{1}(t) \\
y_{2}(t)= & 0.2 y_{2}(t-1)+0.1 y_{2}(t-2)+u_{1}(t-1) \\
& +0.1 u_{1}(t-2)+u_{2}(t-1)+w_{2}(t)
\end{aligned}
$$

where the covariance matrix $W$ of the disturbances is given by,

$$
W=\left[\begin{array}{cc}
0.2 & 0.35 \\
0.35 & 0.6
\end{array}\right]
$$

The deterministic multivariable Laguerre self-tuner that was applied to the above plant used, $N_{1}=N_{2}=8$, the Laguerre time-scales $p_{1}=0.1, p_{2}=0.7$, with prediction horizons 


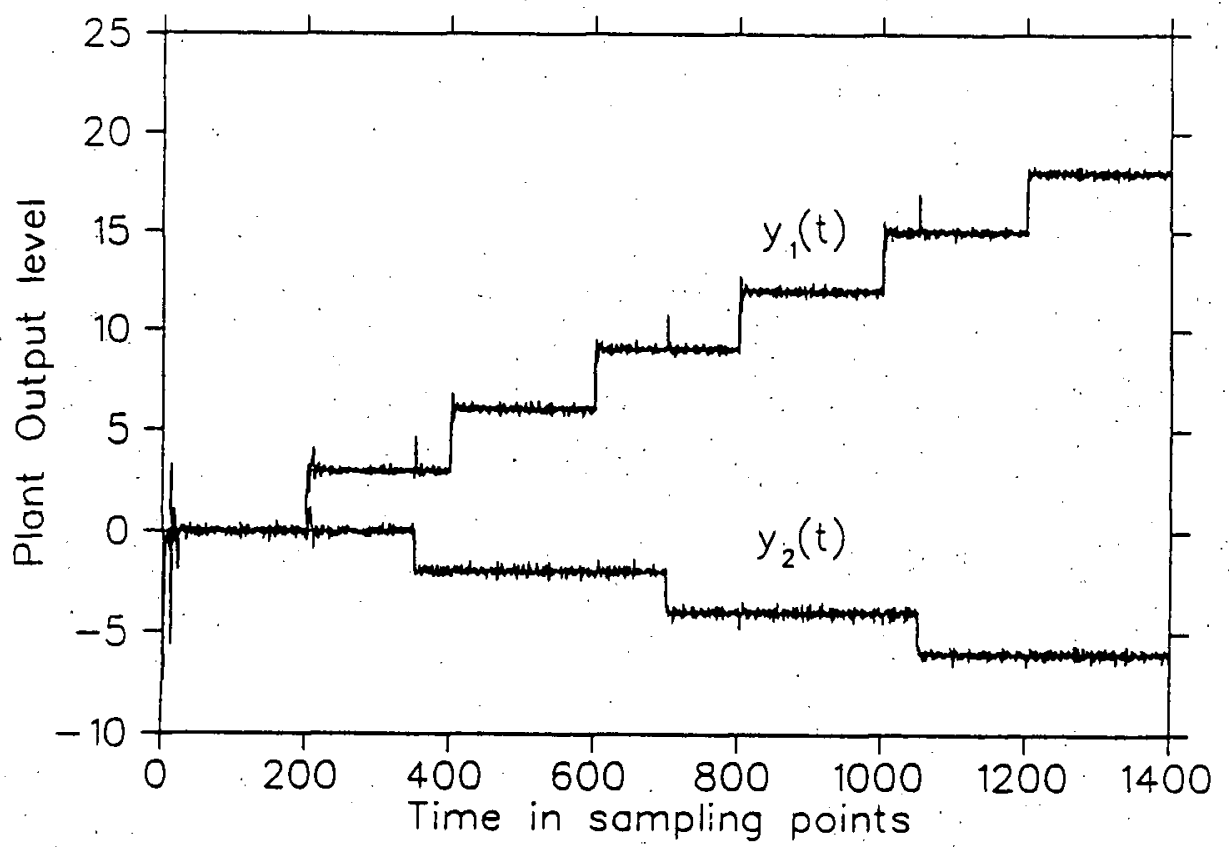

Figure 5.38: Example 5.1: Explicit multivariable self-tuning control of a $2 \times 2$ plant. System outputs tracking two respective reference trajectories.

$d_{1}=2, d_{2}=2$, and the driver block filter time constants $\alpha_{1}=\alpha_{2}=0.01$. All the initial parameter estimates were zero. The initial covariance matrices for the multivariable RLS algorithm were $1000 \times I$, and all the forgetting factors were 1 . The simulation run was performed over 1400 points starting from zero reference level for both outputs. The set-point for the first output was an upward staircase-like trajectory with step jump of +3 every 200 points. The set-point of the second output was a downward staircase-like trajectory with step jump of -2 every 350 points. Figure 5.38 shows the two plant outputs following their respective reference trajectories. Figure 5.39 shows the behaviour of the multivariable adaptive controller outputs for the same simulation run.

Example 5.2: The headbox is a vital and very important section of a papermachine. Figure 5.40 gives a schematic description of a headbox. Its purpose is to change the turbulent flow in the lube going into the headbox to a sheet flow out of the 


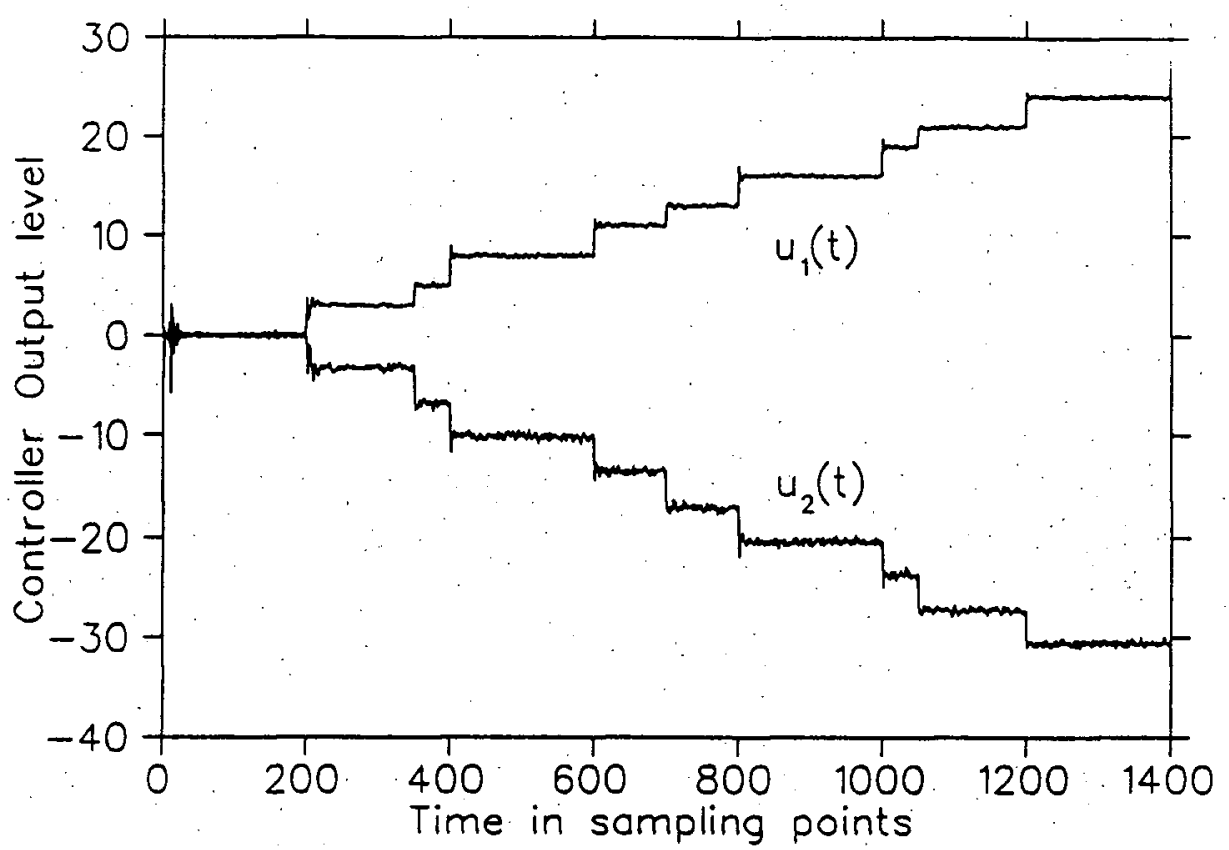

Figure 5.39: Example 5.1: Explicit multivariable self-tuning control of a $2 \times 2$ plant. Controller outputs.

headbox. The operation of the headbox has a significant effect on the characteristics of the produced paper. The headbox and its associated flow system is a complicated hydro-dynamical device. The most.important control problem for a headbox is to maintain constant jet velocity and to have a good dynamic behaviour when changing the headbox level. The variations are changes in stockflow and airflow into the headbox. In the present study it is assumed that the air cushion of the headbox is pressurized using a compressor and that the airflow is manipulated either through the massflow into the headbox or through a valve at the outlet $(q(t)$ in the Figure 5.40$)$.

The control of a paper-machine headbox is a good example of an interactive multivariable control system. The mathematical model equations derived from physical principles show the system to be nonlinear of second order and the obtained differential equations fall into the category of stiff systems. However, for small perturbations around a steady state operating point the process can be considered linear ( $\AA$ ström, 


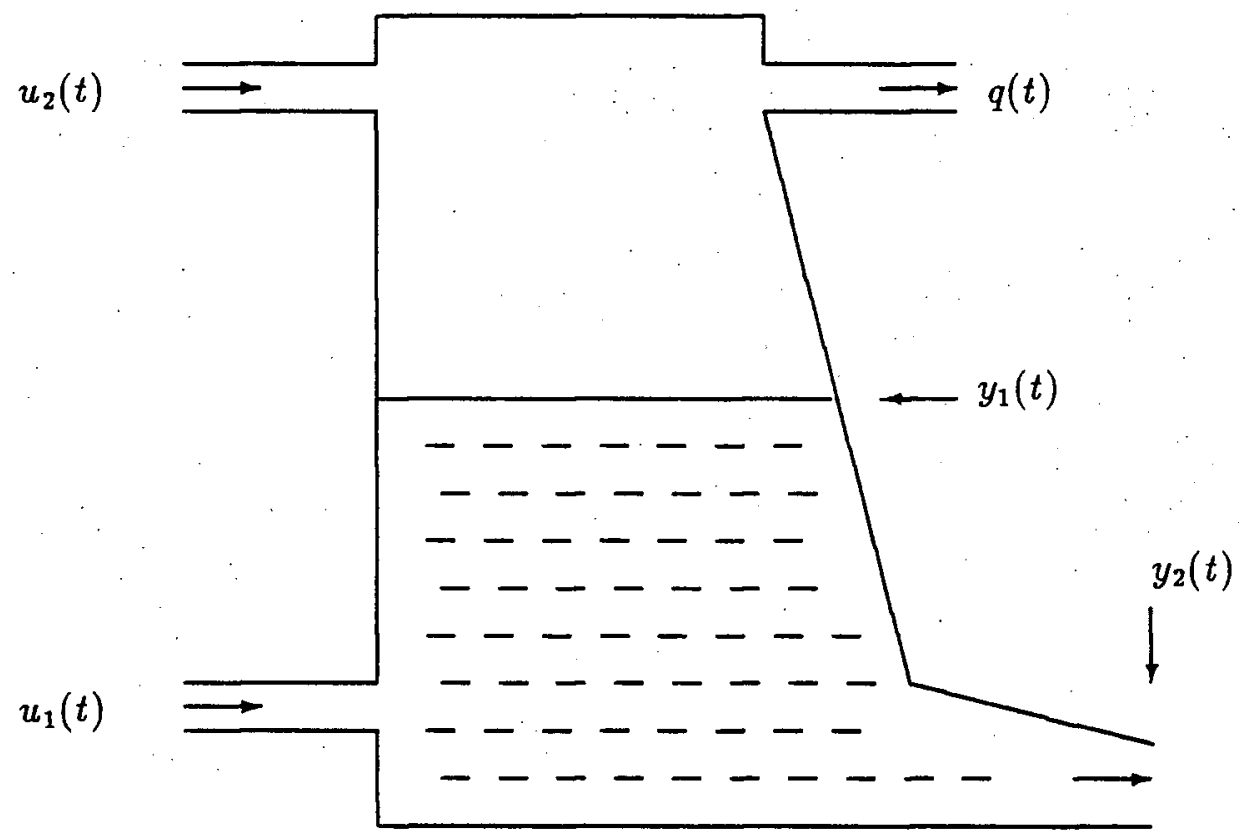

Figure 5.40: Example 5.2: Headbox of a paper-machine. 
1972). Following the principles and the headbox prototype model specifications outlined in Aström (1972) and Natarajan (1988) the continuous state-space linearized model can be derived in the form,

$$
\begin{aligned}
\underline{x}(t) & =\left[\begin{array}{cc}
-0.0115 & -0.1411 \\
-0.0373 & -0.5270
\end{array}\right] \underline{x}(t)+\left[\begin{array}{cc}
0.1 & 0.0 \\
0.324 & 0.2
\end{array}\right] \underline{u}(t) \\
\underline{y}(t) & =\left[\begin{array}{cc}
1.0 & 0.0 \\
1.0 & 12.2412
\end{array}\right] \underline{x}(t)
\end{aligned}
$$

where,

$$
\begin{aligned}
& y_{1}(t) \text { is the stock level } \\
& y_{2}(t) \text { is the total head pressure } \\
& x_{1}(t) \text { is the stock level } \\
& x_{2}(t) \text { is the air pad density } \\
& u_{1}(t) \text { is the stock volume flow rate } \\
& u_{2}(t) \text { is the air mass flow rate }
\end{aligned}
$$

and the operating point was chosen to be,

$$
\begin{aligned}
& y_{1}(t)=0.5 \mathrm{~m} \\
& y_{2}(t)=4.3339 \mathrm{~m} \mathrm{H}_{2} \mathrm{O} \\
& x_{1}(t)=0.5 \mathrm{~m} \\
& x_{2}(t)=1.62 \mathrm{~kg} / \mathrm{m}^{3} \\
& u_{1}(t)=1.0 \mathrm{~m}^{3} / \mathrm{sec} \\
& u_{2}(t)=0.245 \mathrm{~kg} / \mathrm{sec}
\end{aligned}
$$


This model was transformed to discrete form using a sampling interval of 1 sec. The following result was obtained,

$$
\begin{aligned}
\underline{x}(t+1) & =\left[\begin{array}{cc}
0.9908 & -0.1091 \\
-0.0288 & 0.5922
\end{array}\right] \underline{x}(t)+\left[\begin{array}{ll}
0.0803 & -0.0119 \\
0.2505 & 0.1556
\end{array}\right] \underline{u}(t) \\
\underline{y}(t) & =\left[\begin{array}{cc}
1.0 & 0.0 \\
1.0 & 12.2412
\end{array}\right] \underline{x}(t)
\end{aligned}
$$

Eliminating $\underline{x}(t)$ and adding stochastic disturbances the following model is obtained,

$$
\begin{aligned}
y_{1}(t)= & 1.583 y_{1}(t-1)-0.5836 y_{1}(t-2) \\
+ & 0.0802 u_{1}(t-1)-0.0749 u_{1}(t-2) \\
- & 0.0119 u_{2}(t-1)-0.0099 u_{2}(t-2)+w_{1}(t) \\
y_{2}(t)= & 1.583 y_{2}(t-1)-0.5836 y_{2}(t-2) \\
& +3.1465 u_{1}(t-1)-3.1411 u_{1}(t-2) \\
+ & 1.8927 u_{2}(t-1)-1.81 u_{2}(t-2)+w_{2}(t)
\end{aligned}
$$

where the inputs, state variables and outputs represent now changes on the operating point conditions. To get a value of the covariance matrix of the noise $\underline{w}(t)$ the following assumptions were made in the simulations: (a) A white noise perturbation on either input of the headbox system was taken to have standard error of $10 \%$ of their respective values, and (b) Measurement white noises with standard errors of $10 \%$ on the level and $10 \%$ on the total head pressure value were respectively assumed. Using the above assumptions on the noise disturbances we can solve the linear stochastic difference headbox state-space equations for the steady-state output noise covariance matrix by means of a discre Le Lyapunov equation ( $\AA$ ström, 1970). The solution gives,

$$
W=\mathbf{E}\left[\underline{w}(t) \underline{w}^{T}(t)\right]=\left[\begin{array}{cc}
0.011 & 0.043 \\
0.043 & 0.3127
\end{array}\right]
$$


The deterministic multivariable Laguerre self-tuning scheme was applied to the derived stochastic linearized headbox model as to form a closed-loop system. The following parameters were used : Number of Laguerre functions for each Laguerre network $N_{1}=N_{2}=8$, Laguerre time-scales $p_{1}=0.06$ and $p_{2}=0.6$ respectively, prediction horizons $d_{1}=d_{2}=2$. The time constants of the output reference trajectories were $\alpha_{1}=\alpha_{2}=0.7$. All the initial parameters were zero. For the first 200 secs the system run on its normal operating point (see Figure 5.41 ) in order to allow for the estimation to obtain a model of the system. To test the system, at the $200^{\text {th }} \mathrm{sec}$ the stock level set-point asked for a positive change of $0.3 m$ for the next 200 secs while the total head pressure set-point was kept constant at its normal operating value. At the $400^{\text {th }}$ sec the stock level set-point returned back to its normal initial operating value. At the $600^{\text {th }}$ sec the total head pressure set-point asked for a positive change of $20 \%$ of its operating value for the next 200 secs while the stock level set-point was kept to its normal operating value. Finally at the $800^{\text {th }}$ sec the total head pressure set-point returned back to its normal operating value. The output responses in Figure 5.41 are indicative of the good regulation obtained on both outputs. 

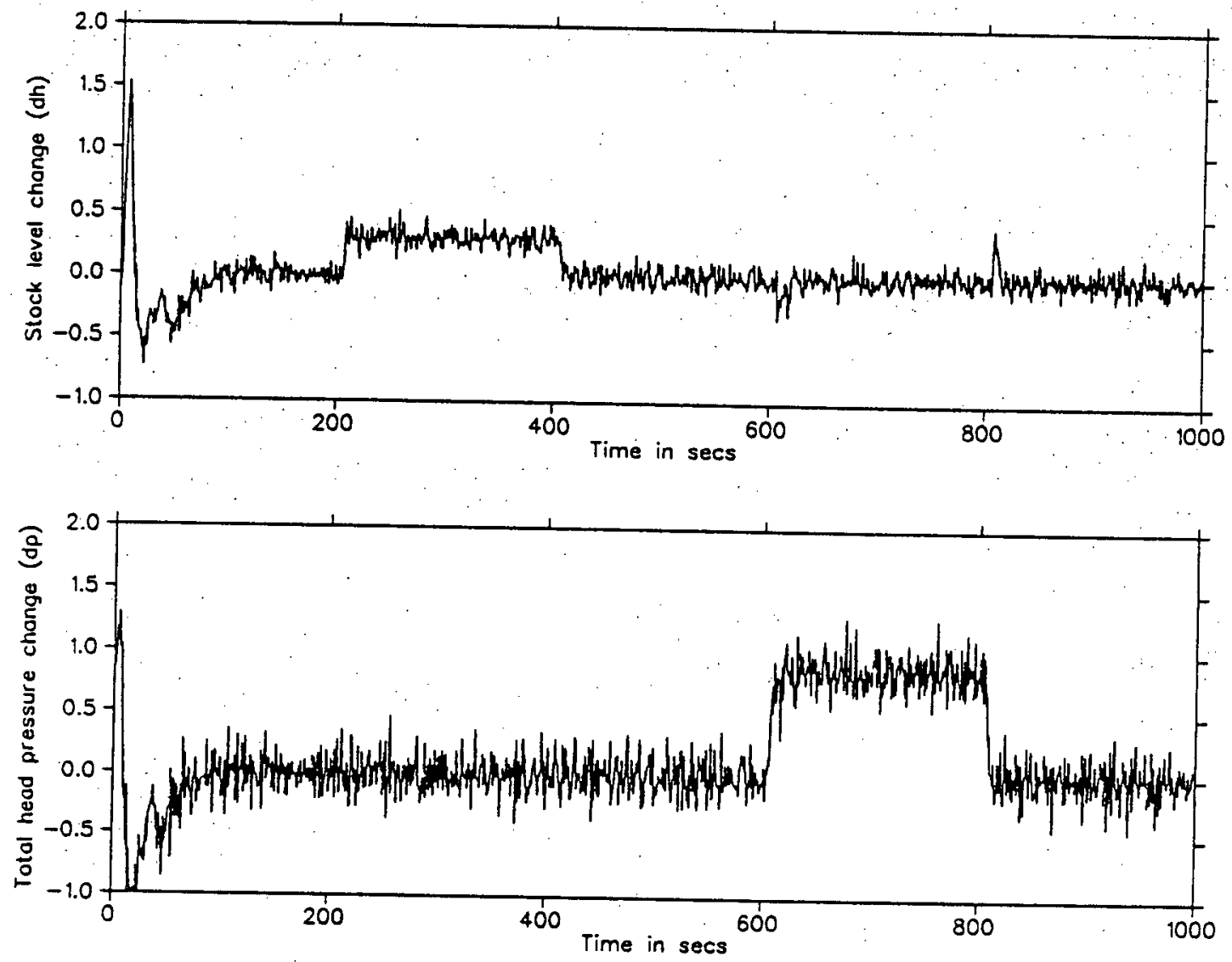

Figure 5.41: Example 5.2: Headbox multivariable self-tuning control. Stock level (upper curve), and Total head pressure (lower) changes: 


\section{Chapter 6}

\section{An Industrial Application}

\subsection{Introduction}

This chapter presents an industrial application of one of the novel self-tuning control strategies that has been presented in this thesis. The purpose of the trial was to test a newly developed adaptive schemes on an industrial process rather than to design a controller for a particular loop. The process is typical of many in the chemical process industries in that it exhibits a long and varying time delay. The results demonstrate the applicability of this methodology in an industrial environment where increasing productivity while minimizing operational cost is an important factor. The deterministic explicit self-tuning scheme based on the complete in $\mathbf{L}_{2}[0, \infty)$ orthonormal set of Laguerre functions (described in detail in Chapter 3) has been applied to the control of the exit $p H$ of the first caustic extraction stage in a bleach plant of wood pulp at a kraft mill site. The plant is modelled by an orthonormal Laguerre network put in state-space form. A simple predictive control law is proposed. Accurate assumptions about the true values of the plant order and time-delay are not needed. The new approach proved to be well suited to an industrial application because it is easy to use, it can handle time-delayed and non-minimum-phase plants, it is robust and it has been shown to be superior to any PID control that was previously used by the mill's personnel, (see, Zervos and Dumont, 1988c). 
6.2 Laguerre-based adaptive control of $\mathrm{pH}$ in an industrial bleach plant extraction stage

The main objectives of bleaching are, to remove the coloring materials still present in the fibers, to increase the brightness of the pulp, and to produce a white pulp of satisfactory physical and chemical properties to make it suitable for the manufacture of tissue grade papers. Modern kraft pulp bleaching is achieved in a multi-stage plant, using expensive chemicals such as chlorine, chlorine dioxide, caustic soda and oxygen. A typical North American bleach plant consists of chlorination (C), alkaline extraction (E), chlorine dioxide bleaching (D), alkaline extraction (E) and chlorine dioxide bleaching (D) in that sequence (CEDED) for the production of high quality pulp at low cost. Oiher sequences less often used include, CEHDED or OCEDED, (H stands for Hypo and $O$ for Oxygen).

The sequence CEDED is capable of producing pulps of very high brightness at significantly lower capital costs and is the most preferable sequence practiced in North American mills (Singh, 1979). The first alkaline extraction stage (E) of a chlorinated (C bleached) kraft pulp is of great chemical and economical importance (Axegard, 1979 ) and is normally the 2nd stage in the commercial multi-stage pulp bleaching. process. It substantially completes the process of the pulp purification which begins in the chlorination $(\mathrm{C})$ stage. Together with other techniques, it has made possible the manufacture of stronger sulfite and sulfate pulp and paper of higher brightness. It has played a significant role in improving the pulp cleanliness and it has paid for itself by reducing the bleach demand and by producing a stable brightness in the finished pulp. It is not a bleaching stage in itself but is a continuation of the delignigication in the preceding stage. It accounts for one half or more of the CE lignification.

When unbleached woodpulp arrives at the bleach plant, it still contains enough 
amounts of lignin and other chromophores and encrustants: In order to remove as much of the residual lignin as feasible without damaging the pulp, the pulp is normally first chlorinated. To improve the pulp quality, a caustic extraction stage follows to minimize expenditures for more costly oxidizing chemicals by removing the alkaline soluble portion of the lignin from the woodpulp. The caustic soda reacts with chlorinated lignin, as well as with the hypochloric acid formed during chlorination. Finally, a small amount binds to the cellulose itself. Three steps are generally required following the washing of the chlorinated pulp: (a) mixing of caustic solution (sodium hydroxide) with the pulp,$(b)$ heating to the desired temperature, and $(c)$ retention to complete the reaction. The reaction time is usually from 50 to 100 minutes depending on the grade of the pulp to be bleached. One of the most important variables that affects the pulp quality is the $p H$ at the end of that stage, i.e. at the exit of the first caustic extraction tower. The $\mathrm{pH}$ of the feed stream is around 2 , while the target $\mathrm{pH}$ after the tower is usually between 10 and 10.5. It is of great importance both chemical and economical to keep its value as more constant as possible and close to specifications (in the range $10-12)$. Low $p H$ values degrade the pulp quality where large values prove to be of no substantial benefit since the cost of the additional consumed caustic is very high. Thus, one must operate this plant at minimal caustic consumption while maintaining pulp quality. A good control scheme will allow keeping the pulp $p H$ in acceptable good levels while minimizing the consumption of sodium hydroxide. Lab tests indicate that the titration curve of the reaction displays the characteristics of a strong acid, strong base reaction (see Figure 6.42). Around the $10.2-10.3 \mathrm{pH}$ set point significant gain changes make the control of the $\mathrm{pH}$ rather challenging. This difficulty is compounded by the fact that the buffering effect provided by the aqueous system may vary and by the time-varying nature of the dynamics. In particular, because of the propensity of 


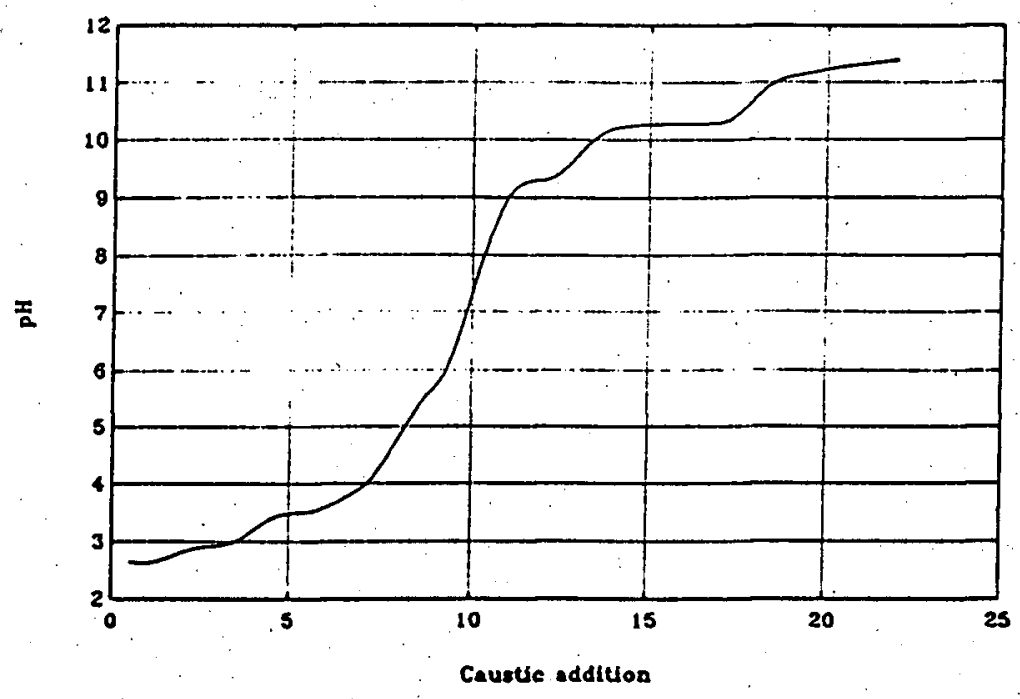

Figure 6.42: Titration curve of the caustic extraction.

the flowdown tower for channelling, the dead time in the process can be highly variable. Because, the Laguerre self-tuner is robust and efficient in presence of unknown and varying dead-times, we see this loop as a good industrial benchmark. Our main purpose is to test the Laguerre self-tuner, and compare it to the existing scheme in similar circumstances. Ideally, for this particular problem, one may want to combine this scheme with the adaptive chemical invariant technique of Waller and Gustafsson (1982), although our current knowledge of the reaction chemistry may prevent this.

Figure 6.43 shows the process and instrumentation diagram of the first alkaline extraction stage of a mill's bleaching process. The industrial control scheme uses 2 PID controllers in cascade loop mode, as shown on Figure 6.44. Only the Proportional and Integral part of the PID controller are used by the mill's personnel. while the Derivative term is zero. This cascade configuration is justified by the long retention time in the tower. The tuning of the internal pneumatic PI controller is relatively easy since it does not involve any long dead time. The tuning of the outer digital PI presents 


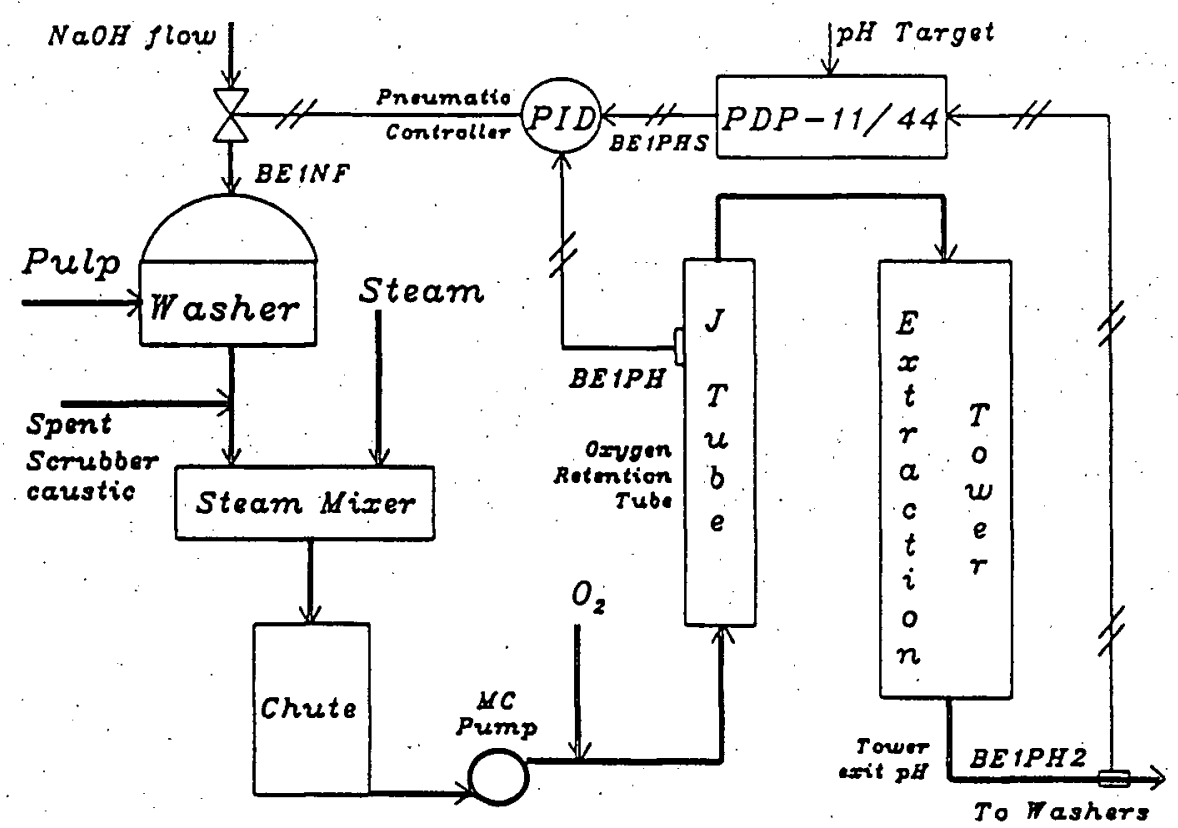

Figure 6.43: Bleach Plant. $1^{\text {st }}$ Alkaline Extraction stage.

serious problems due to the long retention time involved in the first caustic tower (40 to 70 minutes) and the inherent strong non-linearity of the loop. Figure 6.45 shows an open-loop step response of the outer loop (i.e. when the outer PI is removed and the loop is opened). The dead time is in the range of 40 to 50 minutes and the settling time 50 to 70 minutes. The dead time is thus dominant and varies as a function of the production rate and because of the propencity for channelling in the tower. Because of this, this outer loop is in manual mode more often than not. Figure 6.46 shows the tower exit $p H$ under PI computer control, when the latter is performing at its best. Note the existence of an offet, as the setpoint is 10.2 .

The choice of an adaptive controller over a fixed controller for this loop is justified by the poor knowledge of the time-varying nature of the dynamics and especially of the dead time. This latter however, seriously complicates the design of an adaptive control scheme, using standard techniques. With respect to the model order, if an ARMAX model was to be used then increasing its order means change in all parameters and as a 


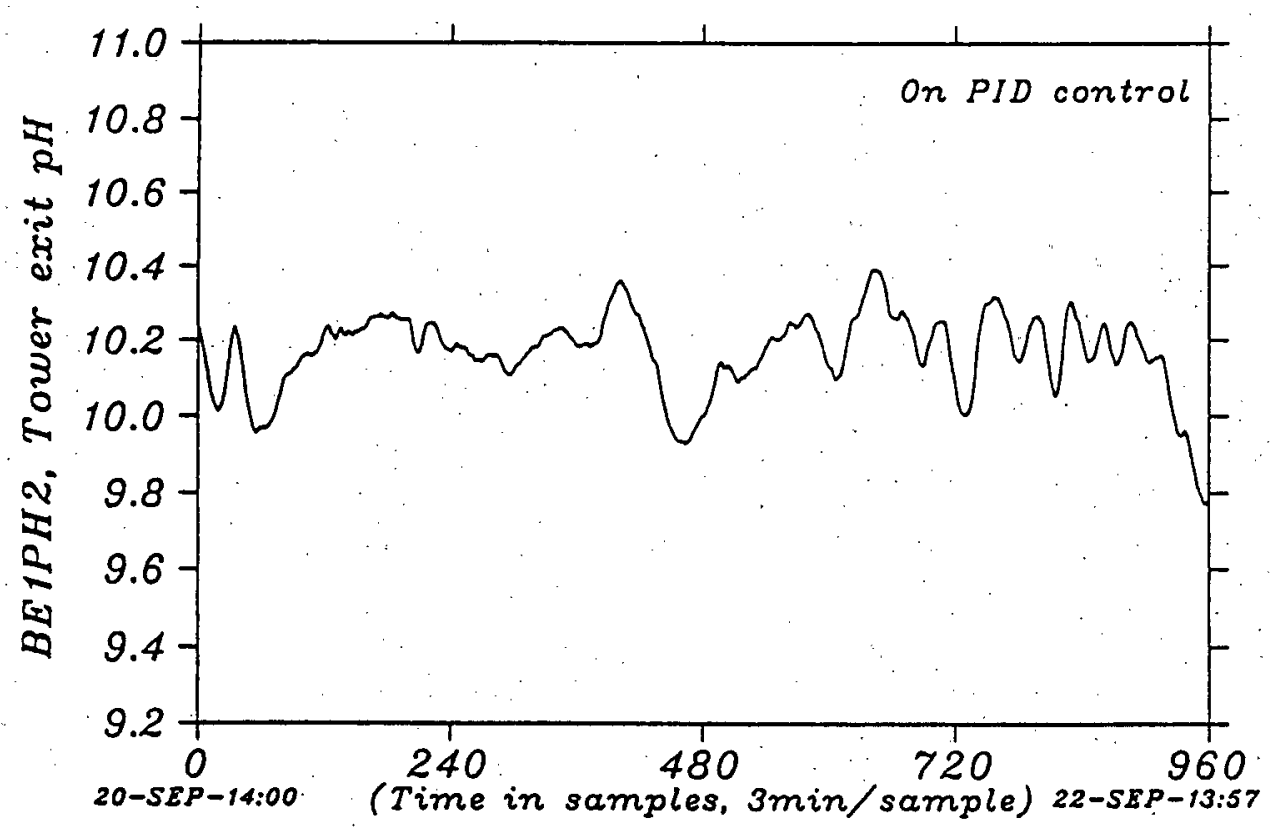

Figure 6.46: Tower exit $p H$ with the outer loop always under PID control. 3 $\mathrm{min} /$ sample-point.

result a significant transient. This is why we choose an adaptive controller based on a Laguerre series representation of the plant dynamics. The time delay is then implicitly identified as part of the Laguerre model and is not guessed as an extra parameter. Any required increase on the model order on-the-fly will not theoretically affect the low order Laguerre gains during the identification.

The algorithm was implemented in the Canadian Forest Products Ltd. Howe Sound pulp division kraft mill that produces about 670 tpd of market pulp. For the implementation of the adaptive controller, the outer PI was removed and the new self-tuner was applied in its place. The inner PI loop was left intact since the inner PI was an integral part of the control valve that manipulates the caustic flow and it never presented any problems. The algorithm was implemented in Fortran IV on the mill process control computer, a PDP-11/44 running under RSX-11M. The following parameters were chosen: sampling time $T=8 \mathrm{~min}$, number of filters $N=15$, Laguerre 
pole $p=2.0$, prediction horizon $d=9$, driver block pole $\alpha=0.5$. To initialize the parameter estimate vector $\underline{c}$, we simulated a process with a response similar to that of Figure 6.45 and used the corresponding Laguerre gains. Figure 6.47 compares, on the same graph, the performance of the new self-tuning scheme to the previously used one. The target exit $p H$ (BE1PH2) was 10.3. The graph shows a total of 2000 points, each point representing $3 \mathrm{~min}$, from September 2, 04:18 a.m, to September 6, 08:15 a.m. The new self-tuner in its final form was applied on September $4,11: 32$ a.m. $\left(1106^{\text {th }}\right.$ point on the graph). The initial transient period of the Laguerre self-tuner, clearly shown on the graph, indicates the adaptation period to the dynamics of the plant. The period that follows is indicative of the good regulation performance of the self-tuner. The time period between the $684^{\text {th }}$ and $792^{\text {th }}$ point on the graph (i.e. September 3, 14:30 - 16:18) was a short experimental test of the new self-tuning algorithm based on zero initial parameter estimates. Figure 6.48 shows a step response of the identified model using the identified Laguerre gains as recorded at September 4, 20:45 p.m. The 15 gains are given in Table 6.3. Figure 6.49 shows the output $u$ of the self-tuning controller (BE1PHS),

\begin{tabular}{||c|c||c|c||c|c||}
\hline \multicolumn{5}{||c||}{ Laguerre Gains } \\
\hline 1 & $-3.822 \mathrm{E}-04$ & 6 & -0.181398 & 11 & -0.14379590 \\
\hline 2 & $1.4819 \mathrm{E}-03$ & 7 & -1.167197 & 12 & $-9.2454 \mathrm{E}-02$ \\
\hline 3 & $7.8450 \mathrm{E}-04$ & 8 & -2.271191 & 13 & $-2.1657 \mathrm{E}-02$ \\
\hline 4 & $1.1495 \mathrm{E}-02$ & 9 & -0.939344 & 14 & $-2.1010 \mathrm{E}-02$ \\
\hline 5 & $2.1095 \mathrm{E}-02$ & 10 & -0.640596 & 15 & $5.83940 \mathrm{E}-03$ \\
\hline
\end{tabular}

Table 6.3: The 15 Laguerre gains as recorded at September 4, 20:45.

i.e. the setpoint for the inner loop. Some of the flat leveled portions of this variable in the first half of this plot represent manual control by the bleach plant operators in their effort to control efficiently the exit $p H$ by combining both computer and manual control. Figure 6.50 shows the caustic $(\mathrm{NaOH})$ flow $(\mathrm{BE} 1 \mathrm{NF})$ as it was manipulated 
by the inner PI controller. Figure 6.51 shows the $p H$ obtained from a $\mathrm{pH}$-probe inside and near the entrance of the J-Tube (BE1PH). The results clearly show the superior performance of the new self-tuning scheme and its excellent regulation capabilities despite disturbances caused by the first chlorination stage, by pulp stock flow, and by the additive spent scrubber caustic (mainly sodium hypochlorite $(\mathrm{NaOCl})$ and $\mathrm{NaOH}$ ) and oxygen flows. The long-term performance of the new self-tuning scheme was also tested by leaving the loop under adaptive control for a period of over 6 months. Figure 6.52 presents a typical 2-day run (960 points) for the period 28-30 November, 28 1987. Figure 6.53 compares the autocorrelations obtained for the $p H$ output between the loop under manual/PID control (0-1105 points on Figure 6.47) and the loop under self-tuning control (Figure 6.52). A disandvantage of the PID controller is that in order to stay at a good level of performance, it has to be retuned periodically. In addition, the $\mathrm{pH}$ probes have to periodically cleaned or replaced, thus resulting in changes in process gains requiring retuning of the PID controller. A significant benefit of the adaptive controller for the mill, is that probes can be replaced and cleaned with no requirement for retuning, as the adaptive controller will automatically adjust its parameters to the new conditions.

A major advantage of the Laguerre self-tuner is that it is easy to use and requires very little a-priori knowledge. Indeed, once the software was ported to the mill process computer, the scheme was commisioned in less than two working days. The purpose of this work was to demonstrate the potential of the Laguerre self-tuner in an industrial setting, not to deliver a new $\mathrm{pH}$ control loop to the mill. However, due to these successful tests the algorithm has been left in operation. As the current software was not written for continuous industrial operation, it has to be modified slightly. In particular, the usual jacketting for long-term operation has to be included. Also, the operators need training with the new system. Indeed, some of them not used to see 


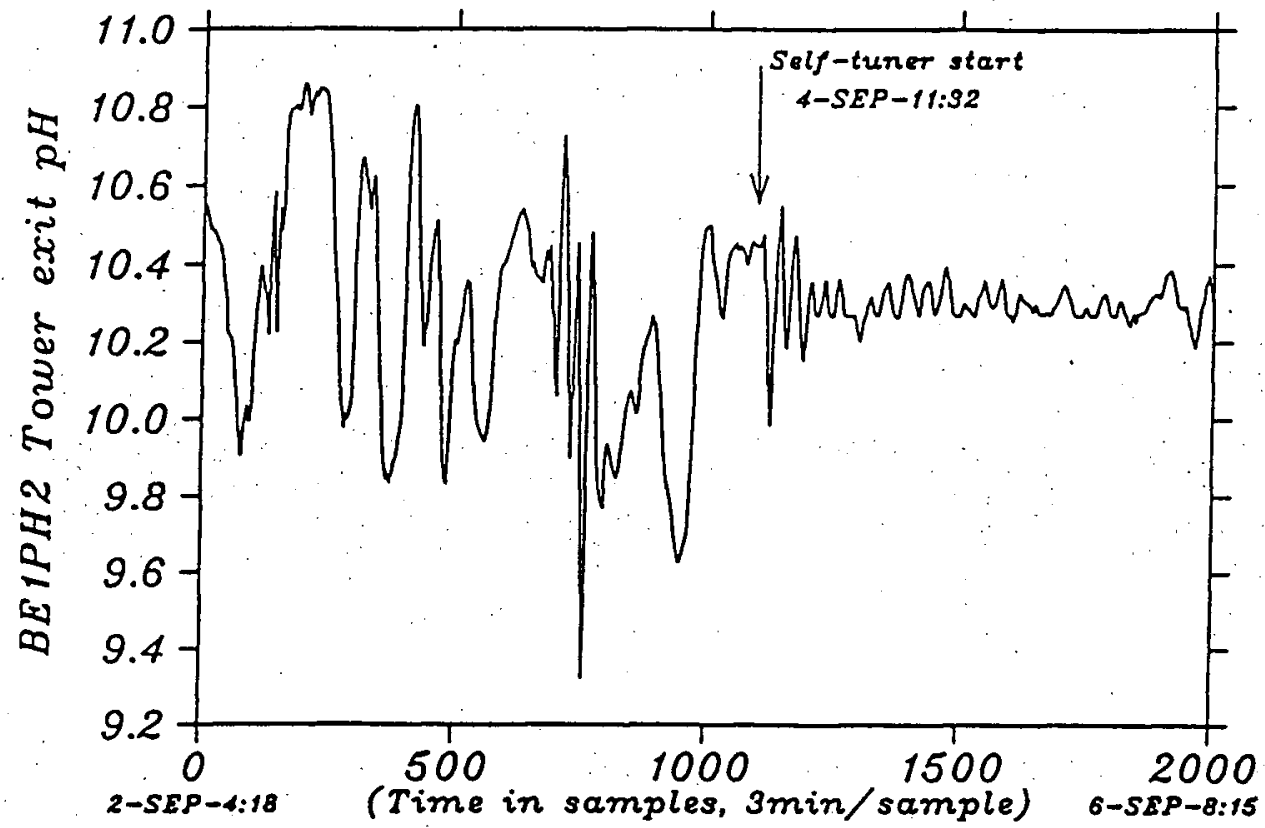

Figure 6.47: $p H$ loop. Comparison of regulation performances. $3 \mathrm{~min} / \mathrm{sample-point.}$

an active controller switch to manual control when the feel the controller is taking too much control action. A generic version of the algorithm would be very useful for use as a general-purpose controller for difficult loops in other parts of the mill. 


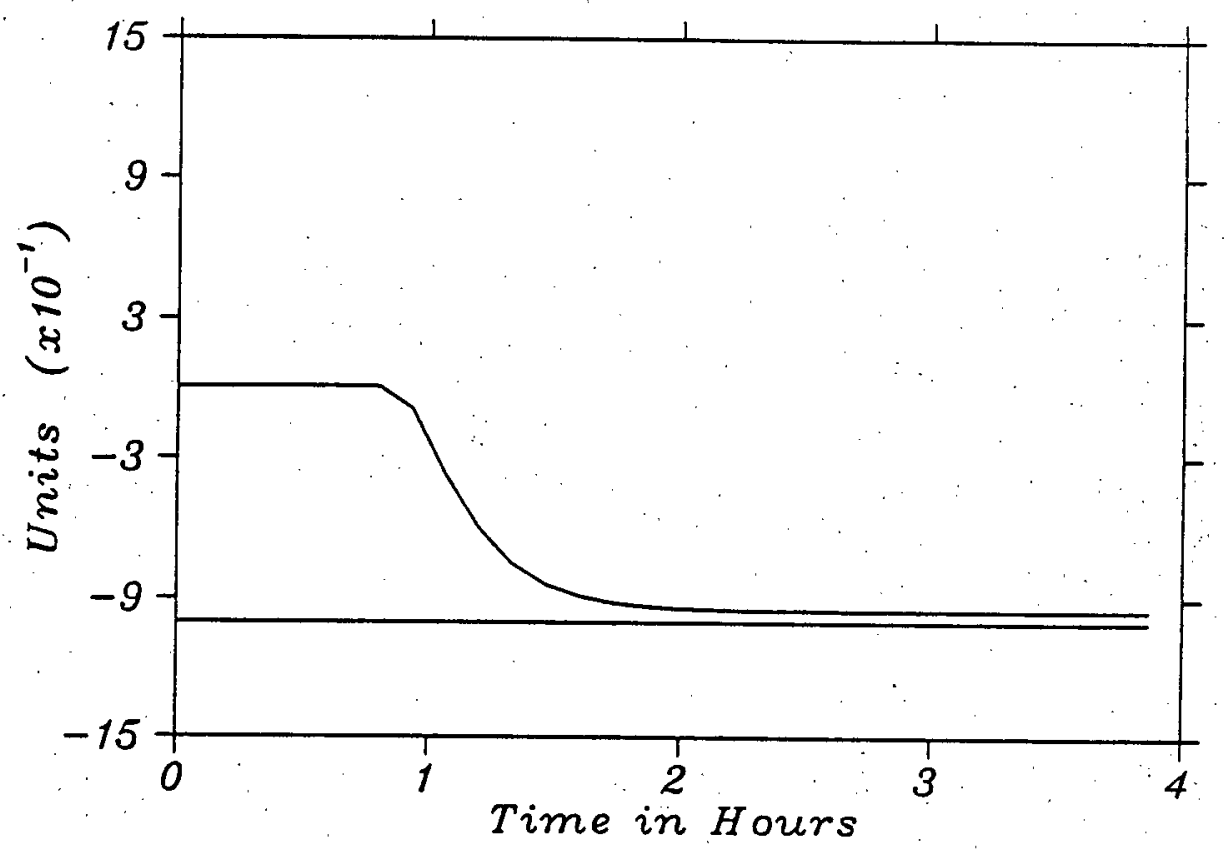

Figure 6.48: Step response of identified $p H$-loop. $8 \mathrm{~min} /$ sample-point.

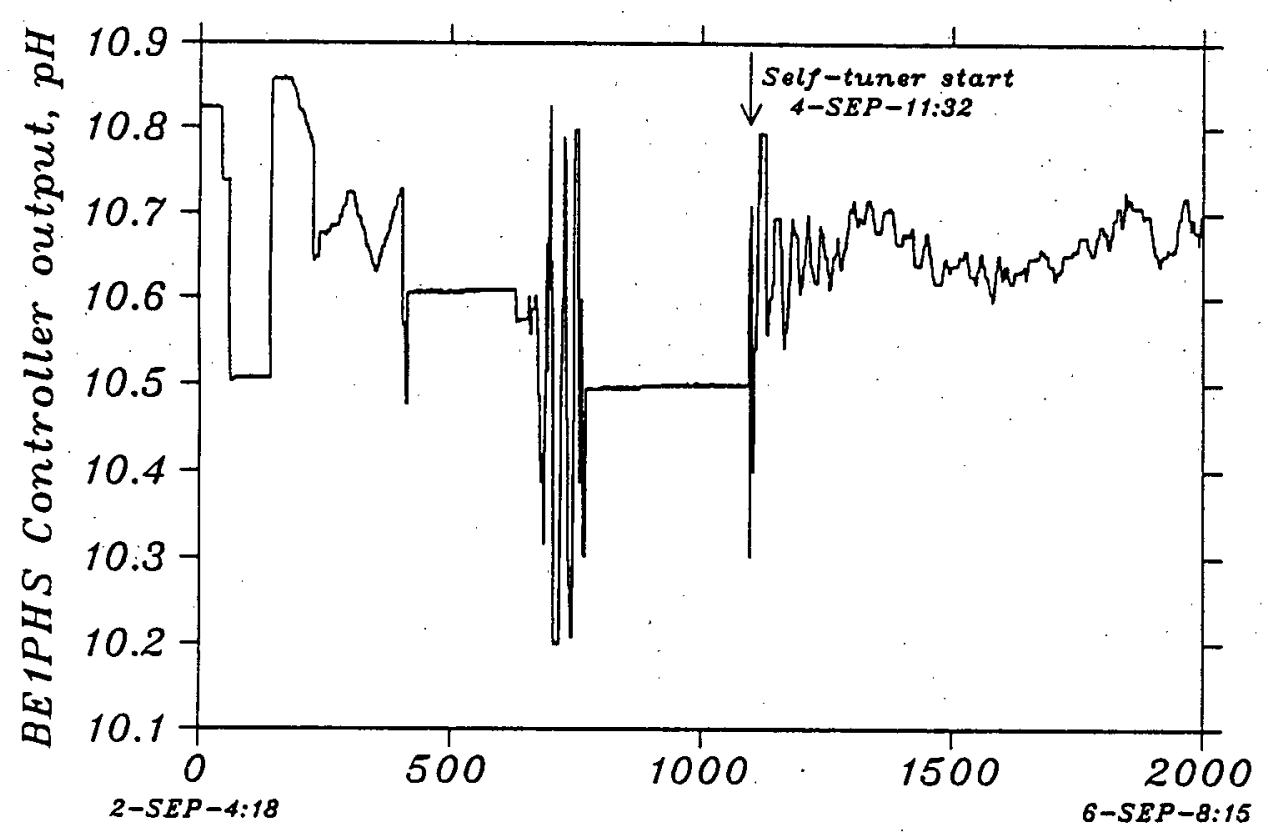

Figure 6.49: Self-tuner output in $p H$ values. $3 \mathrm{~min} /$ sample-point. 


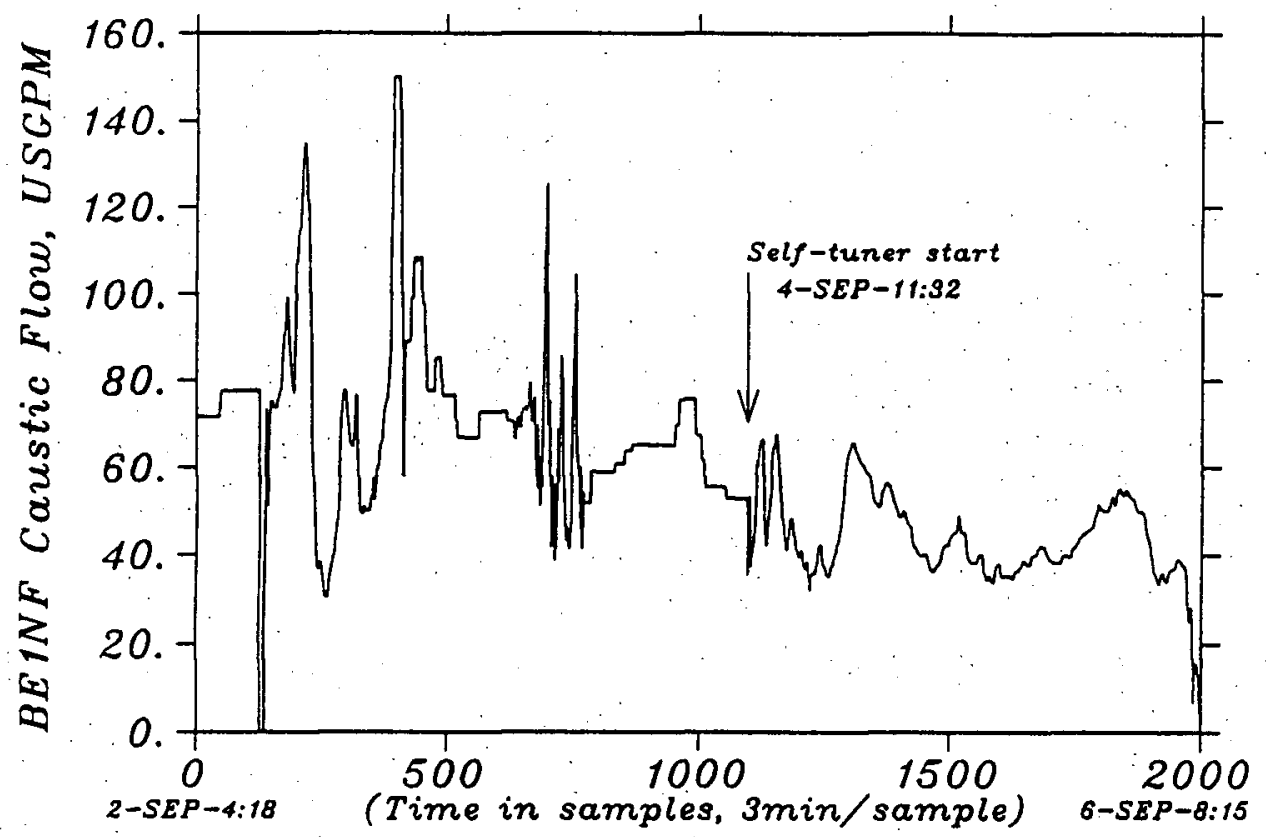

Figure 6.50: Alkaline extraction stage. Caustic flow. $3 \mathrm{~min} / \mathrm{sample-point.}$

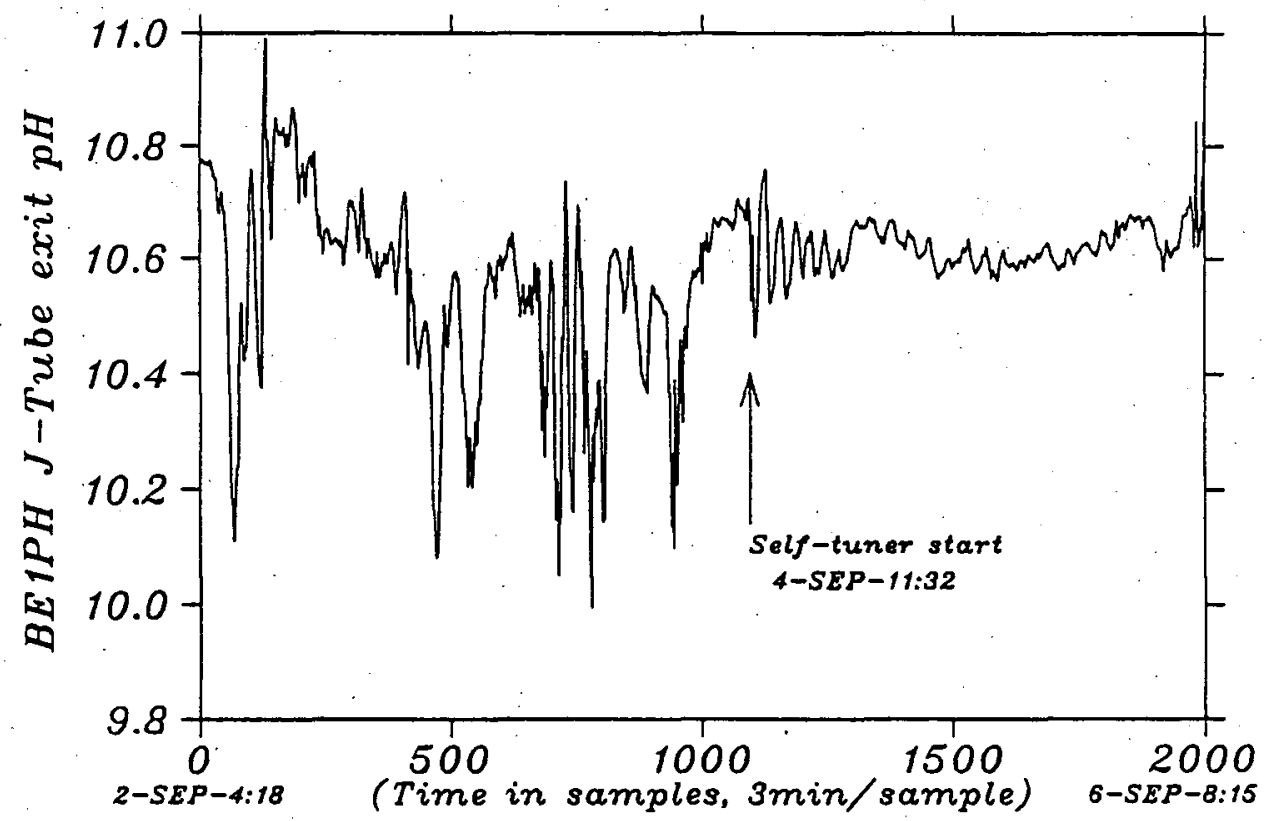

Figure 6.51: Alkaline extraction stage. J-Tube exit $p H .3 \mathrm{~min} / \mathrm{sample-point.}$ 
Chapter 6. An industrial application
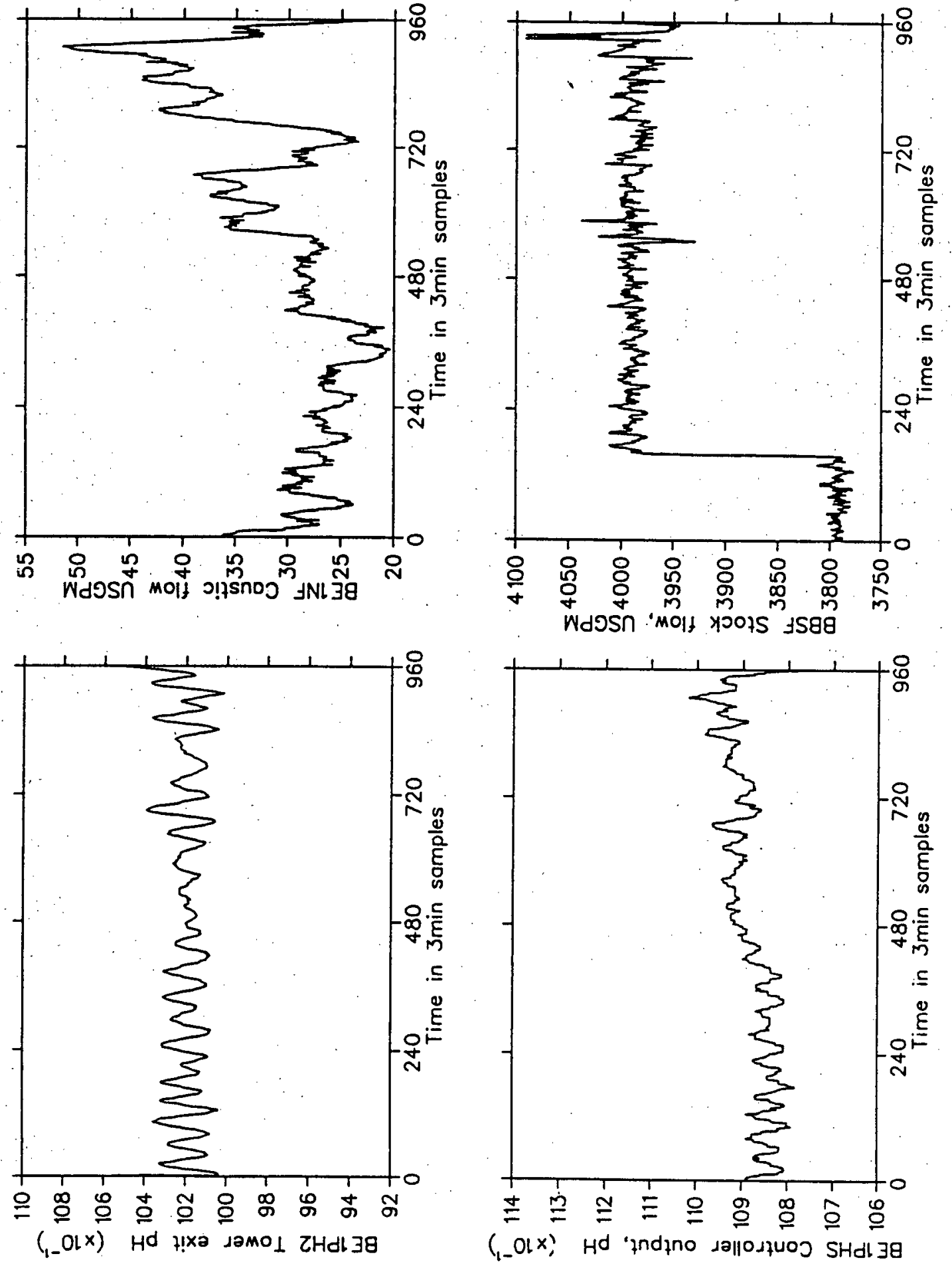

Figure 6.52: Alkaline extraction stage. Performance over the period 28-30 November, 1987, (3 $\mathrm{min} /$ sample-point). 


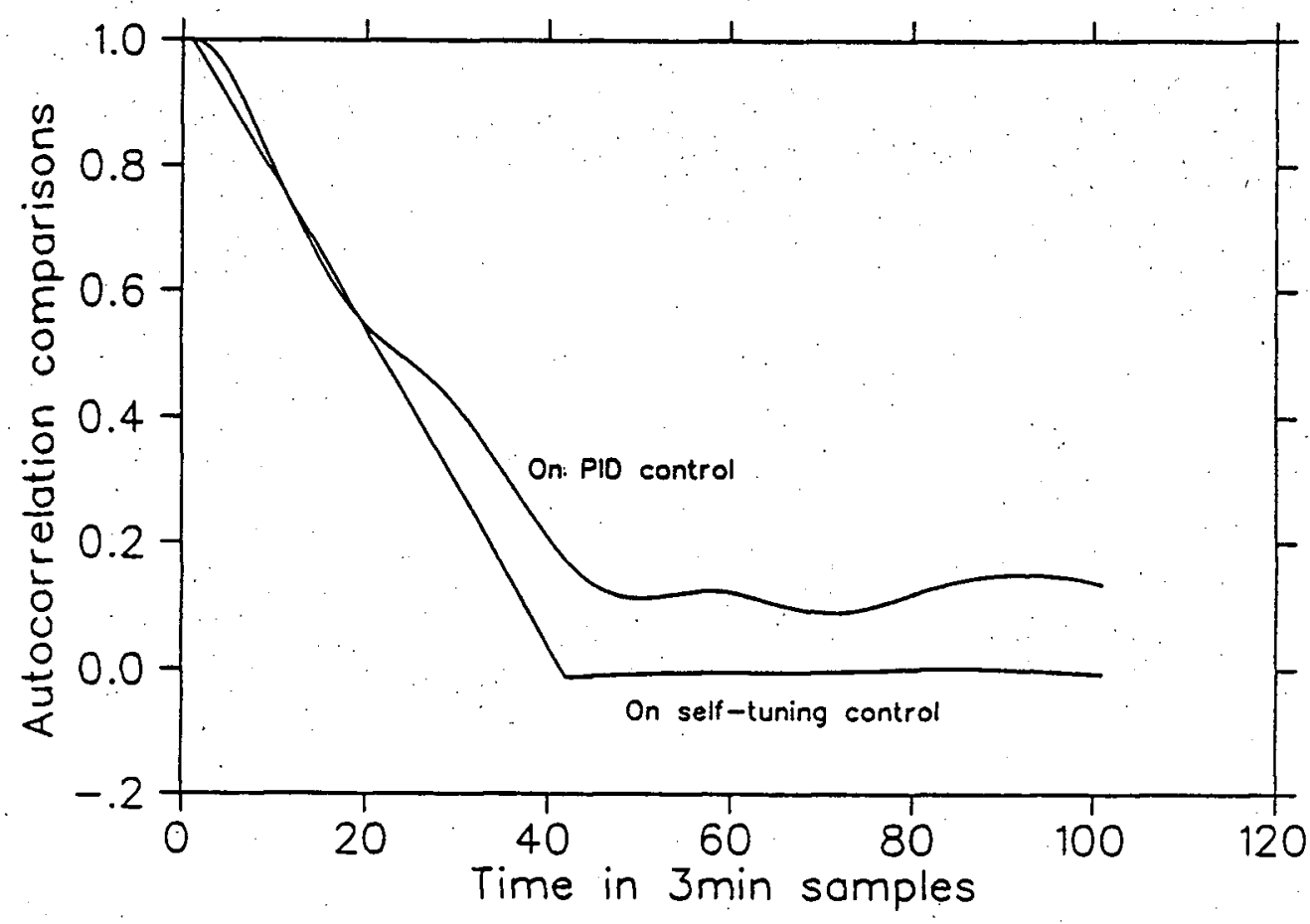

Figure 6.53: Autocorrelation comparisons between the PID/Manual and Self-tuner performance on the $p H$ loop. 


\section{Chapter 7}

\section{Using other orthonormal sets}

\subsection{A modified Laguerre set}

So far the approach to solving the adaptive self-tuning control problem using orthonormal functions has dealt mainly with Laguerre functions. Laguerre functions proved to be quite suitable for the identification of linear dynamical systems and for the development of self-tuning control strategies. However, the method is not at all restrictive and other orthonormal functions can be used. Laguerre proved to be a good candidate for implementing self-tuning control strategies because of their efficient way of handling time delays and transient signals. As an extension to this well-proven orthonormal set it is important to study the location of the poles of those functions in the frequency domain. By inspection; the poles of the Laguerre functions in the s-domain are all real and lie at the point $-p$. An immediate modification would be to have the poles lie at different real locations, $-p_{1},-p_{2}, \ldots,-p_{n}$. For simplicity, these poles could be located in equally-spaced points on the negative real axis between 2 boundary points. Then the Laplace transform of the $n^{\text {th }}$ such function would be, (Kautz, 1954),

$$
F_{n}(s)=\sqrt{2 p_{n}} \frac{\left(s-p_{1}\right)\left(s-p_{2}\right) \cdots\left(s-p_{n-1}\right)}{\left(s+p_{1}\right)\left(s+p_{2}\right) \cdots\left(s+p_{n-1}\right)\left(s+p_{n}\right)}
$$

Such a set of orthonormal functions could be represented by the Ladder Network of Figure 7.54. Note that in contrast with the Laguerre Ladder Network (Figure 2.2 the all-pass phase-shift chain is now a lead-lag chain. 


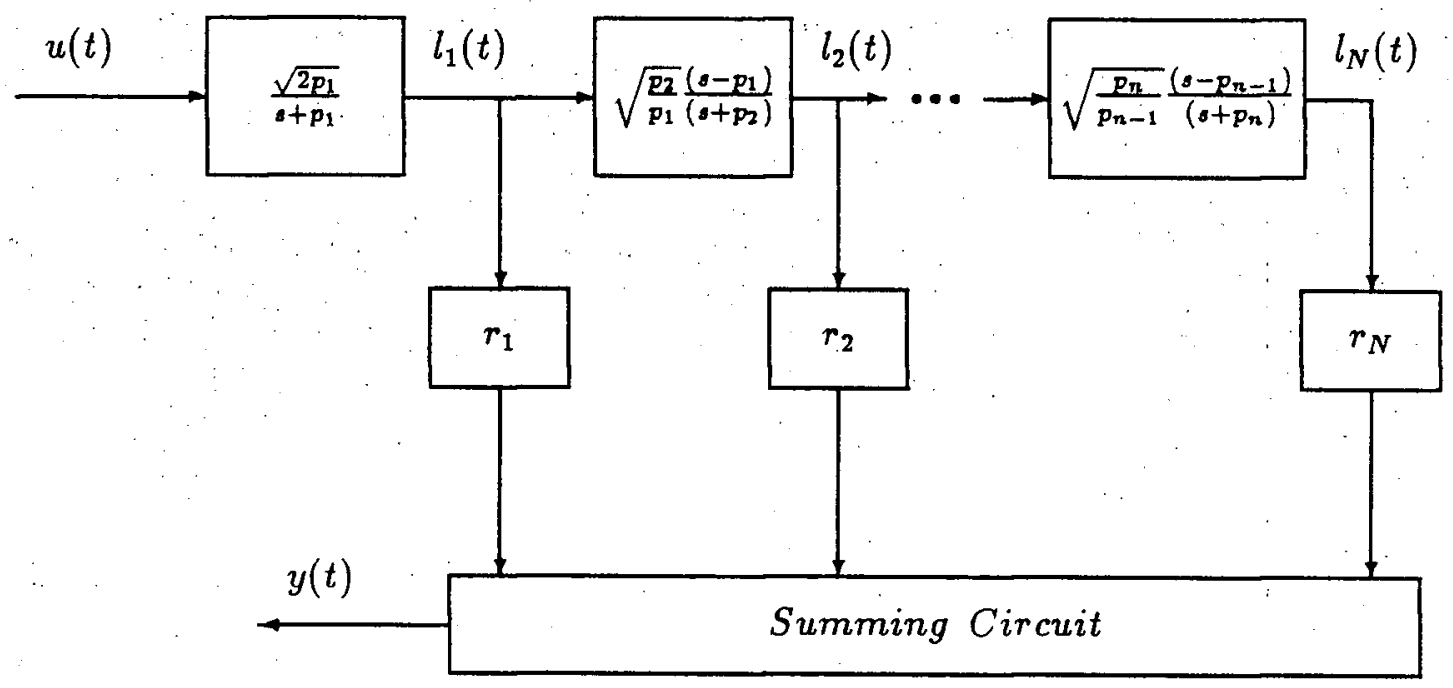

Figure 7.54: Modified Laguerre Ladder Network. 
It will be noted that the zeros of each of these functions is located at the negatives of the poles except.for the new pole not present in the previous function. Expanding each function into partial fractions and performing an inverse Laplace transform we get,

$$
f_{n}(t)=\sum_{i=1}^{n} \gamma_{i n} e^{-p_{i} t}
$$

where $\gamma_{i n}$ is the (real) residue in the pole at $-p_{i}$ of the $F_{n}(s)$.

Applying the same technique as in Sections 2.2.4 and 2.3 we can represent the orthonormal Ladder Network of Figure 7.54 in state-space form. In particular, defining the $N$-dimensional state vector $\underline{l}(t)$ as,

$$
\underline{l}^{T}(t)=\left[\begin{array}{llll}
l_{1}(t) & l_{2}(t) & \ldots & l_{N}(t)
\end{array}\right]
$$

this state-space formulation has now the form,

$$
\underline{l}(t+1)=A \underline{l}(t)+\underline{b} u(t)
$$

$A$ is again a lower triangular $N \times N$ matrix where the same elements are found respectively across the diagonal or every subdiagonal. If $T$ is the sampling period then,

$$
A=\left[\begin{array}{ccccc}
\tau_{1} & 0 & \cdots & \cdots & 0 \\
\rho_{2} \frac{\tau_{1} \mu_{2}+\nu_{2}}{T} & \tau_{2} & \cdots & \cdots & 0 \\
\vdots & \vdots & \vdots & \vdots & \vdots \\
\left(\frac{\tau_{1} \mu_{2}+\nu_{2}}{T^{N-1}} \prod_{i=2}^{N} \rho_{i} \prod_{i=3}^{N} \mu_{i}\right) & \left(\frac{\tau \mu_{3}+\nu_{3}}{T^{N-2}} \prod_{i=3}^{N} \rho_{i} \prod_{i=4}^{N} \mu_{i}\right) & \cdots & \rho_{2} \frac{\tau_{\mu_{2}+\nu_{2}}}{T} & \tau_{n}
\end{array}\right]
$$

and

$$
\underline{b}^{T}=\left[\begin{array}{llll}
\xi & (\xi / T) \rho_{2} \mu_{2} & \cdots & \left(\xi / T^{N-1}\right) \prod_{i=2}^{N} \rho_{i} \prod_{i=2}^{N} \mu_{i}
\end{array}\right]
$$

where,

$$
\tau_{i}=e^{-p_{i} T}
$$




$$
\begin{gathered}
\rho_{i}=\sqrt{\frac{\ddot{p}_{i-1}}{p_{i}}} \\
\mu_{i}=T+\left(p_{i-1}+p_{i}\right)\left(\tau_{i}-1\right) /\left(p_{i-1} p_{i}\right) \\
\nu_{i}=-T \tau_{i}-\left(p_{i-1}+p_{i}\right)\left(\tau_{i}-1\right) /\left(p_{i-1} p_{i}\right) \\
\xi=\sqrt{2 p_{1}}\left(1-\tau_{1}\right) / p_{1}
\end{gathered}
$$

The advantage of using such an orthonormal set of functions is that one could specify a range on the negative real axis for the location of the real poles $p_{i}$ instead of just a single point. This will in effect provide wider coverage on the assumption of the dominant time-constant of an unknown plant when starting the identification.

The development of output predictive expressions and self-tuning schemes, either deterministic, or stochastic, based on the above set follow exactly the same procedures outlined for the Laguerre set in Chapters 3, 4, respectively. Multivariable schemes follow exactly the procedures outlined in Chapter 5.

\subsection{A set with discrete complex exponentials}

In dealing with the problem of signal analysis, one set of very useful component functions is the orthonormal exponential functions. Previous studies have concentrated mostly, on continuous exponentials with real exponents. The work so far in this thesis emphasized mostly exponential functions with real exponents because of their simplicity. The motivation to develop a new set with complex poles is twofold. The first is to reduce the number of filters required for the identification of underdamped systems. This is particularly important for parsimonious identification of multivariable systems that exhibit underdamped oscillations in their response. Second, according to studies on signal representation (Young and Huggins, 1962), for nearly all classes of signals the exponents of an optimum least-squares representation have turned out to be complex. 
As a result, we shall devote our effort in this section toward the derivation of identification and control schemes based on discrete exponential functions with complex exponents.

The Z-transforms of continuous orthonormal exponentials are not themselves orthogonal in Z-domain. To avoid this difficulty, we choose at the outset, discrete basis functions which are orthogonal in the $z$-domain and have poles in the $z$-domain corresponding to the s-domain poles of the continuous orthonormal exponentials. The zeros are different, however, although they are chosen in such a way that as the sampling interval approaches zero, the chosen basis functions will approach the s-domain orthonormal exponentials (Young and Huggins, 1962). Given a set of exponential functions with poles at $-p_{k} \pm j q_{k},\left(p_{k}>0\right)$, a set of orthonormal exponential functions may be constructed in the frequency domain as follows,

$$
\begin{gathered}
F_{2 k-1}(s)=b_{2 k-1}^{\prime} \frac{\left(s-\gamma_{2 k-1}^{\prime}\right)}{\left(s^{2}+\alpha_{k} s+\beta_{k}\right)} \prod_{i=1}^{k-1} \frac{\left(s^{2}-\alpha_{i} s+\beta_{i}\right)}{\left(s^{2}+\alpha_{i} s+\beta_{i}\right)} \\
F_{2 k}(s)=b_{2 k}^{\prime} \frac{\left(s-\gamma_{2 k}^{\prime}\right)}{\left(s^{2}+\alpha_{k} s+\beta_{k}\right)} \prod_{i=1}^{k-1} \frac{\left(s^{2}-\alpha_{i} s+\beta_{i}\right)}{\left(s^{2}+\alpha_{i} s+\beta_{i}\right)}
\end{gathered}
$$

where,

$$
\begin{gathered}
s_{k}=-p_{k}+j q_{k}, \quad s_{k}^{*}=-p_{k}-j q_{k} \\
\alpha_{k}=2 p_{k}, \quad \beta_{k}=p_{k}^{2}+q_{k}^{2} .
\end{gathered}
$$

i.e. the zeros of the polynomial $\left(s^{2}+\alpha_{k} s+\beta_{k}\right)$ are: $-p_{k} \pm j q_{k}$. And the constants $b_{i}^{\prime}$ are the normalization constants. The zeros $\gamma_{i}^{\prime}$ can be chosen rather arbitrarily under the restriction of orthogonality,

$$
\frac{1}{2 \pi j} \int_{-j \infty}^{j \infty} F_{2 k-1}^{*} F_{2 k}(s) d s=0
$$

which in our case is equivalent to: $\gamma_{2 k-1}^{\prime} \gamma_{2 k}^{\prime}=\beta_{k}$. (Note that: "*" stands for complex conjugate). As we mentioned before orthogonality in the s-domain does not yield 
orthogonality in the $\mathrm{z}$-domain representatives of these continuous functions. Therefore, we would be more interested in deriving a discrete orthonormal set of exponential functions, with poles in the $\mathrm{z}$-domain related to the poles in the s-domain in such a way that,

$$
\begin{aligned}
& z_{k}=e^{\delta_{k} T}=e^{\left(-p_{k}+j q_{k}\right) T} \\
& z_{k}^{*}=e^{\delta_{k}^{*} T}=e^{\left(-p_{k}-j q_{k}\right) T}
\end{aligned}
$$

Generally speaking, picking $N$ pairs of s-plane complex poles $\left(s_{k}, s_{k}^{*}\right)$ then we can construct a discrete set of orthonormal functions as follows,

$$
\begin{gathered}
F_{2 k-1}\left(q^{-1}\right)=b_{2 k-1} \frac{\left(1-\gamma_{2 k-1} q^{-1}\right)}{\left(1-z_{k} q^{-1}\right)\left(1-z_{k}^{*} q^{-1}\right)} \prod_{i=1}^{k-1} z_{i} z_{i}^{*} \frac{\left(z_{i}-q^{-1}\right)\left(z_{i}^{*}-q^{-1}\right)}{\left(1-z_{i} q^{-1}\right)\left(1-z_{i}^{*} q^{-1}\right)} \\
F_{2 k}\left(q^{-1}\right)=b_{2 k} \frac{\left(1-\gamma_{2 k} q^{-1}\right)}{\left(1-z_{k} q^{-1}\right)\left(1-z_{k}^{*} q^{-1}\right)} \prod_{i=1}^{k-1} z_{i} z_{i}^{*} \frac{\left(z_{i}-q^{-1}\right)\left(z_{i}^{*}-q^{-1}\right)}{\left(1-z_{i} q^{-1}\right)\left(1-z_{i}^{*} q^{-1}\right)}
\end{gathered}
$$

where the $\gamma_{i}$ satisfy the orthogonality condition,

$$
\frac{1}{2 \pi j T} \oint F_{i}^{*}\left(q^{-1}\right) F_{j}(q) d z / z=0, \quad i \neq j
$$

and the $b_{i}$ satisfy the normality condition,

$$
\frac{1}{2 \pi j T} \oint F_{i}^{*}\left(q^{-1}\right) F_{i}(q) d z / z=1
$$

The contour of integration was taken along the unit circle. In that way as the sampling interval $\mathrm{T}$ approaches zero, the discrete orthonormal exponentials approach the ordinary continuous exponential functions. From equation (7.214) we can derive the following expression for the $\gamma_{i}$,

$$
\gamma_{2 k}=\frac{\gamma_{2 k-1}\left(z_{2 k-1}+z_{2 k-1}^{*}\right)-\left(1+z_{2 k-1} z_{2 k-1}^{*}\right)}{\gamma_{2 k-1}\left(1+z_{2 k-1} z_{2 k-1}^{*}\right)-\left(z_{2 k-1}+z_{2 k-1}^{*}\right)}
$$

and from equation (7.215) we can derive the following expression for the $b_{i}$,

$$
b_{i}^{2}=T \frac{\left(1-z_{j} z_{j}^{*}\right)\left(1-z_{j}^{2}\right)\left(1-z_{j}^{* 2}\right)}{\left(1+\gamma_{i}^{2}\right)\left(1+z_{j} z_{j}^{*}\right)-2 \gamma_{i}\left(z_{j}+z_{j}^{*}\right)}
$$


where $j=$ integer quotient of $[(i+1) / 2]$. One advantage of orthonormal exponentials is the relative simplicity in constructing an orthonormal filter by cascading a number of filter sections as shown in Figure 7.55. Each filter $U_{i}\left(q^{-1}\right)$ in Figure 7.5.5 is a unitary operator and $x_{i}(t)$ is its output. The $U_{i}$ are of the form,

$$
U_{i}\left(q^{-1}\right)=\frac{\left(z_{i}-q^{-1}\right)\left(z_{i}^{*}-q^{-1}\right)}{\left(1-z_{i} q^{-1}\right)\left(1-z_{i}^{*} q^{-1}\right)}
$$

and the functions $L_{i}$ are given by the formula;

$$
L_{i}\left(q^{-1}\right)=b_{i} \frac{\left(1-\gamma_{i} q^{-1}\right)}{\left(1-z_{j} q^{-1}\right)\left(1-z_{j}^{*} q^{-1}\right)}
$$

where $j=$ integer quotient of $[(i+1) / 2]$.

The discrete zeros $\left(\gamma_{i}\right)$ and poles $\left(z_{i}\right)$ are computed from their s-plane counterparts. To simplify the choice of the (s-plane) zeros and poles a rather simple procedure is suggested where a semi-circular array of poles with equidistant real-part spacing is constructed. This requires only the radius of the LHP semi-circle. The real zeros of the odd-numbered filters have the same real part as the correspondent poles of the associated set and the zeros of the even-numbered functions are simply calculated from equation (7.216). If the number $N$ of filters is odd then the last zero is the negative real part (i.e mirror image) of the real part of corresponding complex pole. It will be recalled that a semi-circular array of poles has proved useful for the reproduction of signal irregularities and discontinuities (Kautz, 1954).

The outputs $x_{k}$ from the unitary operators in Figure 7.55 can be expressed using the formula,

$$
\begin{aligned}
x_{k}(t)= & \psi_{2 k-1,1} x_{k}(t-1)+\psi_{2 k-1,2} x_{k}(t-2) \\
& +\sum_{i=1}^{k-1}(-1)^{k-i}\left\{\psi _ { 2 i + 3 , 2 } \psi _ { 2 i + 5 , 2 } \cdots \psi _ { 2 k - 1 , 2 } \left[\left(\psi_{2 i+1,2} \psi_{2 i-1,1}+\psi_{2 i+1,1}\right) x_{i}(t-1)\right.\right. \\
& \left.\left.-\left(1-\psi_{2 i+1,2} \psi_{2 i-1,2}\right) x_{i}(t-2)\right]\right\} \\
& +(-1)^{k} \psi_{2 k-1,2} \cdots \psi_{3,2}\left[\psi_{1,2} u(t)+\psi_{1,1} u(t-1)-u(t-2)\right]
\end{aligned}
$$




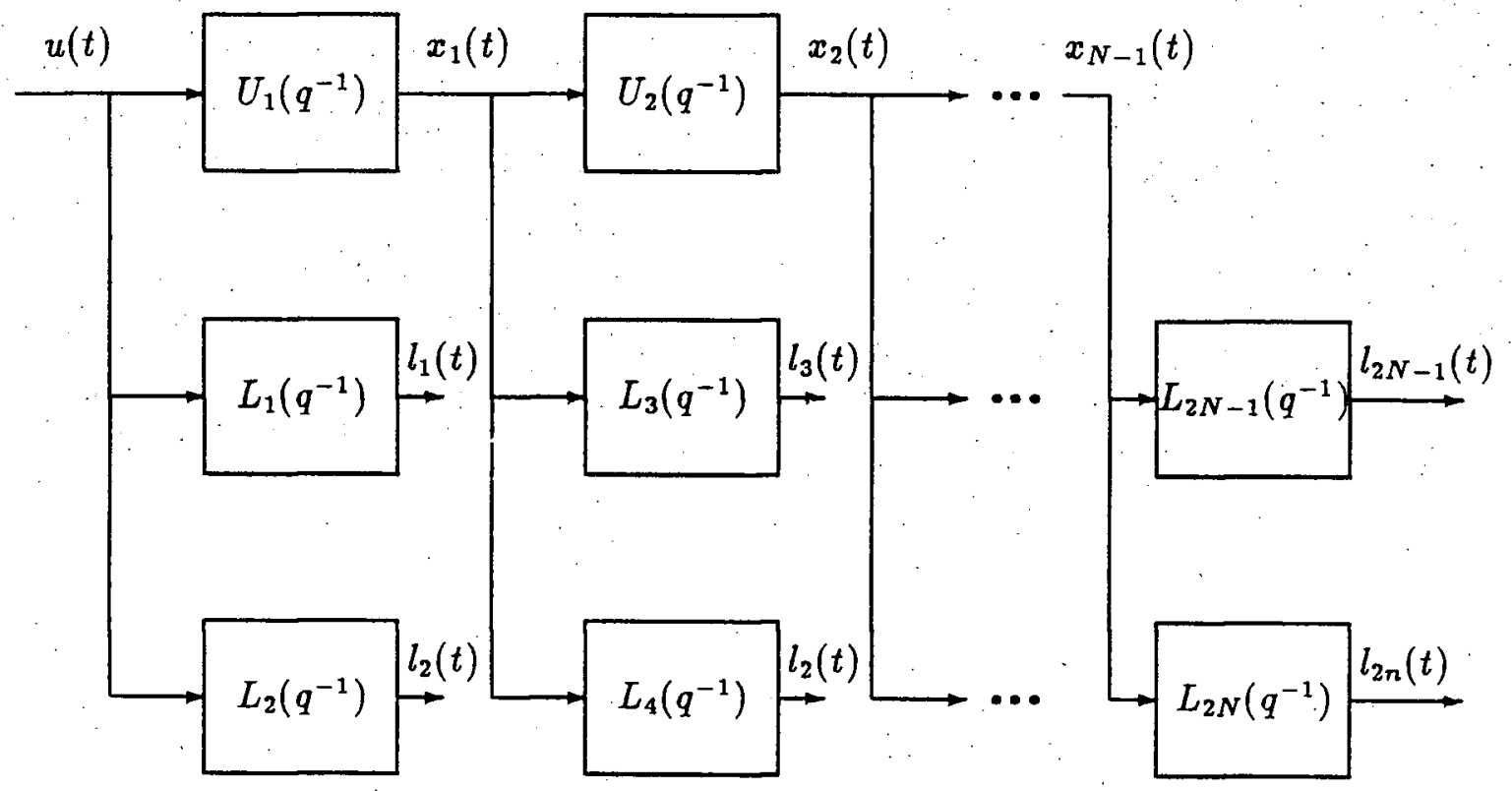

Figure 7.55: Ladder Network for the orthonormal set of functions with complex poles. 
where,

$$
\psi_{i, 1}=z_{j}+z_{j}^{*} \quad, \quad \dot{\psi}_{i, 2}=-z_{j} z_{j}^{*}
$$

where $j=$ integer quotient of $[(i+1) / 2]$. Predictive expressions can now be derived for the outputs $l_{i}$ (see Figure 7.55) based on predictive expressions for $x_{i}$. We can derive (in the same way as it was described in Chapter 3) the general formula fot the $d$-step ahead predictive expression of the outputs $l_{i}$. As an example the predictive expressions for the first two functions $(i=1,2)$ are,

$$
\begin{aligned}
l_{i}(t+d)= & {\left[\psi_{i 1}^{d}+\frac{(d-1)}{1 !} \psi_{i 1}^{d-2} \psi_{i 2}+\frac{(d-2)(d-3)}{2 !} \psi_{i 1}^{d-4} \psi_{i 2}^{2}\right.} \\
& +\frac{(d-3)(d-4)(d-5)}{3 !} \psi_{i 1}^{d-6} \psi_{i 2}^{3} \\
& \left.+\frac{(d-4)(d-5)(d-6)(d-7)}{4 !} \psi_{i 1}^{d-8} \psi_{i 2}^{4}+\cdots\right] l_{i}(t) \\
& +\left[\psi_{i 1}^{d-1} \psi_{i 2}+\frac{(d-2)}{1 !} \psi_{i 1}^{d-3} \psi_{i 2}^{2}+\frac{(d-3)(d-4)}{2 !} \psi_{i 1}^{d-5} \psi_{i 2}^{3}\right. \\
& \left.+\frac{(d-4)(d-5)(d-6)}{3 !} \psi_{i 1}^{d-7} \psi_{i 2}^{4}+\cdots\right] l_{i}(t-1) \\
& +b_{i}\left[\left[\psi_{i 1}^{d-1}+\frac{(d-2)}{1 !} \psi_{i 1}^{d-3} \psi_{i 2}+\frac{(d-3)(d-4)}{2 !} \psi_{i 1}^{d-5} \psi_{i 2}^{2}\right.\right. \\
& \left.+\frac{(d-4)(d-5)(d-6)}{3 !} \psi_{i 1}^{d-7} \psi_{i 2}^{3} \cdots\right](u(t)-u(t-1))
\end{aligned}
$$

A predictive expression for the output $y(t)$ can then be derived based on the predictive expressions of the functions $l_{i},(i=1, \ldots, N)$. In general terms this could be expressed as,

$$
y(t+d)=\mathcal{Y}\left(l_{1}(t), \ldots, l_{N}(t), l_{1}(t-1), \ldots, l_{N}(t-1), u(t), u(t-1)\right)
$$

Defining a reference trajectory in the same way as we did in Chapter 3 and equating it to the function in the r.h.s. of the above equation then the required control input can be computed in order to complete the self-tuning closed-loop scheme,

$$
u(t)=\mathcal{U}\left(y_{r}(t+d), y(t), u(t), u(t-1), l_{1}(t), \ldots, l_{N}(t), l_{1}(t-1), \ldots, l_{N}(t-1)\right)
$$


The example that follows shows simulation results that illustrate the use of this orthonormal set with complex poles for identification purposes.

Example 7.1: Consider the $2^{\text {nd }}$ order underdamped non-minimum phase plant of the form,

$$
G\left(q^{-1}\right)=\frac{\left(1+1.5 q^{-1}\right)}{\left(1-q^{-1}+0.72 q^{-2}\right)} q^{-1}
$$

Measurement noise of $\sigma=0.1$ was added to the output and then an open-loop identification experiment was performed by injecting 3 PRBS of 64 points to the input. The purpose of the identification was to evaluate the set with complex exponentials and compare it with the regular Laguerre set. Both sets performed well, and as expected the complex set used less filters ( 6 instead of 12 ) to achieve the same identification accuracy. This is advantageous especially when the plant's response is oscillatory or when implementing multivariable schemes of underdamped systems. Figure 7.56 $(N=12, p=0.7, \alpha=0.7)$ and Figure $7.57(N=6$, Radius $=0.9, \alpha=0.7)$ show the step responses in both cases and compare them with the plant's true one. Figure 7.58 shows the comparison between the obtained Bode plots of the true plant and the identified one when using the set of complex exponentials.

It is worth-while to mention here that the complex set performed also well on identifying plants with overdamped responses and on plants exhibiting rather long time delays. 


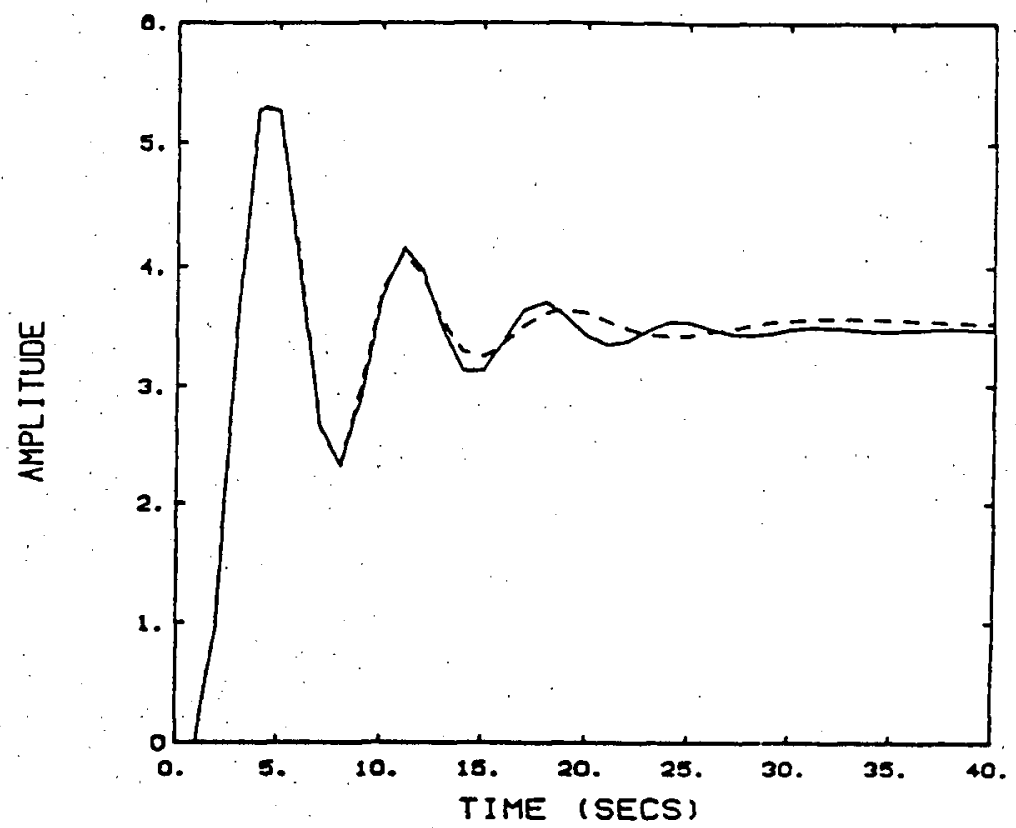

Figure 7.56: Example 7.1: Step-response comparison of the true plant (solid) and the identified one (dash line) using the Laguerre set $(N=12)$.

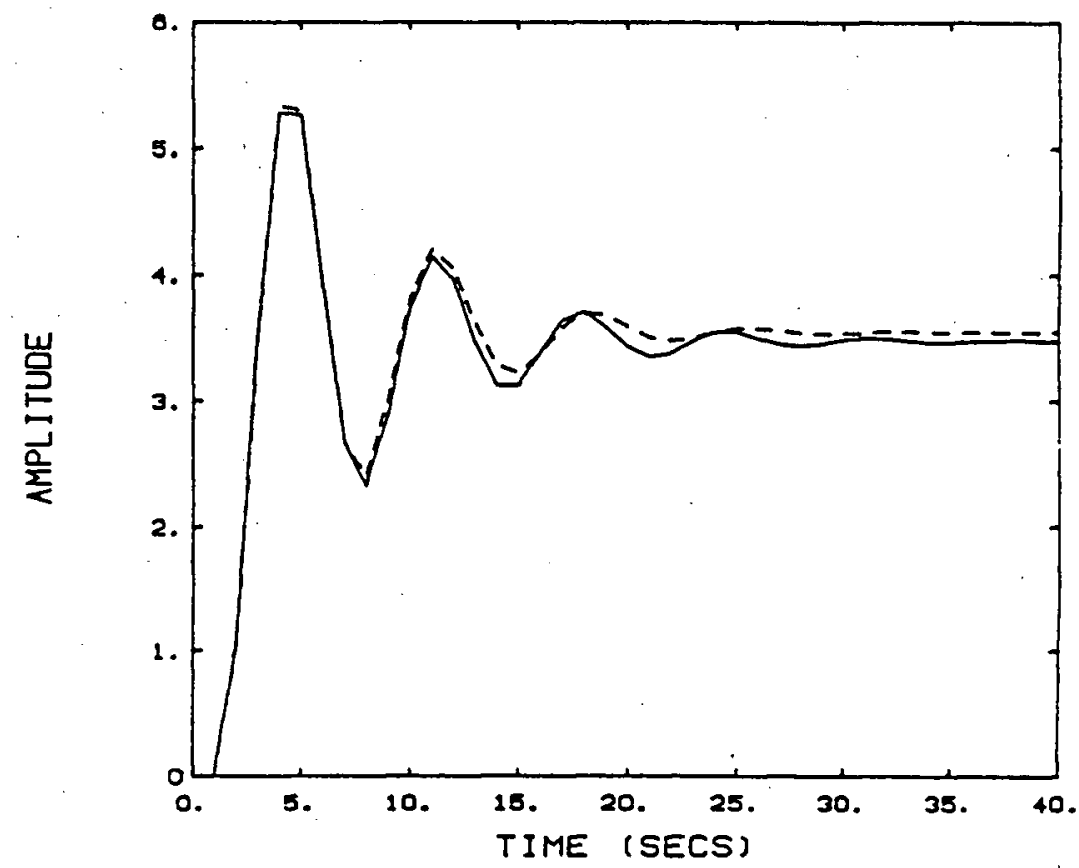

Figure 7.57: Example 7.1: Step-response comparison of the true plant (solid) and the identified one (dash line) using the Complex set $(N=6)$. 

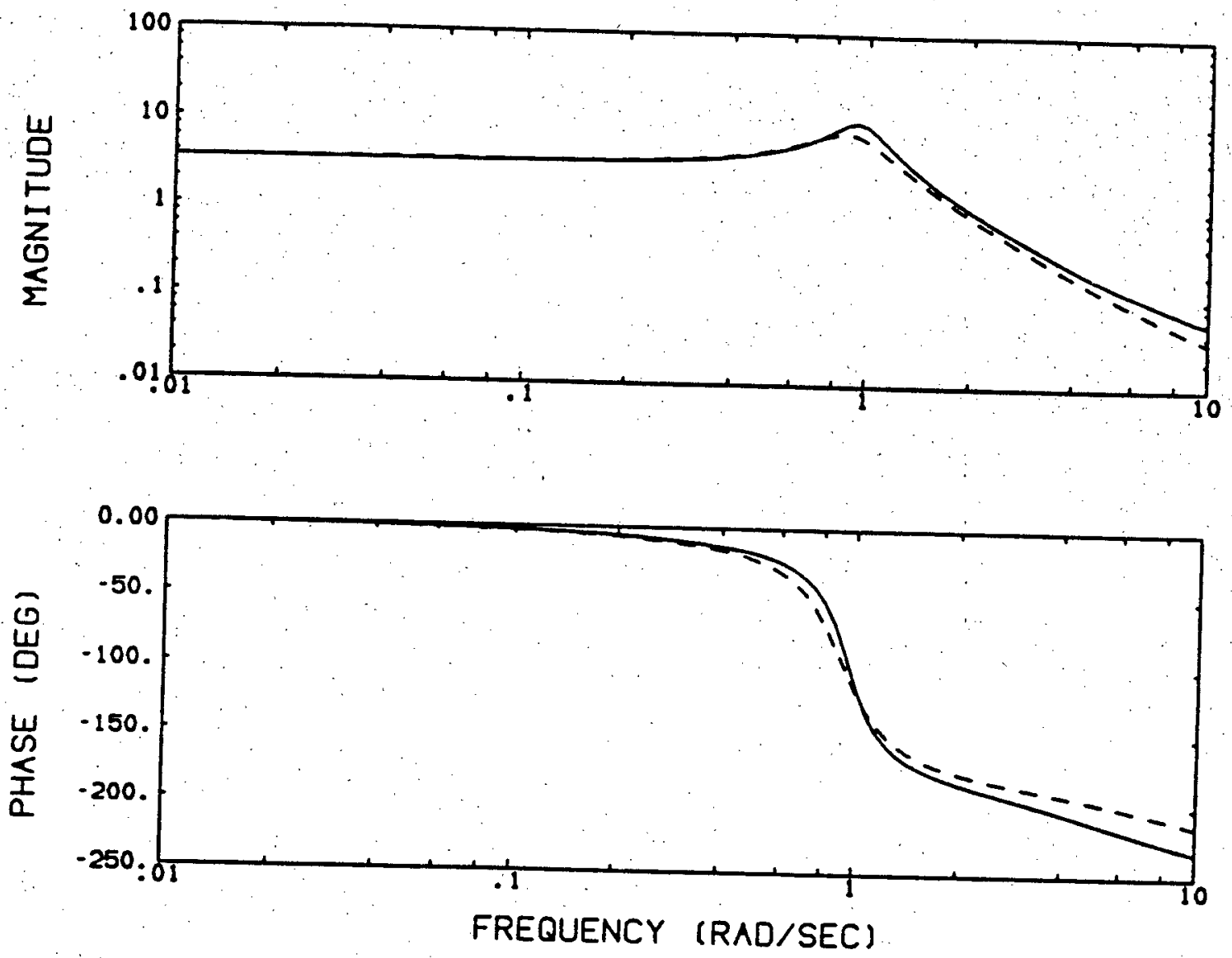

Figure 7.58: Example 7.1: Bode-plot comparison of the true plant (solid) and the identified one (dash line) using the Complex set $(N=6)$. 


\section{Chapter 8}

\section{Conclusions}

In this thesis a new approach was taken to the self-tuning problem. The usual ARMAX representation of a dynamical system was abandoned for a representation by an orthonormal series. The simple orthonormal Laguerre Ladder Network was first used for the derivation of these new self-tuning schemes. They proved to be robust, simple to use, not critical in initial parameter settings and capable of producing good control. Stability results have been obtained for the deterministic explicit self- tuning models and for some stochastic schemes. Deterministic, stochastic, single-input-singleoutput and multivariable schemes have been derived and were extensively tested on simulations. The proposed control schemes were designed using easily understood concepts, required minimal a-priori information, and proved capable of coping with some of the problems usually found in an industrial environment. They showed advantages of simplicity and flexibility while capable of achieving good control performance. Investigation has shown that the case is not at all restrictive and other orthonormal functions can also be used, although Laguerre functions proved to be a good choice for systems exhibiting time-delays and transient-signal responses. Some further examples were given based on a modified Laguerre set of functions and on a set of functions with complex exponential poles. Both these latter sets performed well and showed good identification capabilities.

An industrial application was presented. One of these newly developed self-tuning schemes was tested on a real industrial bleach plant. The new self-tuner behaved well 
and it provided good control to a non-linear $p H$ loop that exhibited a variable lengthy dead time. The results were well received by the mill's personnel because they meant a better loop performance to the bleach plant operators and process engineers along with substantial savings on the operational costs to the management. The new self-tuner proved to be robust, simple to use, capable of handling time delays and non-minimum phase plants, able to reject disturbances, and required minimal a priori information.

The developed schemes in this thesis are only good for stable plants. The numerical properties of the algorithms (implemented in single-precision), proved to be sound for a proper choice of $p$ and $T$ and for $N \leq 16$. The orthonormal sets that were reported in this dissertation provided good control for a certain class of systems but some other sets may be used or derived depending on the type of applications. Other control laws (e.g. state space methods) may be also tried out. An advantage of the techniques presented is that by adding an external white noise to the control input then the identification gives unbiased estimates even if the output disturbance is correlated noise. A problem that was found common with the ARMAX-based self-tuners related to the sometimes irregular initial transient period, i.e. before the identification converged. It was found that the value of the constant $\alpha$ of the reference trajectory could be adjusted accordingly to correct some of these problems.

Further research could be undertaken towards in-depth investigation of the theoretical properties of the algorithms and to present analytical results on their behaviour when compared with other existing schemes. From the application point of view a more rugged version of these control schemes can be developed as part of an expert-based system with main objectives being to simplify the application to a wide variety of processes, minimize processor load and memory requirements, handle bumpless transfer from manual to auto, and handle large process disturbances smoothly. 


\section{Bibliography}

[1] A kaike H. (1974), "A new look at the statistical model identification", IEEE Trans. Auto. Control, Vol. AC-19, p.716.

[2] Anderson B.D.O. and Moore J.B., (1971), "Linear Optimal Control", PrenticeHall, N.J.

[3] Åström, K.J., (1970), "Introduction to stochastic control theory", Academic Press, N.Y.

[4] Åström, K.J., (1972), "Process control in the pulp and paper industry. Notes from a course given in the Dept. of Auto. Control, Lund University, TFRT-3124.

[5] Åström, K.J., (1980a), "Design principles for self-tuning regulators", Intern. Symposium on Adaptive Systems, Ruhr University, Bochum, Germany.

[6] Åström, K.J., (1980b), "Self-tuning regulators", in Applications of Adaptive Control, ed. K.S. Narendra and R.V. Monopoly, Academic Press, N.Y.

[7] Åström, K.J., (1981), "Theory and applications of adaptive control", IFAC Congress, Kyoto, Japan.

[8] Åström, K.J., (1983), "Theory and applications of adaptive control: a survey", Automatica, vol 19, pp. 471-486.

[9] Åström, K.J. and B. Wittenmark, (1973), "On self-tuning regulators", Automatica, vol. 9, pp. 185-199. 
[10] Åström, K.J. and B. Wittenmark, (1980), "Self-Tuning controllers based on polezero placement", Proc. IEE, vol. 127, p.120.

[11] Athans M., (1971), "Linear-Quadratic-Gaussian control system design", IEEE Trans. Auto. Control, vol. AC-16, p.529.

[12] Axegard P. (1979), "Kinetics of Alkaline bleaching for the kraft CE sequence", Svensk Papperstidning, vol. 12, 361-367.

[13] Bartlett M.S. (1955), "Stochastic Processes". Cambridge University Press, Cambridge, England.

[14] Bendat J.S. and Piersol A.G. (1966), "Measurement and Analysis of Random Data", John Wiley, N.Y.

[15] Bengtsson G. and Egardt B., (1984), "Experiences with self-tuning control in the process industry", Proc. $9^{\text {th }}$ IFAC World Congress, Budapest, Hungary.

[16] Bierman G.J., (1977), "Factorization Methods for Discrete Sequential Estimation", Academic Press, N.Y.

[17] Bohn E.V. , (1982), "Estimation of continuous-time linear system parameters from periodic data", Automatica, vol.18, 2, pp.27-36.

[18] Borisson U. (1979), "Self-tuning regulators for a class of multivariable systems", Automatica, vol. 15, p.209.

[19] Box G.P. and Jenkins G.M. (1976), "Time Series Analysis Forecasting and Control", Holden-Day, CA.

[20] Chang R.Y. and Wang M.L., (1985), International Journal of Systems Science, vol. 16, p.1431. 
[21] Chow P.E.K. and Davies A.C. (1964), "The synthesis of Cyclic Code Generators", Electronic Engineering, Vol. April, pp.253-259.

[22] Chen C.F. and Hsiao C.H., (1975), Intern. Journal Systems Science, vol 6, p.833.

[23] Clarke, D.W. (1984), "Self-tuning control of nonminimum-phase systems", Automatica, vol. 20, pp. 501-517.

[24] Clarke, D.W. and P.J. Gawthrop, (1975), "A self-tuning controller", Proc. Inst. Elec. Eng., vol 122, pp.929-934.

[25] Clarke D.W., Hogdson A.J.F., Tuffs P.S. , (1983), "Offset problem and kincremental predictors in self-tuning control", Proceedings IEE, Vol. 130, Pt. D, 5, p.217.

[26] Clarke, D.W., C. Mohtadi and P.S. Tuffs, (1987), "Generalized predictive controlPart I. The basic algorithm", Automatica, vol.23, pp.137-148. Also as Oxford Univ. OUEL Techn. Report 1555, 1557/84.

[27] Corrington M.S. ,(1973), I.E.E.E. Trans. Circuit theory, vol. 20,p.470.

[28] Dahlin E.B. (1968), "Designing and Tuning Digital controllers", Part 1,2, Instrum. and Control Systems, vol. 7, p.125.

[29] Davison E.J., (1976), "The robust control of a servomechanism problem for linear time-invariant multivariable systems", IEEE Trans. Auto. Control, vol. AC-21, pp.25-34.

[30] Dugard L., Goodwin G.C., Xianya X. , (1984), "The role of the interacor matrix in multivariable stochastic systems", Automatica, vol.20, 5, pp.701-709. 
[31] Dumont G.A, (1988), "On the stability of the Rohrs example", personal communication.

[32] Dumont G.A, Martin-Sanchez J.M., Zervos C.C., (1988), "Comparison of an Autotuned PID Regulator and an Adaptive Control System on an Industrial Bleach Plant", to appear in Automatica Journal.

[33] Dumont G.A, Zervos C.C., Belangér P.R., (1984), "Automatic Tuning of Industrial PID Controllers", Technical Report PPR-505, Pulp and Paper Research Institute of Canada, Pointe-Claire,PQ.

[34] Dumont G.A, Zervos C.C.; Belangér P.R., (1985), "Automatic PID Controller Tuning: Industrial results", American Control Conference (ACC), Boston, MA.

[35] Dumont G.A. and Zervos C.C., (1986), "Adaptive Controllers based on Orthonormal Series Representation", $2^{\text {nd }}$ IFAC Workshop on Adaptive Systems in Control and Signal Processing, Lund, Sweden.

[36] Egardt B., (1980), IEEE Trans. Auto. Control, Vol. AC-25, p.693.

[37] Elliot H., (1982), "Direct adaptive pole placement with application to nonminimum phase systems", IEEE Trans. Auto. Control, vol. AC-27, p. 720.

[38] Elliott H. and Wolovich W.A. (1982), "A parameter adaptive control structure for linear multivariable systems", IEEE Trans. Auto. Control, vol. AC-27, p.340.

[39] Elliott H. and Wolovich W.A. (1984), "Parametrization issues in multivariable adaptive control", Automatica, vol.20, 5, pp.533-545.

[40] Eykhoff P., (1964), "Process Parameter Estimation", in Progress in Control Engineering, ed. MacMillan R.H., Academic Press, N.Y. 
[41] Feldbaum A.A. (1960), "Dual control theory", Automn and Remote Control, vol.21, p.874.

[42] Feldbaum A.A. (1961), ibid, vol.22, p.109.

[43] Francis B.A. and Wonham W. M. , (1975), "The internal model principle for linear multivariable regulators", Jrnl Applied Math. Optim., vol.2, pp. 170-194.

[44] Goodwin G.C. and Dugard L. (1983), "Stochastic adaptive control with known and unknown interactor matrices", IFAC Workshop on Adaptive Systems in control, San Francisco, CA.

[45] ' Goodwin G.C., McGinnis B.C., Wang J.C. (1982), "Model reference adaptive control for systems having non-square transfer functions", Proc. IEEE, CDC, Orlando, FLA.

[46] Goodwin G.C., Ramadge P.J., Caines P.E. (1982), "Discrete time stochastic adaptive control", SIAM, Jnl Control and Optimization, vol. 19, 6, p.829.

[47] Grimble M.J. (1984), "LQG self-tuning controllers", Automatica, vol.20, p.661.

[48] Grimble M.J. (1987), “ $H_{\infty}$ robust controller for self-tuning control applications. Part II : Self-tuners and Stability", Intern. Jnl of Control, vol. 46, No.5 , p.1819.

[49] Grimble M.J., Moir T.J. (1983), "Multivariable weighted minimum variance selftuning controllers", Preprints of IFAC Workshop on Adaptive Control and Signal Processing, San Francisco, CA.

[50] Goodwin G.C. and Sin K.S., (1984), "Pdaptive filtering prediction and control", Prentice-Hall Inc, N.J.

[51] Jury I.E.,(1958), "Sampled-data Control Systems", J. Wiley and sons, London. 
[52] Hooke R., Jeeves T.A., (1961), "Direct Search Solution of Numerical and Statistical Problems", J. Assoc: Comp. Mach., Vol. 8, pp. 212-229.

[53] Hoopes H.S., Hawk W.M, Lewis R.C. (1983), "A self-tuning controller", ISA Trans., vol.22:3, pp.48-58.

[54] Horng I.R. and Ho S.J., (1985), "Optimal control using discrete Laguerre polynomials", Intern. J. Control, vol.41, 6, pp.1613-1619.

[55] Horng I:R. and Ho S.J., (1986), "Discrete Walsh polynomials in the optimal control of linear digital systems", Intern: Journal of Control, vol. 43-2; pp.615-627.

[56] Hwang R.Y., Shih Y.P., (1983), "Combined methods for model reduction via discrete Laguerre polynomials", Intern. J. Control, vol.37, 3, pp.615-622.

[57] Ionescu T. and Monopoli R.V. (1977), "Discrete model reference adaptive control", Automatica, vol.13, p.156.

[58]. Jazwinski A.H. (1970), "Stochastic Process and Filtering Theory", Academic Press, N.Y.

[59] Kak S., (1974), I.E.E.E. Systems, Man, Cybernetics, vol. 4, p.399.

[60] Kalman, R.E., (1958), "Design of a self-optimizing control system", Trans. ASME, vol 80, pp. $468-478$.

[61] Kautz W.H., (1954), "Transient synthesis in the time domain", IRE Trans. Circuit Theory, September, pp.29-39.

[62] Keviczky L., Kumar K.S.P. (1981), "Multivariable self-tuning regulator with generalized cost-function", Intern. Inl of Control, vol.33, p.913. 
[63] · King R.E., Paraskevopoulos P.N. (1977), "Digital Laguerre filters", Circuit Theory and Appls., 5, 81-91.

[64] King R.E., Paraskevopoulos P.N. (1979), "Parametric identification of discretetime SISO systems", Intern. J. Control, vol.30, 6, pp.1023-1029.

[65] Koivo H.N. (1980), "A Mimo self-tuning controller", Automatica, vol.16, p.351.

[66] Lam K.P. (1980), "Implicit and Explicit self-tuning controllers", Research Rep. 1334/80, Engng Lab, University of Oxford.

[67] Landau I.D., (1974); "A survey of Model Reference Adaptive Techniques", Automatica, Vol. 10, p.356.

[68] Landau I.D., (1978), "Adaptive Control : The Model Reference Approach", Decker Publish. Co., N.Y.

[69] Lee Y.W., (1932), "Synthesis of Electrical networks by means of the Fourier transforms of Laguerre functions", J. Math. Phys., 11,pp.83-113.

[70] Lee T.T., Tsay S.C. and Horng I.R. (1986), "Shifted Jacobi series analysis of linear optimal control systems", Journal Franklin Institute, vol. 321-5, pp.289-298.

[71] Lee Y.W., (1960), "Statistical theory of Communication", J. Wiley, N.Y.

[72] Liu C.C. and Shih Y.P., (1984), Journal of Franklin Institute, vol. 317, p.373.

[73] Ljung L. (1977), "Analysis of recursive stochastic algorithms", IEEE Trans. Auto. Control, vol. AC-22, p.551.

[74] Ljung L. , (1987), "System Identification", Prentice Hall, N.J. 
[75] Ljung L. and Soderström T. , (1983), "Theory and Practice of Recursive Identification", The MIT Press, Cambridge, MA.

[76] MacFarlane A.G.J. (1979), "Complex-variable-design methods", in Modern approaches to control system design, ed N. Munro, P. Peregrinus Ltd.

[77] Makila P.M. (1984), "A self-tuning regulator based on optimal Output Feedback Theory", Automatica, vol.20, No.5, p.671.

[78] Martin-Sanchez J.M., (1976), "A new solution to adaptive control", Proc. IEEE, vol. 46 , pp. 1209-1218.

[79] Martin-Sanchez S.L., Shah S.L. (1984), "Multivariable adaptive predictive control of a Binary Distilation column", Automatica, September 1984.

[80] Mohtadi C., Shah S.L., Clarke D.W., (1987), "Generalized predictive control of multivariable systems", Proc. of the $5^{\text {th }}$ Yale Workshop on Appl. of Adaptive Systems, Yale Univ.

[81] Narendra K.S., Lin Y.H., Valavani L.S., (1980), "Stable Adaptive Controller Design", IEEE Trans. Auto. Control, Vol. AC-25, p. 230.

[82] Natarajan K. (1988), "On the Headbox flow dynamics", personal communication.

[83] Nease R.F., (1957), "Analysis and Design of Non-linear Sampled-data Control Systems", Technical Note 57-162, MIT, Boston, MA.

[84] Nurges U. and Jaksoo U., (1981), "Laguerre state equations of a multivariable discrete time system", IFAC 8th Triennial World Congress, Kyoto, Japan, pp.11531158. 
[85] Owens D.H. (1978), "Feedback and multivariable systems", IEE Control Engng series, No. 7.

[86] Parks P.C. (1966), "Lyapunov redesign of model reference adaptive control systems", IEEE Trans. Auto. Control, vol. AC-11, pp.362-67.

[87] Paul, R.P. (1981), "Robot manipulators - Mathematics, programming and control", MIT Press, Cambridge, MA.

[88] Payne, A.N. (1987), "Stability result with application to adaptive control", Int. J. Control, vol.46, pp. 249-261.

[89] Peterka V. (1970), "Adaptive digital regulation of noisy systems", IFAC Symposium on Ident. and Proc. Param. Estim., Prague.

[90] Peterka V. and Åström K.J. (1973), "Control of MIMO systems with unknown but constant parameters", Preprints of 3rd IFAC Symposium on Identification and process parameter Estimation, The Hague, Netherlands, p.535.

[91] Prager D.L. and Wellstead P.E. (1980), "MIMO pole-assignment self-tuning regulators", Proc. IEE, vol.128, Pt D, 1, p.9.

[92] Richalet J., A. Rault, J. Testud and J. Papon (1978), "Model predictive heuristic control: Applications to industrial processes". Automatica, vol. 14, pp. 413-428.

[93] Rissanen J. ,(1979), "Shortest data description and constistency of order estimates of an ARMA process", in Intern. Symposium on Systems Optimiz. and Analysis, ed. Bensoussan A., Lions J., Springer-Verlag, Berlin.

[94] Rohrs C.E. (1982), "Adaptive control in the presence of unmodeled dynamics", Ph.D thesis, Dept. of EE and Computer Science, MIT, LIDS, Report TH-1254. 
[95] Rohrs, C.E., Valavani L.S., Athans M., Stein G. (1982), "Robustness of adaptive control in the presence of unmodeled dymnamics", Proc. of the 21st IEEE CDC, 3, Orlando, FLA.

[96] Rohrs, C.E., L. Valavani, M. Athans and G. Stein (1985), "Robustness of continuous time adaptive control algorithms in presence of unmodeled dynamics", IEEE Trans. Automat. Contr., vol. 30, pp. 881-889.

[97] Rosenbrock H.H. (1979), "Inverse Nyquist array design method". in "Modern approaches to control system design", ed N. Munro, P. Peregrinus Ltd.

[98] Rouhani R. and Mehra R.K., (1982), "The Basic Theoretical Properties of Model Algorithmic Control", Automatica, vol. 18 ,No 4, pp.401-414.

[99] Samson, C. and J.J. Fuchs (1981), “ Discrete adaptive regulation of not-necessarily minimum-phase systems".Proc. IEE, vol. 128, Part D, pp. 102-108.

[100] Seborg D.L., Edgar T.F., Shah S.L., (1986), "Adaptive Control Strategies for process control: a survey", AIChE Journal, vol. 32-6, p.881.

[101] Singh R.P.,editor, (1979), "The Bleaching of Pulp". TAPPI monograph on Pulp Bleaching, Tappi Press, Atlanta, GA.

[102] Song H.K., Shah S.L., Fisher D.G., (1986), "A self-tuning robust controller", Automatica, vol.22, 5, pp.521-531.

[103] Stewart G.W.,(1973), "Introduction to Matrix Computations", Academic Press, N.Y.

[104] Strang G. (1976), Linear Algebra and its Applications, Academic Press, N.Y., pp. 223-224. 
[105] Strejc V. (1980), "Least Squares Parameter Estimation", Automatica, vol. 16, pp. 535-550.

[106] Toivonen H. (1984), "Multivariable adaptive control", Modelling, Identification and Control, vol. 5, pp.19-45.

[107] Trulsson Eva (1983), "Adaptive Control based on explicit criterion minimization", Ph.D. Dissertation S-581-83, Linkoping University, Sweden.

[108] Ydstie B.E., Kemna A.H., Liu L.K., (1987), "Robust Multivariable Predictive Control", Tech. Report, March 1987, Depart. Chem. Eng. , University of Mass. at Amherst, Amherst, MA.

[109] Waller, K.V. and Gustafsson, T.K, (1982), "Chemical reaction invariants in modeling and control of $\mathrm{pH} "$, Technical Report Å bo Akademi.

[110] Wellstead P.E., Edmunds J.M., Prager D., Zanker P. , (1979), "Self-tuning polezero assignment regulators", Intern. Journal of Control, vol. 30, p.1.

[111] Whitaker H.P., Yamrom J., Kezer A. (1958), "Design of Model-reference adaptive control systems for aircraft", Report R-164, Instrumentation Lab. MIT, Cambridge, Mass.

[112] Wiener N. (1956), "The theory of prediction". Modern Math. for Eng., Beckenbach (Ed), McGraw-Hill Co. N.Y. ,pp.183-184.

[113] Wolovich W. A. and Falb P.L. (1976), "Invariants and canonical forms under dynamic compensation", SIAM Jnl Control and Oplimization, vol.14, p.996.

[114] Ydstie, B.E. (1984), "Extended horizon adaptive control". IFAC 9th World Congress, Budapest, Hungary. 
[115] Young T.Y., W.H. Huggins (1961), "Representation of EKG by orthogonalized exponentials". IRE Inter. Conv. Rec., part 9, pp.145-153.

[116] Zervos C.C. (1984), "An off-line method for the optimal tuning of the three-term controller", M.Eng. thesis, McGill University, Montreal, PQ, Canada.

[117] Zervos C.C., P.R. Bélanger, G.A. Dumont (1985), "On PID controller tuning method using orthonormal series identification". IFAC Workshop on Adapt. Control Chem. Processes. Frankfurt, FRG. Also published in Automatica Journal, March, 1988.

[118] Zervos C.C. and Dumont G.A. (1988a), "Deterministic Adaptive Control based on Laguerre Series Representation", to appear in International Journal of Control. Also as PPRIC Tech. Report PGRL-391, Pulp and Paper Res. Inst. Canada, Pointe-Claire, PQ, Canada.

[119] Zervos C.C. and Dumont G.A. (1988b), "Laguerre Orthonormal Functions in Stochastic Self-Tuning Control", IFAC Workshop on Robust Adaptive Control, Newcastle, NSW, Australia.

[120] Zervos C.C. and Dumont G.A. (1988c), "Laguerre-based adaptive control of $\mathrm{pH}$ in an industrial bleach plant extraction stage", IFAC ADCHEM'88 symposium on Adaptive Control of Chem. proc., August, Lyngby, Denmark. 